\title{
Aproximações \\ de \\ Lógicas Modais
}

Guilherme de Souza Rabello

Dissertação apresentada ao Instituto de Matemática e Estatística da Universidade de São Paulo para obtenção do grau de Mestre em Ciências

Área de concentração: Matemática

Orientador: Prof. Dr. Marcelo Finger

São Paulo, Brasil

2005 


\section{Aproximações de \\ Lógicas Modais}

Guilherme de Souza Rabello

Dissertação apresentada ao Instituto de Matemática e Estatística da Universidade de São Paulo para obtenção do grau de Mestre em Ciências

Área de concentração: Matemática

BANCA EXAMINADORA

Prof. Dr. Marcelo Finger (IME/USP)

Prof. Dra. Renata Wassermann (IME/USP)

Prof. Dr. Walter Alexandre Carnielli (CLE/UNICAMP)

\section{SUPLENTES}

Prof. Dra. Maria Angela Weiss (IME/USP)

Prof. Dr. Mário Roberto Folhadela Benevides (COPPE/UFRJ) 
A meus pais

e

a Maria Angela Weiss 


\section{Agradecimentos}

Para todos os que me ajudaram de alguma forma na vida, os meus mais sinceros agradecimentos.

Acima de todos, agradeço a meus pais, Ivonete de Souza Rabello e Getúlio Daré Rabello; a eles devo minha vida, minha educação e toda possibilidade de fazer este trabalho - jamais poderei retribuir-lhes de forma adequada.

Uma fonte eterna de inspiração, o poeta norte-americano Edgar Allan Poe encheu minha alma de adoração à beleza; a ele devo toda minha cultura.

Lado a lado com Poe está o filósofo polonês Jan Lukasiewicz, responsável por minha decisão de trabalhar em Lógica Matemática; meu débito por seus escritos é impagável.

Agradeço a meu orientador, Marcelo Finger, pelo entusiasmo contagiante e pela excelente condução de meu trabalho; ele acreditou em mim e me deu a mão no momento mais difícil de todo meu mestrado.

Nos países mais diversos, alguns pesquisadores foram de auxílio inestimável pelo bom completamento deste texto; agradeço a Hector Levesque, de Toronto (Canadá), a Per Hasle, de Aalborg (Dinamarca), e a Marcelo D'Agostino, de Ferrara (Itália) - em particular, à secretária de Hector Levesque, Elaine Coutsoyannopoulos, que teve a imensa paciência de fazer uma cópia eletrônica de dois artigos de Levesque, fundamentais à minha pesquisa.

Os meus amigos são poucos mas valiosos; agradeço a eles pelos bons momentos juntos, que me fizeram relaxar e suportar todas as pressões que naturalmente decorrem de um empreendimento desses - são eles Lécio de Oliveira Arantes e David Augaitis Fogaça.

Gostaria de agradecer a meus dois irmãos, Lúcia e Francisco de Souza Rabello; ainda que nossos caminhos na vida estejam sendo bastante diferentes, com eles passei a maior parte de minha vida e, assim espero, com eles manterei o laço de fraternidade para o resto dela.

Uma pessoa cuja pesquisa certamente serviu de inspiração foi Renata Wassermann; a ela devo também ter conhecido meu orientador, quando cheguei "com uma mão na frente e outra atrás" buscando alguém no IME que pudesse me orientar na área de lógicas não clássicas.

Entre os professores que mais me estimularam no estudo de lógica em geral, o nome de Stavros Christodoulou, meu primeiro professor de Lógica Matemática, sobressai; grandes são as saudades que sinto dele.

Imediatamente após fazer o curso com o Stavros, conheci Piotr Bolesław Koszmider, professor e pesquisador de Lógica e Teoria dos Conjuntos; com ele aprendi mais matemática do que jamais sonhara aprender, e seu nome não poderia deixar de figurar nestes agradecimentos.

Relacionada a tudo também está a professora Zara Issa Abud, uma das pessoas mais maravilhosas que conheço; sempre empenhada em ajudar, ela me guiou nos primeiros e assustados passos que dei nos complicados estudos que compõem a Matemática. 
Antes de todos esses professores, agradeço a meu grande professor de Física do último ano do Ensino Médio, Sílvio Pélico; naquele ano difícil da minha vida (1996) ele foi o único a acreditar em mim e, principalmente, a me apoiar na decisão de fazer uma faculdade numa área de Ciências Fundamentais.

Devo à Comissão de Admissão e Bolsas e a seus membros, em particular a Daniel Victor Tausk, a concessão da importante bolsa institucional de mestrado durante o último ano e meio, sem a qual eu jamais teria completado este trabalho; o Daniel, em especial, deu-me esclarecimentos importantes no momento em que eu precisava.

Eu também quero agradecer ao Mário Roberto Folhadela Benevides, que aceitou o convite de participar da banca examinadora da minha defesa de mestrado na última hora e mesmo assim leu com atenção e cuidado meu trabalho, fazendo correções muito importantes.

Além de tantos indivíduos relacionados diretamente ao meu trabalho, há aqueles que foram importantes na minha vida de outra forma e que, por essa razão, merecem ser lembrados; meu bisavô José Baptista Daré, falecido há quase 10 anos (dezembro de 1995) foi uma pessoa fundamental em minha vida e ainda lamento sua perda - que foi, para mim, algo inesperado, pois eu o julgava imortal.

Lamento não menos a perda da minha tia-avó Geralda Alves de Souza, uma pessoa que sempre gostou de mim e estimulou minhas leituras; sinto falta de sua conversa e de sua presença em minha vida.

Meu avô, Joaquim Alves de Souza, é uma pessoa por quem nutro profunda admiração e afeto; muito aprendi com ele, e ainda espero muito aprender.

Eu também preciso citar minha amada avó, Deolinda Daré Rabello, uma das pessoas mais impressionantes que jamais conheci, pela atividade, pela independência e principalmente pela vida que dela emana; ela é um exemplo para mim, pois após tantas vicissitudes ainda mantém a serenidade.

Infinita é minha adoração por uma pessoa que eu nunca poderia deixar de citar aqui: a minha namorada, Thaís Lopes Costa. A ela devo conforto, carinho e amor, que muito contribuíram para minha paz de espírito e minha sanidade no último ano do mestrado - Thaís, eu amo você!

Depois de todas essas pessoas, mas não menos importante (pelo contrário!), cito a minha grande amiga e professora Maria Angela Weiss, que foi fundamental em todos os estágios da redação deste texto; a ela devo o apoio no momento de dificuldade por que passei durante o mestrado e também o estímulo indispensável - dedico a ela qualquer virtude que este trabalho tenha (e cujos defeitos se devem unicamente a mim).

Agradeço, por fim, ao Conselho Nacional de Desenvolvimento Científico e Tecnológico - CNPq, entidade governamental brasileira promotora do desenvolvimento científico e tecnológico, de quem recebi apoio financeiro. Espero, com meu trabalho, ter feito jus à confiança dessa ilustre instituição. 
Oinos. - But in this existence, I dreamed that I should be at once cognizant of all things, and thus at once happy in being cognizant of all.

Agathos. - Ah, not in knowledge is happiness, but in the aquisition of knowledge! In for ever knowing, we are for ever blessed; but to know all were the curse of a fiend.

- Edgar Allan Poe, The power of words, in [Poe93] 


\section{Resumo}

Este texto tem por objetivo estudar aproximações de lógicas, um procedimento que tem interesse tanto filosófico quanto computacional. No primeiro capítulo, é apresentado o conceito geral de aproximações. Nos três capítulos seguintes, diferentes abordagens encontradas na literatura são discutidas. Primeiro, o procedimento de Cadoli-Schaerf [SC95], que foi um dos primeiros e ainda é um dos mais importantes métodos de aproximações de lógicas, tendo servido de base para os outros dois aqui analisados. Cadoli e Schaerf trabalham com sentenças no formato normal clausal e normal negativo. $\mathrm{O}$ trabalho de Cadoli-Schaerf apresenta procedimentos de aproximações para lógicas proposicionais clássicas, formaliza esses procedimentos em uma lógica modal do conhecimento e os estende para modais. O segundo procedimento, de Fabio Massacci [Mas98a, Mas98b], estende Cadoli-Schaerf para sentenças proposicionais quaisquer e para sentenças modais, todas assinaladas; além disso, apresenta uma teoria da prova a partir de tableaux semânticos, também utilizando sentenças assinaladas. $O$ terceiro procedimento, devido a Finger e Wassermann [FW01, FW02], generaliza Cadoli-Schaerf para sentenças proposicionais quaisquer não-assinaladas, empregando sentenças assinaladas apenas nos tableaux de prova. Nos dois capítulos que se seguem, é apresentada uma extensão de Finger-Wassermann para lógicas modais - com semântica e teoria da prova - e formaliza-se esse procedimento em uma lógica modal do conhecimento. Na conclusão, analisam-se os resultados obtidos e discutem-se possíveis direções para trabalhos futuros. 


\begin{abstract}
This text has as objective the study of approximations of logics, a procedure that has philosophical as well as computational interest. In the first chapter, the general concept of approximations is presented. In the next three chapters, different approaches found in the literature are discussed. First, the procedure of Cadoli-Schaerf [SC95], one of the first and still one of the most important methods of approximations of logics, having served as the basis for the other two here analised. Cadoli and Schaerf work with sentences in normal clausal and negative normal formats. The work of Cadoli-Schaerf presents procedures of approximations for classical propositional logics, formalizes these procedures in a modal logic of knowledge and extends them to modal logics. The second procedure, of Fabio Massacci [Mas98a, Mas98b], extends Cadoli-Schaerf to any propositional sentences and also to modal sentences, always signed; besides, he presents a proof theory using semantic tableaux, also using signed sentences. The third procedure, by Finger and Wassermann [FW01, FW02], generalizes Cadoli-Schaerf to any non signed propositional sentences, using signed sentences only in the proof tableaux. In the two chapters that follow, an extension of Finger-Wassermann to modal logics is presented - with semantics and proof theory - and the procedure is formalized in a modal logic of knowledge. In the conclusion, the results achieved are analised and possible directions for future research are discussed.
\end{abstract}




\section{Lista de Figuras}

2.1 Um exemplo de aplicação dos Tableaux de Smullyan . . . . . . 17

2.2 Invalidação do princípio modal $4 \ldots \ldots . \ldots . . . \ldots 25$

2.3 Um exemplo de aplicação dos SST . . . . . . . . . . . . . . 30

4.1 Um exemplo de aplicação dos Tableaux Proposicionais Aproximados ..................... 50

5.1 Um exemplo de aplicação dos $\mathrm{KES}_{3}$-tableaux $\ldots . . . . . . .60$

6.1 Prova de $K$ por $\left\langle S_{3}, n\right\rangle$-SST . . . . . . . . . . . . . 69

6.2 Prova de $\diamond(p \supset p)$ na lógica modal $\mathbf{D}$ por $\left\langle S_{3}, n\right\rangle-\mathrm{SST} \ldots \ldots . .69$

6.3 Prova de $\diamond(p \supset \square p)$ na lógica modal T por $\left\langle S_{3}, n\right\rangle$-SST $\ldots \ldots .70$

6.4 Prova de $\square p \supset \square \square \square p$ na lógica modal S5 por $\left\langle S_{3}, n\right\rangle$-SST . . . 70

6.5 Prova de $\square(p \supset \neg \neg p)$ por $\left\langle S_{3}, n\right\rangle$-SST . . . . . . . . . . . . 71

6.6 Prova de $K$ por $\left\langle S_{3}, n\right\rangle^{\prime}$-SST . . . . . . . . . . . . . . 81

6.7 Prova de $\square p \supset \square \square p$ na lógica modal S4 por $\left\langle S_{3}, n\right\rangle^{\prime}$-SST . . . 82

6.8 Prova de $\square p \supset \square \square \square p$ na lógica modal S5 por $\left\langle S_{3}, n\right\rangle^{\prime}$-SST . . . 83 


\section{Lista de Tabelas}

2.1 Notação unificadora de Smullyan . . . . . . . . . . . . . . . 16

2.2 Regras de Smullyan para Lógica Clássica . . . . . . . . . . . . 16

2.3 Regras KE para Lógica Clássica . . . . . . . . . . . . . . . . . . 19

2.4 Axiomas modais e respectivas sentenças . . . . . . . . . . 21

2.5 Caracterização dos axiomas modais . . . . . . . . . . . 24

2.6 Lógicas modais e respectivas caracterizações . . . . . . . . . 25

2.7 Notação unificadora de Fitting . . . . . . . . . . . . . . . . 26

2.8 Regras proposicionais para SST . . . . . . . . . . . . . . 27

2.9 Regras modais para SST válidas em todas as lógicas . . . . . . . 28

2.10 Regras modais para SST que caracterizam lógicas . . . . . . . . 28

2.11 Lógicas modais e respectivas regras SST . . . . . . . . . . . 29

4.1 Significado superficial das sentenças proposicionais . . . . . . 48

4.2 Significado superficial das sentenças modais . . . . . . . . . . . 54

6.1 Lógicas modais e condições sobre a relação sintática $\triangleright \quad \ldots \ldots 75$ 


\section{Sumário}

1 Introdução 1

1.1 O Sistema $\mathbf{L}$ de Levesque . . . . . . . . . . . . . . 1

1.2 Compilação de Conhecimento de Selman e Kautz . . . . . . . 7

1.3 O Conceito de Aproximações de Lógicas . . . . . . . . . . 8

1.4 Objetivos ...................... 9

1.5 Estrutura da dissertação . . . . . . . . . . . . . . 9

2 Preliminares 11

2.1 Lógica Proposicional Clássica . . . . . . . . . . . . . . . 11

2.1.1 Notação, Sintaxe e Semântica . . . . . . . . . . . . . . . 11

2.1.2 Tableaux de Prova . . . . . . . . . . . . . . . . . 14

2.2 Lógicas Proposicionais Modais . . . . . . . . . . . . 20

2.2 .1 Notação, Sintaxe e Semântica . . . . . . . . . . . . 20

2.2 .2 Tableaux de Prova . . . . . . . . . . . . 25

3 O Método de Cadoli-Schaerf 32

3.1 Semântica e Principais Propriedades . . . . . . . . . . . . 33

3.2 Modalidades $\square_{S}^{1}$ e $\square_{S}^{3} \ldots \ldots \ldots \ldots \ldots \ldots \ldots \ldots$

3.3 Aplicação a Lógicas Modais . . . . . . . . . . . . . . . 40

4 O Método de Massacci 46

4.1 Tableaux Proposicionais Aproximados . . . . . . . . . . 47

4.2 Tableaux Modais Aproximados . . . . . . . . . . . . . 51

5 O Método de Finger-Wassermann 56

5.1 Semântica e Principais Propriedades . . . . . . . . . . 56

5.2 Tableaux de Prova . . . . . . . . . . . . . . . . 59

6 Finger-Wassermann para Lógicas Modais 62

6.1 Semântica e Principais Propriedades . . . . . . . . . . . . 62

6.2 Tableaux de Prova . . . . . . . . . . . . . . . . . 68

6.3 Semântica e Teoria de Prova Alternativos . . . . . . . . . . 77

6.4 Uma Semântica mais Geral . . . . . . . . . . . . . . . . . 84

7 Lógica do Conhecimento de Finger-Wassermann $\quad 87$ 
8 Conclusões

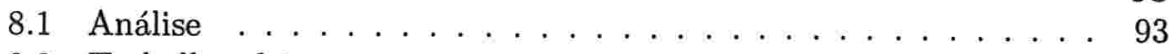

8.2 Trabalhos futuros . . . . . . . . . . . . . . 96 


\section{Capítulo 1}

\section{Introdução}

George Boole, matemático inglês do século XIX e considerado o fundador da lógica moderna, definia a lógica como a ciência das "leis fundamentais daquelas operações mentais pelas quais o raciocínio é realizado" [Boo54]. Muito do desenvolvimento posterior enfatizou as relações entre lógica e fundamentos da matemática, deixando de lado sua utilização como modelo para um agente pensante. Mas, com o desenvolvimento da Inteligência Artificial (Artificial Intelligence, AI), que por definição da American Association for Artificial Intelligence (AAAI) é a "compreensão científica dos mecanismos subjacentes ao pensamento e ao comportamento inteligente e sua incorporação em máquinas" ${ }^{1}$, a utilização de lógica como base de formalização de problemas de representação do conhecimento (Knowledge Representation, KR) mostrou-se, em diversos casos, bemsucedida, retomando, de certa maneira, o ideal de Boole.

Este trabalho traça a evolução de um método para representação do conhecimento utilizando lógica. Esse método controla a quantidade de conhecimento disponível ao agente pensante e a quantidade de inferências de que ele é capaz; além disso, é um método com semântica bem determinada e que pode ser incrementado (se houver mais tempo e espaço disponíveis em um banco de dados, por exemplo), tendo portanto aplicações na ciência da computação.

\subsection{O Sistema $L$ de Levesque}

Em seu artigo "A logic of implicit and explicit belief" [Lev84], Hector Levesque observa que um problema recorrente na modelagem de crenças ou conhecimento ${ }^{2}$ é o que foi chamado por Hintikka em [Hin75] de onisciência lógica. De maneira resumida, a onisciência lógica de um agente significa que ele consegue obter todas as conseqüências lógicas de suas crenças. "É simplesmente construído na lógica" - afirma Levesque no artigo supracitado - "que, se $\phi$ é acreditada

\footnotetext{
${ }^{1}$ Definição extraída da página da AAAI, http://ww . aaai.org.

${ }^{2}$ Levesque prefere utilizar a expressão "crenças" para aquilo que é representado nos bancos de dados, já que em geral não se requer que isso seja verdadeiro.
} 
e $\phi$ logicamente implica em $\psi$, então $\psi$ é acreditada também". Um agente real, limitado, não consegue chegar a todas as conclusões de suas crenças, e a abordagem a partir da semântica de mundos possíveis é "não-intuitiva na melhor das hipóteses e completamente não-realística na pior" [Lev84].

Levesque sugere uma possível solução para o problema, apontando a diferença entre o que um agente de fato acredita que ocorra - a chamada crença explícita, representada pelo operador $B$ - e o que segue daquilo que ele acredita - a chamada crença implícita, representada pelo operador $L$. A modelagem da crença implícita, originada por Hintikka em [Hin62], é feita pela semântica de mundos possíveis de Kripke para a lógica modal $\mathrm{S5}^{3}$, e contém dentro de si o problema da onisciência lógica. $\mathrm{Na}$ linguagem modal, a onisciência lógica é expressa por $\square p \wedge \square(p \supset q) \supset \square q$, que é equivalente ao princípio $K-$ aqui, a sentença $\square \phi$ pode ser lida como " $\phi$ é acreditada ou válida". Para as crenças explícitas, Levesque generaliza a semântica de Kripke para uma de situaçôes. Em outras palavras, os mundos possíveis de Kripke são uma classe especial de situações de Levesque. Matematicamente, a generalização é efetivada da seguinte maneira. Enquanto os mundos possíveis (clássicos) dão uma valoração total no conjunto dos literais (ou seja, dos átomos ou das negações de átomos - um átomo e sua negação são literais conjugados), as situações dão uma valoração parcial nesse conjunto. Ser parcial, nesse contexto, significa que nem todo elemento do domínio é necessariamente utilizado: podem haver literais sem valoração; a valoração é total justamente quando todo elemento do domínio for utilizado. Intuitivamente, o fato de que cada situação corresponde a uma valoração parcial significa que o agente pensante não associa um valor verdade a todas as proposições; um agente dotado de recursos finitos nem poderia fazer isso. Na teoria da prova, o efeito dessas concepções é um enfraquecimento das regras de inferência para o operador $B$. Em particular, modus ponens não vale em geral.

Algumas situações que correspondem às crenças explícitas do agente são selecionadas e colocadas em um conjunto $\mathcal{B}$, chamado conjunto de situações acreditadas. Esse conjunto representa o conhecimento explícito do agente pensante. A cada situação, o agente pensante associará uma valoração apenas às proposições que representem um conhecimento importante ou relevante para si.

A seguir, será apresentado o sistema $\mathbf{L}$ de Levesque de maneira adaptada.

Uma notação conveniente ao texto: se $X$ é um conjunto de átomos, $X^{\circ}$ é o conjunto de todos os literais associados a esses átomos. Seja $A$ o conjunto dos átomos da linguagem. Um modelo para $\mathbf{L}$ é uma tripla $\mathcal{M}=\langle$ Sit, $\mathcal{B}, V\rangle$, em que Sit é um conjunto não vazio de situações, $\mathcal{B} \subseteq$ Sit é o conjunto de situações acreditadas e $V:$ Sit $\rightarrow\left(A^{\circ} \rightarrow\{0,1\}\right)$ é uma função que associa a cada situação uma valoração parcial no conjunto $A^{\circ}$ dos literais.

$\mathrm{O}$ conjunto dos mundos possíveis $\mathcal{W}(s)$ em relação à situação $s$ é o conjunto de todos $s^{\prime} \in$ Sit tal que, para todo $p \in A$,

a) $V\left(s^{\prime}\right)$ é uma valoração total

\footnotetext{
${ }^{3}$ Uma apresentação dos axiomas e regras de inferência desse e de outros sistemas modais é dada na Seção 2.2 .
} 
$\left.b_{1}\right)$ se $V\left(s^{\prime}\right)(p)=1$, então $V\left(s^{\prime}\right)(\neg p)=0$

$\left.b_{2}\right)$ se $V\left(s^{\prime}\right)(p)=0$, então $V\left(s^{\prime}\right)(\neg p)=1$

$\left.c_{1}\right)$ se $V(s)(p)=1$, então $V\left(s^{\prime}\right)(p)=1$

$\left.c_{2}\right)$ se $V(s)(p)=0$, então $V\left(s^{\prime}\right)(p)=0$

Assim, os mundos possíveis em relação a uma situação $s$ são as situações que mapeiam cada par de literais conjugados em valores opostos (sendo portanto associadas a valorações totais), e que concordam com $s$ nos átomos. Dado um subconjunto Sit $^{\prime} \subseteq S i t$, define-se $\mathcal{W}\left(\right.$ Sit $\left.t^{\prime}\right)$ como a união de todos os $\mathcal{W}(s)$ para $s \in$ Sit'. $^{\prime}$.

A semântica de $\mathbf{L}$ é uma relação $\models \subseteq$ Sit $\times \mathbf{L}$ dada pelas seguintes regras (em que uma sentença proposicional é uma sentença que utiliza apenas os operadores clássicos):

L1. $\mathcal{M}, s \models p$ sse $V(s)(p)=1$

$\mathcal{M}, s \models \neg p$ sse $V(s)(\neg p)=1$

L2. $\mathcal{M}, s \models \phi \vee \psi$ sse $\mathcal{M}, s \models \phi$ ou $\mathcal{M}, s \models \psi$

$\mathcal{M}, s \models \neg(\phi \vee \psi)$ sse $\mathcal{M}, s \models \neg \phi$ e $\mathcal{M}, s \models \neg \psi$

L3. $\mathcal{M}, s \models \phi \wedge \psi$ sse $\mathcal{M}, s \models \phi$ e $\mathcal{M}, s \models \psi$

$\mathcal{M}, s \models \neg(\phi \wedge \psi)$ sse $\mathcal{M}, s \models \neg \phi$ ou $\mathcal{M}, s \models \neg \psi$

L4. $\mathcal{M}, s \models \neg \neg \phi$ sse $\mathcal{M}, s \models \phi$,

L5. $\mathcal{M}, s \models B \phi$ sse $\phi$ é proposicional e, para todo $s^{\prime} \in \mathcal{B}, \mathcal{M}, s^{\prime} \models \phi$ $\mathcal{M}, s \models \neg B \phi$ sse $\mathcal{M}, s \not \models B \phi$

L6. $\mathcal{M}, s \models L \phi$ sse $\phi$ é proposicional e, para todo $s^{\prime} \in \mathcal{W}(\mathcal{B}), \mathcal{M}, s^{\prime} \models \phi$

$\mathcal{M}, s \models \neg L \phi$ sse $\mathcal{M}, s \not \models L \phi$

Essa semântica se assemelha à semântica de S5, embora não seja equivalente (não existe, por exemplo, um conjunto $\mathcal{B}$ em S5). Isso porque Levesque procurou refinar o trabalho de Hintikka, que associou S5 à Lógica do Conhecimento. Observa-se que a semântica é paraconsistente, pois podem haver situações em que tanto $\phi$ como $\neg \phi$ são verdadeiras. Nota-se também que os operadores $B$ e $L$ só se aplicam a sentenças proposicionais (ou seja, não há interação desses operadores). A sentença $\phi \supset \psi$ é equivalente $a \neg \phi \vee \psi$.

Intuitivamente, $B \phi$ significa que a sentença $\phi$ é acreditada explicitamente. Uma vez que a condição de verdade para $B \phi$ depende unicamente de situações no conjunto $\mathcal{B}$, e situações são mais gerais que mundos, a crença explícita em uma sentença é algo como uma "leitura rasa", que passa por cima de eventuais contradições que possam acontecer. A crença implícita $L \phi$ depende não das situações no conjunto $\mathcal{B}$, mas dos mundos possíveis em relação às situações $\mathcal{B}$ ou seja, é algo mais restrito, que não passa por cima de contradições eventuais. Uma das conseqüências é que toda crença explícita é também implícita, ou seja, 
$B \phi \supset L \phi$ : se uma sentença é acreditada explicitamente, ela é verdadeira para todas as situações em $\mathcal{B}$ e, em particular, para todos os mundos possíveis em relação às situações em $\mathcal{B}$.

Uma sentença $\phi$ é válida, $\models \phi$, se para todo modelo $\mathcal{M}$ e todo mundo possivel $w$ - e não em toda situação - ocorre $\mathcal{M}, w \models \phi$. Uma sentença $\phi$ é satisfativel se existe um modelo $\mathcal{M}$ e um mundo possível $w$ tal que $\mathcal{M}, w \models \phi$. Uma sentença $\phi$ implica logicamente outra sentença $\psi, \phi \models \psi$, se todo modelo e todo mundo possível que satisfaz $\phi$ satisfizer também $\psi$. Para o operador $L$, são satisfeitas trivialmente a regra da necessitação $(\models \phi$ implica $\models L \phi)$ e onisciência lógica $(\vDash L \phi \wedge L(\phi \supset \psi) \supset L \psi)$. É com o operador $B$ que começam a ocorrer coisas interessantes. Como Levesque afirma, os seguintes conjuntos são satisfativeis:

1. $\{B p, B(p \supset q), \neg B q\}$, o que mostra que para crenças explícitas não vale modus ponens. Isso ocorre, por exemplo, se:

- $V(s)(p)=V(s)(\neg p)=1$

$$
V(s)(q)=0
$$

- Sit $=\mathcal{B}=\{s\}$

Para o modelo $\mathcal{M}=\langle$ Sit, $\mathcal{B}, V\rangle$, tem-se $\mathcal{M}, s \models p$ e $\mathcal{M}, s \models \neg p$ - essa última relação implica que $\mathcal{M}, s \models p \supset q-$, $\operatorname{mas} \mathcal{M}, s \not \models q$. Como $s \in \mathcal{B}$, a primeira e terceira relações implicam que $\mathcal{M}, s \models B p$ e $\mathcal{M}, s \models B(p \supset q)$, respectivamente; porém $\mathcal{M}, s \not \models q$ implica que $\mathcal{M}, s \not \models B q$ e, portanto, que $\mathcal{M}, s \models \neg B q$. Como $s$ é a única situação desse modelo, o conjunto $\{B p, B(p \supset q), \neg B q\}$ é satisfatível.

2. $\{\neg B(p \vee \neg p)\}$, o que mostra que sentenças válidas não precisam ser explicitamente acreditadas. Isso ocorre se:

- $V=\emptyset$

- Sit $=\mathcal{B}=\{s\}$

Para o modelo $\mathcal{M}=\langle$ Sit, $\mathcal{B}, V\rangle$, tem-se $\mathcal{M}, s \not \forall p$ e $\mathcal{M}, s \not \forall \neg p-\mathrm{e}$, portanto, $\mathcal{M}, s \not \models p \vee \neg p$. Dessa forma, não ocorre $\mathcal{M}, s \models B(p \vee \neg p)$, ou seja, ocorre $\mathcal{M}, s \models \neg B(p \vee \neg p)$. Como $s$ é a única situação desse modelo, o conjunto $\{\neg B(p \vee \neg p)\}$ é satisfatível.

3. $\{B p, \neg B(p \wedge(q \vee \neg q))\}$, o que mostra que sentenças logicamente equivalentes a sentenças explicitamente acreditadas não precisam ser explicitamente acreditadas. Isso ocorre se:

- $V(s)(p)=1$ mas $\neg p, q$ e $\neg q$ não são associados a nenhuma valoração

- Sit $=\mathcal{B}=\{s\}$

Para o modelo $\mathcal{M}=\langle$ Sit, $\mathcal{B}, V\rangle$, tem-se $\mathcal{M}, s \models p$ (e, como $s \in \mathcal{B}$, isso implica que $\mathcal{M}, s \models B p$ ), mas não ocorre nem $\mathcal{M}, s \models q$ nem $\mathcal{M}, s \models \neg q$ - e, portanto, $\mathcal{M}, s \not \models q \vee \neg q$. Dessa forma, não ocorre $\mathcal{M}, s \models p \wedge(q \vee \neg q)$ e, com isso, não pode ocorrer $\mathcal{M}, s \models B(p \wedge(q \vee \neg q))$. Assim, $\mathcal{M}, s \models$ 
$\neg B(p \wedge(q \vee \neg q))$. Como $s$ é a única situação desse modelo, o conjunto $\{B p, \neg B(p \wedge(q \vee \neg q))\}$ é satisfatível.

4. $\{B p, B \neg p, \neg B q\}$, o que mostra que crenças explícitas podem ser inconsistentes sem que toda sentença seja acreditada - ou seja, crenças explícitas podem ser paraconsistentes. Isso ocorre se:

- $V(s)(p)=V(s)(\neg p)=1$ $V(s)(q)=0$

- Sit $=\mathcal{B}=\{s\}$

Para o modelo $\mathcal{M}=\langle$ Sit $, \mathcal{B}, V\rangle$, tem-se $\mathcal{M}, s \models p$ e $\mathcal{M}, s \models \neg p$, mas $\mathcal{M}, s \not \neq q$. Como $s \in \mathcal{B}$, as duas primeiras relações implicam, respectivamente, que $\mathcal{M}, s \models B p$ e $\mathcal{M}, s \models B \neg p$, mas a terceira não pode implicar que $\mathcal{M}, s \models B q$, implicando, assim, que $\mathcal{M}, s \models \neg B q$. Como $s$ é a única situação desse modelo, o conjunto $\{B p, B \neg p, \neg B q\}$ é satisfatível.

Levesque apresenta uma teoria da prova para essa semântica, e mostra que a teoria generaliza a Lógica da Relevância de Anderson e Belnap [AB75] — ou seja, a Lógica da Relevância é um caso particular de L. A Lógica da Relevância lida com uma relação de inferência que tem por intuição o fato de que o antecedente de uma implicação deve ser relevante para o conseqüente. Levesque mostra, em [Lev84], que $\models B \phi \supset B \psi$ sse $\phi$ é relevante para $\psi$. Ele também prova a correção e completude de sua teoria. Para esses detalhes, refere-se ao artigo já citado, [Lev84]. Aqui, só se observa que nessa teoria da prova valem todas as regras da Lógica Clássica e que haverá um axioma para onisciência lógica do operador $L$, ou seja, $(L \phi \wedge L(\phi \supset \psi)) \supset L \psi$. Isso, junto com as regras da Lógica Clássica, implica que modus ponens vale para crenças implícitas. Tal axioma não existe para o operador $B$, o que implica que modus ponens não vale para crenças explícitas, como havia sido dito anteriormente.

Em [Lev89], Levesque adiciona uma restrição à função $V$, para que toda tautologia na forma normal conjuntiva, CNF (isto é, uma conjunção de cláusulas; cláusulas são disjunções de literais), possa ser explicitamente acreditada. Em primeiro lugar, ele passa a exigir que toda situação seja associada a uma valoração total. A restrição também obriga que, para cada situação $s \in$ Sit, pelo menos um dos pares de literais seja levado no valor 1 , ou seja, dados $s \in \mathcal{S}$ e $p \in L$ quaisquer, sempre ocorre ou $V(s)(p)=1$ ou $V(s)(\neg p)=1$ (e possivelmente $V(s)(p)=V(s)(\neg p)=1)$. Com essas restrições, os exemplos 2 e 3 acima não mais se aplicam, mas os exemplos 1 e 4 continuam valendo. É essa forma do sistema de Levesque que Cadoli e Schaerf generalizaram (ver Capítulo 3).

Definição 1.1 Uma valoração total que, de cada par de literais conjugados, leva pelo menos um para o valor 1 é chamada de 3-interpretação. Uma valoração clássica, que leva um par de literais conjugados para valores opostos, é chamada de 2-interpretação.

O nome 3-interpretação vem do fato que, para cada par de literais conjugados, há três possibilidades: $V(s)(p)=1$ e $V(s)(\neg p)=0, V(s)(p)=0$ e $V(s)(\neg p)=1$ 
ou $V(s)(p)=1$ e $V(s)(\neg p)=1$. Assim, a função $V(s)$, para cada $s$, é uma 3 -interpretação.

Com a restrição sobre a função $V$ dada acima, é possível modelar a lógica das crenças explícitas sem o operador $B$, utilizando uma relação $\models^{3}$ definida da seguinte maneira.

Definição 1.2 Seja $\Gamma$ um conjunto de sentenças. Define-se $\Gamma \models^{3} \psi$ sse toda 3-interpretação que satisfaz todas as sentenças em $\Gamma$ satisfizer também $\psi$.

Observa-se que $\models^{3} \phi$ significa que toda 3-interpretação satisfaz $\phi$, ou seja, que $\emptyset \models{ }^{3} \phi$. Denota-se $\{\phi\} \models \psi$, de maneira mais simples, por $\phi \models \psi$.

Dada uma sentença $\phi$ em CNF, se for considerado que $\neg \phi$ é sua negação respectiva em CNF, não pode acontecer simultaneamente que $\phi \models^{3} \psi$ e $\phi \models^{3}$ $\neg \psi$. Isso porque $\phi \models^{3} \psi$ significa que toda 3 -interpretação que satisfaz $\phi$ satisfaz também $\psi$-em particular, para as 3-interpretações que são 2-interpretações também. Para essas últimas, com comportamento clássico, não pode ocorrer $\phi \models^{3} \neg \psi$. Com esse mesmo argumento é possível notar que, se $\phi \models^{3} \psi$, então $\phi \models \psi$.

A ligação de $\models^{3}$ com o operador $B$ (definido por Levesque com a restrição posterior em seu artigo [Lev89]) é a seguinte:

Teorema $1.3 \phi \models^{3} \psi$ sse $\models B \phi \supset B \psi$.

Dem: $\quad(\Rightarrow)$ Primeiro, suponha que $\phi \models^{3} \psi$ mas $\not \models B \phi \supset B \psi$. Então, existe um modelo $\mathcal{M}$ e uma situação $s$ tais que $\mathcal{M}, s \not \models B \phi \supset B \psi$, ou seja, $\mathcal{M}, s \models B \phi$ e $\mathcal{M}, s \not \models B \psi$. Se $V$ é a função associada ao modelo $\mathcal{M}$, então $V(s)$ é uma 3 -interpretação para toda $s \in$ Sit. Mas toda 3-interpretação que satisfaz $\phi$ satisfaz também $\psi$, inclusive as $V\left(s^{\prime}\right)$ para $s^{\prime} \in \mathcal{B}$. Isso significa que, para todo $s^{\prime} \in \mathcal{B}, \mathcal{M}, s^{\prime} \models \phi$ implica em $\mathcal{M}, s^{\prime} \models \psi$, ou seja, que $\mathcal{M}, s \models B \phi$ implica em $\mathcal{M}, s \models B \psi$, contrário à hipótese.

$(\Leftarrow)$ Agora, suponha que $\models B \phi \supset B \psi$ mas $\phi \nvdash^{3} \psi$; isso significa que existe uma 3-interpretação $V^{\prime}$ que satisfaz $\phi$ mas não $\psi$. Considere o modelo dado por $\mathcal{M}=\langle$ Sit, $\mathcal{B}, V\rangle$, em que Sit $=\mathcal{B}=\{s\}$ e $V(s)=V^{\prime}$. Então $\mathcal{M}, s \models B \phi$ mas $\mathcal{M}, s \not \models B \psi$. Portanto, $\mathcal{M}, s \not \models B \phi \supset B \psi$, contrário à hipótese.

Nos trabalhos mais recentes de aproximações de lógicas, não mais se introduziram operadores específicos à crença, usando-se exclusivamente uma relação semelhante à $\models^{3}$ definida acima. As duas formas são essencialmente equivalentes. Uma propriedade extremamente importante dessa relação, mostrada acima, é o que pode ser chamado de correção: se $\phi \models^{3} \psi$, então $\phi \models \psi$. $^{4}$ Portanto, se for possível resolver o problema da inferência entre duas sentenças com a relação $\models^{3}$ (o que, via de regra, é mais fácil do que com a relação $\models$ ), então o problema da inferência dessas mesmas duas sentenças com a relação $\models$ também está solucionado.

${ }^{4} \mathrm{~A}$ relação desse conceito de correção com aquele apresentado na Seção 2.1.1 é a seguinte. Como a Lógica Proposicional Clássica é consistente, não acontece $\models \phi$ e $\models \neg \phi$ para nenhuma $\phi$. Como $\models^{3} \phi$ implica em $\models \phi$, também não pode acontecer $\models^{3} \phi \mathrm{e} \models^{3} \neg \phi$ para nenhuma $\phi$. 


\subsection{Compilação de Conhecimento de Selman e Kautz}

Os estudos da aplicação de lógica a modelos computacionais revelaram uma troca direta entre tratabilidade e expressividade. Ou seja, quanto mais tratável a linguagem, menor sua expressividade, e vice-versa. Bart Selman e Henry Kautz, em seu artigo "Knowledge compilation using Horn approximations" [SK91], dizem que, em geral, a fim de se obter um sistema de representação computacionalmente eficiente, ou se restringe o poder expressivo da linguagem para representação do conhecimento ou se usa um mecanismo de inferência incompleto. Na primeira abordagem, a linguagem de representação é freqüentemente muito limitada para aplicações práticas. A segunda abordagem envolve ou um raciocínio de recursos limitado ou a introdução de uma semântica não tradicional.

Como uma possível terceira solução para o problema, eles sugerem um método que envolve a aproximação das teorias de lógicas proposicionais por meio de teorias Horn. Como se sabe, testar se $\phi \models \psi$ na Lógica Proposicional Clássica, em que $\phi$ e $\psi$ são sentenças, é um problema co-NP-completo. Entretanto, quando $\phi$ é um conjunto de cláusulas de Horn (em uma cláusula de Horn, no máximo um literal é positivo, todos os outros sendo negações de átomos) e $\psi$ está na CNF, o problema é solúvel num tempo polinomial [SK91]. Eles chamaram seu método de compilação de conhecimento com aproximações Horn.

Seja $M(\phi)$ o conjunto de todos os modelos ${ }^{5}$ da sentença $\phi$ (que se assume estar em CNF, ou seja, ser um conjunto de cláusulas). Definem-se limites inferior e superior Horn, respectivamente $\phi_{l b}$ e $\phi_{u b}$, como subconjuntos de sentenças Horn que satisfaçam

$$
M\left(\phi_{l b}\right) \subseteq M(\phi) \subseteq M\left(\phi_{u b}\right) .
$$

$\mathrm{Na}$ ordem parcial representada pela relação $\subseteq$, podem existir supremos e ínfimos. Nesse caso em particular, sempre haverá um único ínfimo dos conjuntos $\phi_{u b}$ módulo equivalência lógica (denotado por $\phi_{l u b}$ ), mas possivelmente vários supremos dos conjuntos $\phi_{l b}$ (denotados por $\phi_{g l b}$ ). Isso segue do fato que a união de dois limites superiores Horn, $\phi_{u b}^{\prime}$ e $\phi_{u b}^{\prime \prime}$, é um limite superior Horn contido nos outros dois (ou seja, tem por modelos a intersecção do modelo de ambos, $\left.M\left(\phi_{u b}^{\prime} \cup \phi_{u b}^{\prime \prime}\right)=M\left(\phi_{u b}^{\prime}\right) \cap M\left(\phi_{u b}^{\prime \prime}\right)\right)$, mas a intersecção de dois limites inferiores Horn não é um limite inferior Horn que contém os outros dois [SK91].

No restante de seu artigo, Selman e Kautz demonstram uma série de teoremas e apresentam algoritmos que são úteis para a determinação de $\phi_{l u b}$ ou de um dos $\phi_{g l b}$. Mas, aqui, o que é importante é a idéia por trás desses conceitos. Suponha que, dado um conjunto (finito) $\phi$ de cláusulas, sejam determinados $\phi_{l u b}$ e $\phi_{g l b}$. Tome uma sentença $\psi$ em CNF. Se $\phi_{l u b} \models \psi$ (algo cuja determinação leva apenas tempo polinomial), podemos ter certeza que $\phi \models \psi$. Diz-se que $\phi_{l u b}$ mantém a correção da inferência. Dualmente, se $\phi_{g l b} \not \models \psi$, então também

${ }^{5}$ Uma definição do que é modelo de uma sentença é dada no Capítulo 2. 
$\phi \not \models \psi$. Diz-se que $\phi_{\text {glb }}$ mantém a completude da inferência. Não há como garantir que uma dessas duas situações ocorra, infelizmente. Será visto adiante que as idéias subjacentes ao método dos $\phi_{l u b}$ dão origem às aproximações de lógicas "por baixo", e as subjacentes ao método dos $\phi_{g l b}$ dão origem às aproximações de lógicas "por cima".

\subsection{O Conceito de Aproximações de Lógicas}

Embora o sistema $\mathbf{L}$ de Levesque tenha motivações filosóficas, enquanto a compilação de conhecimento de Selman e Kautz tem motivações computacionais, ambos visam um objetivo comum: resolver problemas que surgem como conseqüência da onisciência lógica, simplificando-os. Cadoli e Schaerf encontraram nessas abordagens a inspiração para um novo método, e na base desse método encontra-se o conceito de aproximaçôes de lógicas.

Nota-se nas abordagens de Levesque por um lado, e de Selman e Kautz por outro a falta do poder de incrementabilidade. Em outras palavras, utiliza-se o procedimento para tentar achar a solução do problema. Se essa solução não é encontrada, deve-se voltar à formulação original do problema para resolvê-lo; na melhor das hipóteses, os resultados negativos obtidos podem ser usados para diminuir o espaço de busca.

Um procedimento como o de Levesque enfraquece o poder de inferência de sentenças quando elas estão no escopo do operador $B$ (ou de ambos os lados da relação $\models^{3}$ ). Mesmo assim, o sistema $\mathbf{L}$ ainda pode ser considerado, legitimamente, uma lógica. Mas o que é uma lógica? E como é possível incrementar uma lógica para outra com maior poder de inferência?

"A melhor maneira de descobrir sobre o que a lógica trata é simplesmente fazendo lógica", escreveu A. N. Prior em [Pri62]. Não é o objetivo deste trabalho dar uma definição final de lógica, mas apenas uma que sirva a seus propósitos. No caso, há duas possíveis abordagens que são equivalentes ${ }^{6}$ mas que podem fornecer intuições distintas. Em uma, identificamos a lógica com o conjunto de seus teoremas. Por exemplo, na Lógica Proposicional Clássica, $C, \phi \supset \phi$ é um elemento. Outra possibilidade é identificar a lógica com a relação de inferência $\models$, definida no produto cartesiano da família dos subconjuntos finitos de sentenças pelo conjunto de sentenças. Como exemplo, $\{\phi, \phi \supset \psi\} \models \psi$ é um elemento de $C$.

As lógicas que aproximam uma determinada lógica $\mathcal{L}$ podem ser visualizadas, seguindo a apresentação de Finger e Wassermann em [FW02, FW04], como uma seqüência $\left\{\mathcal{L}_{i}\right\}$ tal que

$$
\left\|\mathcal{L}-\mathcal{L}_{0}\right\| \supseteq\left\|\mathcal{L}-\mathcal{L}_{1}\right\| \supseteq \cdots \supseteq \emptyset,
$$

em que $\left\|\mathcal{L}-\mathcal{L}_{i}\right\|=\left(\mathcal{L}-\mathcal{L}_{i}\right) \cup\left(\mathcal{L}_{i}-\mathcal{L}\right)$ é a diferença simétrica entre os dois conjuntos $\mathcal{L}$ e $\mathcal{L}_{i}$. De especial interesse são aquelas lógicas $\mathcal{L}_{i}$ que estão contidas

\footnotetext{
${ }^{6} \mathrm{~A}$ equivalência ocorre necessariamente apenas no caso da Lógica Clássica. Em lógicas não-clássicas essas noções podem divergir.
} 
na lógica $\mathcal{L}$ original. A seqüência acima, então, implica que

$$
\mathcal{L}_{0} \subseteq \mathcal{L}_{1} \subseteq \cdots \subseteq \mathcal{L}
$$

É possível também, dualmente, considerar lógicas $\mathcal{L}_{i}$ que sempre contêm a lógica $\mathcal{L}$, dando origem à seqüência

$$
\mathcal{L} \subseteq \cdots \subseteq \mathcal{L}_{1} \subseteq \mathcal{L}_{0}
$$

Aproximações de lógicas que usam o primeiro tipo de seqüência acima são chamadas aproximações por baixo, e o segundo tipo aproximações por cima. Como será visto no Capítulo 3, apenas as aproximações por baixo enfraquecem a onisciência lógica no método de Cadoli-Schaerf (e, a fortiori, nos métodos subseqüentes). Por essa razão, aqui só serão analisadas com detalhe as aproximações por baixo.

O problema da incrementabilidade é resolvido como segue. Dado $\Gamma, \phi$, deseja-se verificar se $\Gamma \models \phi$. Começa-se com uma lógica $\mathcal{L}_{0}$ bem simples, com poder de inferência extremamente limitado e, portanto, bastante tratável. Se $\Gamma \models_{\mathcal{L}_{0}} \phi$, então $\Gamma \models \phi$ (esse é o Teorema da Correção). Caso contrário, constrói-se outra lógica, $\mathcal{L}_{1} \supseteq \mathcal{L}_{0}$, que contém a anterior mas que é mais poderosa. Se ela não resolve o problema, constrói-se uma terceira lógica $\mathcal{L}_{2} \supseteq \mathcal{L}_{1}$, e assim por diante. Para aplicações práticas, é necessário apenas um número finito de passos para resolver os problemas. Então, eventualmente chega-se ao resultado (e isto distingue as aproximações de lógicas de abordagens como a de Levesque ou de Selman e Kautz).

\subsection{Objetivos}

O principal objetivo deste trabalho é realizar a extensão do procedimento de aproximações de lógicas de Marcelo Finger e Renata Wassermann (apresentado no Capítulo 5) para lógicas modais. Um segundo objetivo é formalizar o procedimento de Finger-Wassermann em uma lógica modal do conhecimento, como foi feito por Cadoli e Schaerf em seu procedimento (ver Seção 3.2).

\subsection{Estrutura da dissertação}

Esta dissertação de mestrado possui a seguinte estrutura:

Capítulo 1: Discute-se o panorama teórico que serviu de pano de fundo para o desenvolvimento do conceito de aproximações de lógicas (Seção 1.1 e Seção 1.2); define-se de maneira geral esse conceito (Seção 1.3).

Capítulo 2: São apresentados os resultados preliminares essenciais à compreensão do resto do texto: Lógica Proposicional Clássica e Tableaux de Smullyan (Seção 2.1) e Lógicas Proposicionais Modais e SST ("Single Step Tableaux") (Seção 2.2). 
Capítulo 3: O trabalho de Cadoli-Schaerf, pioneiro na área de aproximações de lógicas, é estudado na medida necessária a este texto. Sua semântica com alguns resultados são dados na Seção 3.1; a formalização desses resultados em uma lógica modal do conhecimento é dada na Seção 3.2; a extensão de seu procedimento para lógicas modais é dada na Seção 3.3.

Capítulo 4: Fabio Massacci trabalhou uma extensão do procedimento de Cadoli-Schaerf, tanto para Lógica Clássica com linguagem completa (todos os operadores clássicos e nenhuma restrição ao formato das sentenças), como para Lógica Modal. Os resultados em Lógica Clássica, bem como a teoria da prova a partir dos Tableaux de Smullyan, são descritos na Seção 4.1. Os resultados em Lógica Modal, com a correspondente teoria da prova no contexto dos SST, são descritos na Seção 4.2.

Capítulo 5: O trabalho de Marcelo Finger e Renata Wassermann realiza uma outra extensão do procedimento de Cadoli-Schaerf para Lógica Clássica. A semântica por eles sugerida é dada na Seção 5.1, e a correspondente teoria da prova no contexto dos KE-tableaux é dada na Seção 5.2.

Capítulo 6: São apresentadas extensões do procedimento de Finger-Wassermann para Lógica Modal. Na Seção 6.1, é dada uma semântica que regula o comportamento do operador $\neg$ e o limite máximo de introspecção (que controla o número de interações modais). A Seção 6.2 mostra uma correspondente teoria da prova no contexto dos SST. A Seção 6.3 apresenta uma semântica mais fraca e correspondente teoria da prova, e a Seção 6.4 sugere uma semântica mais geral e possíveis estudos posteriores.

Capítulo 7: Formaliza-se a semântica de Finger-Wassermann em uma lógica modal do conhecimento, como feito por Cadoli e Schaerf na Seção 3.2, demonstrando-se alguns resultados associados.

Capítulo 8: Na Seção 8.1, analisam-se os resultados obtidos, bem como os apresentados na dissertação como um todo. Na Seção 8.2 são sugeridas possíveis direções para trabalhos futuros. 


\section{Capítulo 2}

\section{Preliminares}

O objetivo deste capítulo é, principalmente, fixar a notação utilizada, assim como enunciar resultados e definições padrão.

\subsection{Lógica Proposicional Clássica}

\subsubsection{Notação, Sintaxe e Semântica}

O estudo de um sistema formal se subdivide em duas partes: estudos de sintaxe (forma) e semântica (significado).

Para [Sch67], a determinação da sintaxe de um sistema formal é feita em três estágios. Primeiramente, especificam-se quais são os sinais a serem utilizados. No caso da Lógica Proposicional Clássica (resumidamente, $L P C$ ), utilizam-se os seguintes sinais:

- os símbolos proposicionais

$$
p_{0}, p_{1}, p_{2}, p_{3}, \ldots
$$

ou seja, uma seqüência enumerável de símbolos distintos, que podem ser chamados de átomos. As letras $q$ e $r$, com ou sem subscritos, também servem para designar elementos dessa seqüência.

- os operadores lógicos

$$
\neg, \wedge, \vee, \supset, \equiv
$$

$\neg$ (negação) é um operador lógico unário, ao passo que os outros (respectivamente, conjunção, disjunção, implicação e equivalência) são operadores binários

- parêntesis (, ); estes servem apenas para fins de pontuação 
Algumas seqüências finitas dos sinais apresentados acima são escolhidas (intuitivamente, as seqüências escolhidas são as que remetem a algum sentido). Chamam-se a essas sequiências de sentenças. Usualmente, as sentenças são definidas por recursão, como segue ${ }^{1}$ :

\section{Definição Recursiva de Sentenças}

1. $p_{i}$ é uma sentença, para todo $i \in \mathbb{N}$

2. $\neg \phi$ (lê-se "não $\phi$ ") é uma sentença se $\phi$ é uma sentença

3. $\phi \wedge \psi$ (lê-se " $\phi$ e $\psi$ ") é uma sentença se $\phi$ e $\psi$ são sentenças

4. $\phi \vee \psi$ (lê-se " $\phi$ ou $\psi$ ") é uma sentença se $\phi$ e $\psi$ são sentenças

5. $\phi \supset \psi$ (lê-se " $\phi$ implica em $\psi$ ") é uma sentença se $\phi$ e $\psi$ são sentenças

6. $\phi \equiv \psi$ (lê-se " $\phi$ equivale a $\psi$ ") é uma sentença se $\phi$ e $\psi$ são sentenças

O segundo estágio na determinação de um sistema formal é a escolha de um certo conjunto de sentenças em particular, que são chamadas de axiomas. Em geral, as sentenças escolhidas são o mais simples possível e remetem a um sentido de uma verdade evidente por si só. Mas não é uma exigência para um sistema formal. De fato, sempre que se tentou dar uma definição precisa de "verdade evidente por si só", caiu-se em problemas de subjetividade e circularidade de definições. Portanto, não existe mais o requerimento de simplicidade e evidência por si só para axiomas, a fim de se trabalhar da maneira mais objetiva possível.

Para $L P C$, deve valer o seguinte grupo de axiomas, adaptados de [Euka63]:

Axiomas da lógica Proposicional Clé́ssica

1. $(p \supset q) \supset((q \supset r) \supset(p \supset r))$

2. $(\neg p \supset p) \supset p$

3. $\neg p \supset(p \supset q)$

Além disso, valem as seguintes definições (discussões sobre essas definições em particular e do significado das definições em lógica podem ser encontradas em [Łuka63]):

\section{Definições da LóGica Proposicional Clássica}

$D f_{\vee} . \phi \vee \psi$ é o mesmo que $\neg \phi \supset \psi$

$\mathrm{Df}_{\wedge} . \phi \wedge \psi$ é o mesmo que $\neg(\phi \supset \neg \psi)$

\footnotetext{
${ }^{1}$ Existe um problema técnico nas sentenças a seguir: é a forma de usar os parênteses de maneira a garantir uma única leitura das seqüências; neste texto assume-se a forma padrão de associação implícita de parênteses e a associação segundo o contexto em que as sentenças são empregadas; a esse respeito, remete-se a [Kle52] e a [Smu68].
} 
$\mathrm{Df}_{\equiv .} \phi \equiv \psi$ é o mesmo que $(\phi \supset \psi) \wedge(\psi \supset \phi)$

No sistema de $L P C$ apresentado aqui, definem-se todos os operadores lógicos em termos de $\neg$ e $\supset$, que são chamados de operadores básicos. Sistemas distintos podem considerar como básicos outros operadores.

Todos os axiomas de um sistema formal são teoremas, mas nem todos os teoremas são axiomas. Intuitivamente, um teorema é uma asserção que pode ser demonstrada, ou seja, cuja verdade é necessária se os axiomas são assumidos. Como o objetivo do estudo de sistemas formais é justamente a análise dos tipos de argumentos que se aceitam como demonstrações, é necessária a redução do conceito de teorema a um sentido puramente sintático. Assim, teoremas são certos tipos de sentenças. Isto ficará mais claro adiante.

Por fim, o último estágio é a escolha de regras de inferência. Conforme [Sch67], uma regra de inferência diz sobre que condições uma sentença, chamada de conclusão, pode ser inferida como teorema de outras sentenças que são teoremas, chamadas de hipóteses.

Para $L P C$, devem valer as seguintes regras de inferência:

Modus Ponens: se $\phi$ e $\phi \supset \psi$ são teoremas, então $\psi$ é um teorema

Substituição Uniforme: se $\phi$ é um teorema, então qualquer sentença obtida de $\phi$ substituindo certos átomos por sentenças de maneira uniforme (isto é, átomos iguais são substituídos por sentenças iguais) é um teorema

Com os axiomas e as regras de inferência, [Sch67] define o que é um teorema como segue: um teorema é ou um axioma, ou uma sentença obtida de outros teoremas a partir das regras de inferência.

O esquema de axiomas e as regras de inferência escolhidos até agora não são tão importantes, desde que eles dêem ao sistema formal as características de correção e completude, que serão explicadas após a definição da semântica.

A semântica de um sistema axiomático formal é determinada especificando a que estruturas matemáticas se relaciona a sintaxe. Essas estruturas são chamadas de modelos do sistema formal.

Definição 2.1 Um modelo $V$ de LPC é uma função $V: A \rightarrow\{0,1\}$ do conjunto $A$ de átomos ao conjunto $\{0,1\}$ que é estendida para o conjunto $\mathcal{L}_{C}$ de todas as sentenças como segue:

1. $V(\neg \phi)=1$ sse $V(\phi)=0$

2. $V(\phi \wedge \psi)=1$ sse $V(\phi)=V(\psi)=1$

3. $V(\phi \vee \psi)=0$ sse $V(\phi)=V(\psi)=0$

4. $V(\phi \supset \psi)=0$ sse $V(\phi)=1$ e $V(\psi)=0$

5. $V(\phi \equiv \psi)=1$ sse $V(\phi)=V(\psi)$ 
A função $V$ é chamada de valoração. Intuitivamente, $V$ é uma avaliação de verdade das sentenças do sistema formal: 1 é o valor "verdadeiro" e 0 é o valor "falso". A valoração $V$ é um modelo para uma sentença $\phi$ ( $V$ satisfaz $\phi, V$ é um modelo de $\phi$ ) se $V(\phi)=1$. Se $\Gamma$ é um conjunto de sentenças, $V$ é um modelo de $\Gamma$ se $V$ for um modelo para cada sentença em $\Gamma$. Uma sentença válida é uma sentença $\phi$ (utiliza-se a notação $\models \phi$ ) cujas funções verdade sempre atribuem o valor 1, para qualquer valoração das sentenças que a compõem. Dessas sentenças também se diz que são tautologias. Assim, toda valoração é um modelo para as tautologias. Dado um conjunto $\Gamma=\left\{\phi_{1}, \phi_{2}, \ldots, \phi_{n}\right\}$ de sentenças e uma sentença $\psi$, diz-se que $\Gamma$ implica logicamente ou tautologicamente $\psi$ (e denotase $\Gamma \models \psi$ ) quando toda valoração que satisfizer todas as sentenças em $\Gamma$ satisfizer também $\psi$. Um teorema de $L P C$, chamado teorema da dedução, afirma que se $\Gamma \models \psi$, então $\models \phi_{1} \wedge \phi_{2} \wedge \cdots \wedge \phi_{n} \supset \psi$, ligando a noção de implicação lógica à noção de sentenças válidas.

Quando foram discutidos os axiomas de $L P C$ e as regras de inferência, anunciou-se que esses não eram importantes desde que o sistema axiomático formal possuísse as características de correção (ou consistência) e completude. Um sistema é correto quando, a partir dele, não se pode provar dois teoremas, um dos quais tem a forma $\phi$ e o outro a forma $\neg \phi$. Um sistema é completo quando nele, dada uma sentença $\phi$ qualquer, se $\phi$ não for um teorema, sua adição ao sistema implica em uma contradição (ou seja, torna-se possível demonstrar no novo sistema um par de sentenças $\psi$ e $\neg \psi)$. Pode-se provar que $L P C$ é correta e completa (veja, por exemplo, [Łuka63]; para uma apresentação alternativa de axiomas com demonstração de correção e completude, veja [HA50]). Isso vem a ser equivalente a dizer que todo teorema de $L P C$ é uma tautologia, e toda tautologia é um teorema de $L P C$. Como existe um procedimento mecânico e finito para determinar se uma determinada sentença é ou não tautologia, algumas formalizações de lógica assumem como axiomas todas as tautologias. Todas as formalizações de $L P C$ que tenham por teoremas exatamente as tautologias são equivalentes.

\subsubsection{Tableaux de Prova}

Tableaux semânticos representam um outro paradigma de teoria da prova, em contraposição aos sistemas de Hilbert mais usuais (apresentados na seção anterior), e vêm gozando de grande popularidade nas últimas décadas, principalmente após a publicação de [Smu68], do qual é retirada a maior parte da informação contida nesta seção.

Dada uma sentença $\phi$ qualquer, pode-se assinalar $\phi$, ou seja, antepor a $\phi$ os sinais $T$ ou $F$, formando uma sentença assinalada $T \phi$ ou $F \phi$. Nesse contexto, costuma-se utilizar a expressão "sentença não assinalada" para $\phi$. Intuitivamente, $T \phi$ significa "a sentença $\phi$ é verdadeira" e $F \phi$ significa "a sentença $\phi$ é falsa". Assim, $T \phi$ é verdadeira sse $\phi$ for verdadeira, e $F \phi$ é verdadeira sse $\phi$ for falsa.

A conjugada de uma sentença assinalada $T \phi$ é $F \phi$ e a conjugada de uma sentença assinalada $F \phi$ é $T \phi$. Em geral, se $\varphi$ é uma sentença assinalada, $\bar{\varphi}$ é 
sua conjugada.

Antes de definir tableaux, são necessárias algumas observações. Uma árvore binária é um conjunto de nós, um dos quais é a origem e que, para cada nó, associa como sucessores no máximo dois outros nós. Todo nó com exceção da origem é sucessor de um, e apenas um, outro nó. A origem é ligada a qualquer nó $n$ da árvore por uma seqüência finita de nós em que o primeiro termo é a origem, cada termo seguinte é sucessor do anterior e o último termo é $n$. Um nó sem sucessores é chamado de terminal. Uma seqüência maximal de nós que começa na origem e que tem, para cada elemento posterior da seqüência, um dos sucessores do elemento anterior é chamado de ramo da árvore. Nos tableaux, cada nó conterá uma sentença assinalada.

Existem diversos tipos de tableaux, e a definição que será dada a seguir serve para muitos deles. Em essência, o que distingue um tableau de outro é o conjunto de regras que permite adicionar sentenças novas aos ramos do tableau.

Dada um conjunto de sentenças assinaladas $\Gamma$, um tableau para $\Gamma$ é uma árvore binária definida recursivamente como segue:

- um nó contendo apenas uma sentença assinalada $\varphi \in \Gamma$ é um tableau; esse nó é a origem

- se $R$ é um ramo com nó terminal de um tableau $\mathcal{T}$, a árvore obtida de $\mathcal{T}$ adicionando a sentença assinalada $\varphi \in \Gamma$ como sucessor do nó terminal de $R$ (caso ela já não esteja em $R$ ) é um tableau

- se $R$ é um ramo com nó terminal de um tableau $\mathcal{T}$, a árvore obtida de $\mathcal{T}$ adicionando como sucessor do nó terminal de $R$ uma sentença obtida a partir da aplicação das regras do tableau (caso ela já não esteja em $R$ ) é um tableau

O ramo de um tableau fecha quando nele aparecem uma sentença assinalada e sua conjugada, ou seja, uma contradição; o tableau fecha se todos seus ramos fecham.

A demonstração de uma sentença não assinalada $\phi$ qualquer é feita da seguinte forma. Assinala-se $\phi$ como falsa, isto é, como $F \phi$. Constrói-se o tableau para $\{F \phi\}$. Se o tableau fechar, a sentença foi provada; caso contrário, a prova falhou. Para provar que um conjunto finito $\Gamma=\left\{\phi_{1}, \phi_{2}, \ldots, \phi_{n}\right\}$ de sentenças não assinaladas implica tautologicamente uma outra sentença (também não assinalada) $\psi$ (ou, utilizando uma notação desenvolvida por Gentzen [Gen69], para provar o seqüente $\Gamma \vdash \psi$ ), assinalam-se as sentenças de $\Gamma$ como verdadeiras e a sentença $\psi$ como falsa, e tenta-se fechar o tableau para o conjunto $\left\{T \phi_{1}, T \phi_{2}, \ldots, T \phi_{n}, F \psi\right\}$.

Assumindo que uma sentença assinalada $\varphi$ é verdadeira, obtêm-se as chamadas conclusões diretas da mesma. Por exemplo, a sentença $T \phi \wedge \psi$ só pode ser verdadeira se $\phi$ e $\psi$ forem. Assim, aceitar $T \phi \wedge \psi$ implica na aceitação de $T \phi$ e de $T \psi$. Por outro lado, se a sentença $T \phi \vee \psi$ é verdadeira, então ou $T \phi$ ou $T \psi$ é verdadeira (não necessariamente as duas).

Smullyan distinguiu dois tipos de sentenças assinaladas, segundo o tipo de conclusões diretas que delas se tiram. Um dos tipos, em que se encaixa $T \phi \wedge \psi$, 
foi designado por sentenças do tipo $\alpha$, e as conclusões diretas de $\alpha_{1}$ e $\alpha_{2}$. A aceitação de sentenças $\alpha$ implica na aceitação simultânea de $\alpha_{1}$ e $\alpha_{2}$. O segundo tipo, em que se encaixa $T \phi \vee \psi$, foi designado por sentenças do tipo $\beta$, e as conclusões diretas de $\beta_{1}$ e $\beta_{2}$. A aceitação de sentenças $\beta$ implica na aceitação ou de $\beta_{1}$ ou de $\beta_{2}$ (não necessariamente na aceitação das duas). Sentenças do tipo $T \neg \phi$ e $F \neg \phi$ podem pertencer a qualquer um dos dois tipos, e aqui é preferida a abordagem de Fabio Massacci, que em [Mas98a] as considera como pertencentes a uma terceira classe, designada por neg, cuja (única) conseqüência é designada por pos. Na Tabela 2.1 sumarizam-se os tipos de sentenças.

\begin{tabular}{|ccc|}
\hline$\alpha$ & $\alpha_{1}$ & $\alpha_{2}$ \\
\hline$T \phi \wedge \psi$ & $T \phi$ & $T \psi$ \\
$F \phi \vee \psi$ & $F \phi$ & $F \psi$ \\
$F \phi \supset \psi$ & $T \phi$ & $F \psi$ \\
\hline
\end{tabular}

\begin{tabular}{|cc|}
\hline$n e g$ & $p o s$ \\
\hline$T \neg \phi$ & $F \phi$ \\
$F \neg \phi$ & $T \phi$ \\
\hline
\end{tabular}

\begin{tabular}{|ccc|}
\hline$\beta$ & $\beta_{1}$ & $\beta_{2}$ \\
\hline$F \phi \wedge \psi$ & $F \phi$ & $F \psi$ \\
$T \phi \vee \psi$ & $T \phi$ & $T \psi$ \\
$T \phi \supset \psi$ & $F \phi$ & $T \psi$ \\
\hline
\end{tabular}

Tabela 2.1: Notação unificadora de Smullyan

Os protótipos dos tableaux utilizados neste texto serão os chamados Tableaux de Smullyan, apresentados em [Smu68]. Esses tableaux têm as seguintes regras, chamadas regras de expansão:

T1. para toda sentença assinalada da forma $\alpha$, adicionar $\alpha_{1}$ e $\alpha_{2}$ ao ramo do tableau

T2. para toda sentença da forma neg, adicionar pos ao ramo do tableau

T3. para toda sentença assinalada da forma $\beta$, ramificar em dois o tableau, adicionando $\beta_{1}$ a um ramo e $\beta_{2}$ ao outro ramo do tableau

As regras enunciadas acima são sumarizadas na Tabela 2.2 .

$$
\begin{array}{lll}
\frac{\alpha}{\alpha_{1}} & \frac{n e g}{p o s} & \frac{\beta}{\beta_{1} \beta_{2}} \\
\alpha_{2} & & \\
\hline
\end{array}
$$

Tabela 2.2: Regras de Smullyan para Lógica Clássica

Uma sentença assinalada $\varphi$ é reduzida em um ramo $R$ (segundo o Tableau de Smullyan) se

R1. $\varphi$ é um átomo assinalado

R2. $\varphi$ é uma sentença $\alpha$ e tanto $\alpha_{1}$ como $\alpha_{2}$ estão em $R$

R3. $\varphi$ é uma sentença neg e pos está em $R$

R4. $\varphi$ é uma sentença $\beta$ e ou $\beta_{1}$ ou $\beta_{2}$ está em $R$ 
Quando todas as sentenças em um ramo estão reduzidas, não há mais o que se fazer nele; se não se chegou a uma contradição, a prova falhou, e o tableau pode ser utilizado para construir um contra-modelo da sentença (ou seja, um modelo para sua negação).

Na Figura 2.1 é apresentado um exemplo de aplicação dos Tableaux de Smullyan, extraído de [Smu68]. No exemplo, demonstra-se a tautologia $p \vee$ $(q \wedge r) \supset(p \vee q) \wedge(p \vee r)$. As cruzes no final de cada ramo indicam que ele fechou. As sentenças (2)-(3) são obtidas de (1) pela regra $\alpha$. As sentenças (4)-(5), de (3) por $\beta$. As sentenças (6)-(7), de (5) por $\alpha$. As sentenças (8)-(9) e (10)-(11), de (4) e (7), respectivamente, por $\beta$. Por fim, as sentenças (12)-(13), (14)-(15), (16)-(17) e (18)-(19) são obtidas respectivamente de (8), (9), (10) e (11) por aplicação da regra $\alpha$.

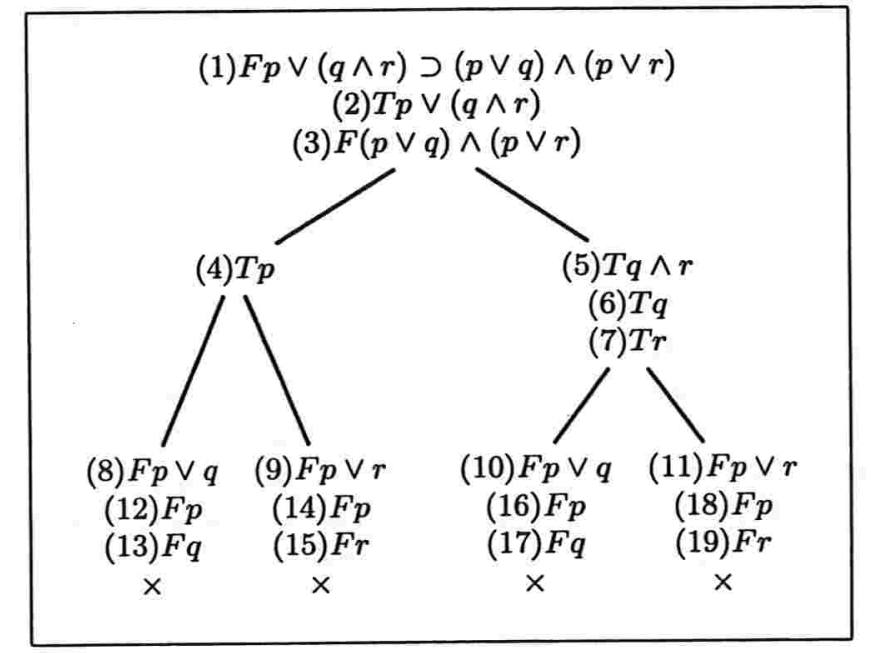

Figura 2.1: Um exemplo de aplicação dos Tableaux de Smullyan

Tableaux semânticos fornecem, como se disse, uma teoria para provar sentenças. Há que se verificar se essa teoria de prova é correta e completa com relação à semântica intencionada. Diz-se que um determinado tableau é correto com relação a determinada semântica se toda inferência realizada por meio do tableau pode ser feita também pela semântica. Uma vez que é impossível, pela semântica, a inferência de duas sentenças, uma das quais tem a forma $\phi$ e a outra $\neg \phi$, essa noção de correção liga-se àquela dada na seção anterior. Um tableau é completo com relação a determinada semântica se toda inferência realizada pela semântica pode ser feita também por meio do tableau. Como só se inferem tautologias ou implicações tautológicas na semântica, isso implica que o tableau prova todas as tautologias, o que liga essa noção de completude àquela dada na seção anterior.

A demonstração da correção dos diferentes tableaux é semelhante. No caso dos Tableaux de Smullyan, ela é feita como segue. Supõe-se que exista uma valoração $V$ que satisfaça a origem do tableau (ou a negação do seqüente que 
se deseja demonstrar). Prova-se que a aplicação das regras T1-3 dá origem a um novo nó em que $V$ continua sendo satisfeita. Por exemplo, se $V$ é válida num ramo $R$ e é aplicada a regra T1, as duas sentenças $\alpha_{1}$ e $\alpha_{2}$ são satisfeitas por $V$; se $V$ é válida num ramo $R$ e é aplicada a regra T3, pelo menos uma das duas sentenças $\beta_{1}$ e $\beta_{2}$ é satisfeita por $V$ (no caso de um tableau arbitrário, deve-se mostrar que a aplicação das regras do tableau permitem o aparecimento de ao menos um nó satisfatível por $V$ ). Assim, se o tableau fecha, ter-se-ia a existência de uma valoração $V$ que satisfaz um par de sentenças contraditórias (pois em todos os ramos aparecem sentenças dessa forma). Isso é impossível, e portanto não há valoração $V$ que possa satisfazer a origem (ou a negação do seqüente).

Proposição 2.2 (Correção) Seja $\Gamma$ um conjunto finito de sentenças. Suponha que um tableau para o seqüente $\Gamma \vdash \psi$ feche. Então $\Gamma \models \psi$.

A demonstração da completude de um tableau é feita da seguinte maneira. Supõe-se, por contradição, que um tableau de determinada tautologia não feche, mesmo estando todos seus ramos reduzidos. Isso significa que algum ramo do tableau permaneceu aberto. Esse ramo é utilizado para a construção de um contra-modelo para a negação da tautologia. Ramos cujas sentenças estão reduzidas são conjuntos de Hintikka, e o chamado lema de Hintikka assegura que tais conjuntos são satisfatíveis por alguma valoração $V$. Essa valoração associa a cada átomo $p$ tal que $T p$ esteja no conjunto o valor 1 , e a cada $p$ tal que $F p$ esteja no conjunto o valor 0 ; o fato de o conjunto ser de Hintikka (ou seja, do ramo ser aberto) assegura que não surgem contradições sob tal valoração. O fato de a negação da tautologia ser satisfatível contradiz o fato de que a tautologia é tautologia, o que é absurdo. Considerações parecidas valem as demonstrações de seqüentes.

Proposição 2.3 (Completude) Seja $\Gamma$ um conjunto finito de sentenças. Suponha que $\Gamma \models \psi$. Entẫo, qualquer tableau para o seqüente $\Gamma \vdash \psi$ fecha.

Correção e completude são demonstrados com detalhes em [Smu68].

A existência de diversos tableaux é principalmente devida ao seguinte problema, observado por Marcello D'Agostino em [D'A92, D'A99]. Parte do interesse sobre os tableaux parte do fato que, aparentemente, eles fornecem um método mais eficiente para demonstrar tautologias. $\mathrm{O}$ método tradicional das tabelasverdade para decisão de $L P C$ exige que, para testar uma tautologia com $n$ átomos, façam-se $2^{n}$ cálculos. Assim, com 10 átomos são feitos cerca de 1000 cálculos. Entretanto, D'Agostino demonstra que existem exemplos de tautologias com 10 átomos cujo Tableau de Smullyan contém no mínimo 3000000 de ramos! Uma das razões desse comportamento paradoxal está no fato que os Tableaux de Smullyan permitem muitas ramificações (uma para cada aplicação de uma regra $\beta$ ). Refinamentos posteriores desses tableaux procuram diminuir a quantidade de ramificações que surgem.

Não é objetivo deste trabalho a análise dos diversos tableaux e suas relativas complexidades computacionais, cuja literatura é extensa. Mas os trabalhos de 
Massacci e Finger-Wassermann, que serão discutidos adiante, utilizam tableaux com diferentes regras de expansão, e no resto desta seção será feita uma rápida análise desses tableaux. Entretanto, para o resto do texto, será assumido como protótipo de tableaux proposicionais os Tableaux de Smullyan.

Finger e Wassermann partem de uma modificação dos Tableaux de Smullyan introduzida por Marco Mondadori em [Mon88a, Mon88b] e extensivamente estudada por Marcello D'Agostino em [D'A90, D'A92], chamada KE-tableaux, com propriedades computacionais mais atraentes. Na Tabela 2.3 (extraída de [FW01]) sumarizam-se as regras KE para Lógica Clássica. Uma inspeção das regras mostra que apenas uma permite a ramificação do tableau: a regra $(P B)$.

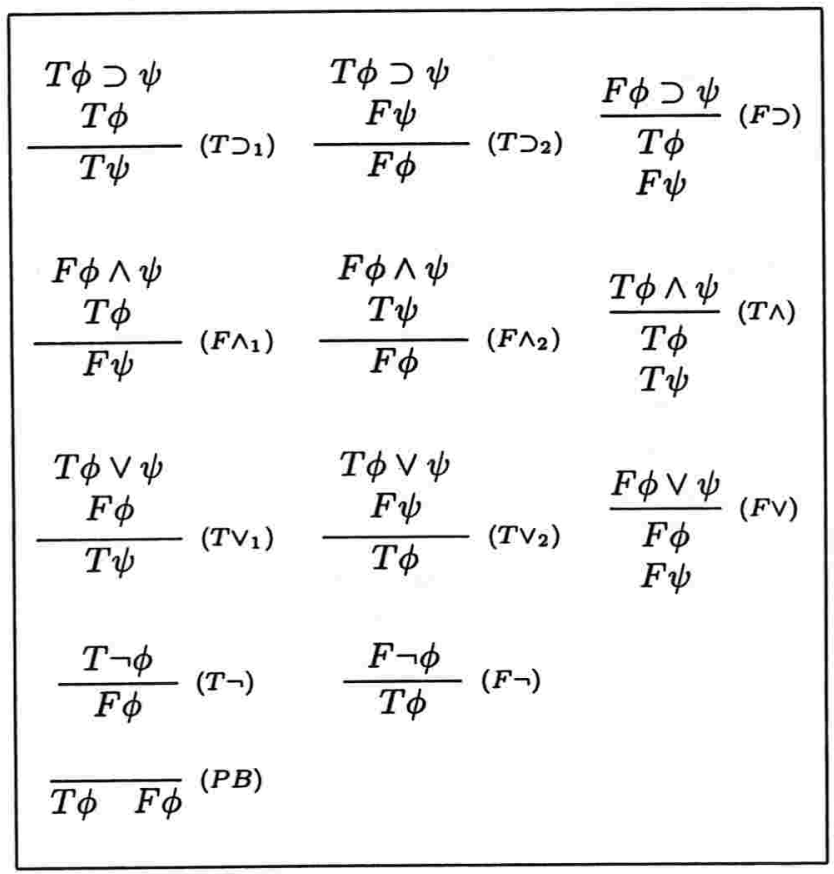

Tabela 2.3: Regras KE para Lógica Clássica

A regra da simplificação, utilizada por Massacci em seus tableaux, consiste no seguinte. Dadas duas sentenças assinaladas $\varphi$, $\varpi$, é possível reescrever $\varphi$ a partir de certas propriedades de $\varpi$, obtendo uma nova sentença que será denotada por $\varphi[\varpi]$ (ver explicação adiante). Se $\varphi$ e $\varpi$ estão presentes em um ramo, então adiciona-se $\varphi[\varpi]$ ao ramo e considera-se que $\varphi$ está reduzida no mesmo (a não ser, evidentemente, que $\varphi[\varpi]=\varphi$, pois nesse caso não há necessidade de adicionar $\varphi[\varpi]$ ao ramo). Para a definição precisa de $\varphi[\varpi]$, refere-se a [Mas98a]. Aqui, serão citados apenas alguns exemplos.

1. Suponha que $\varphi=T p \supset q$ e $\varpi=T p$. Então $\varphi[\varpi]$ é obtida considerando-se $p$ verdadeiro na sentença $p \supset q$ e reduzindo-a utilizando as regras boolea- 
nas (por exemplo, se $p$ é verdadeiro, então $p \supset q=q$; se $q$ é falso, então $p \vee q=p$; e assim por diante). Assim, $\varphi[\varpi]=T q$.

2. Suponha que $\varphi=F p \wedge(q \vee r)$ e $\varpi=T q \vee r$. Então $\varphi[\varpi]=T p$.

3. Suponha que $\varphi=T p \vee q$ e $\varpi=F r$. Então $\varphi[\varpi]=\varphi=T p \vee q$.

4. Suponha que $\varphi=T p \vee q$ e $\varpi=T q$. Então $p \vee q$ é verdadeira, e $\varphi[\varpi]$ é qualquer sentença válida, como por exemplo $\varphi=T p \vee \neg p$. Nesse caso, deve-se utilizar na sentença válida apenas átomos que estão dentro de $\varphi$.

Observa-se que tableaux com as regras $\alpha, n e g$, de simplificação e $(P B)$ generalizam os KE-tableaux, uma vez que cada uma das regras $\left(T \supset_{1}\right),\left(T \supset_{2}\right),\left(F \wedge_{1}\right)$, $\left(F \wedge_{2}\right),\left(T \vee_{1}\right)$ e $\left(T \vee_{2}\right)$ podem ser obtidas a partir da regra de simplificação. Massacci, entretanto, não utiliza $(P B)$.

\subsection{Lógicas Proposicionais Modais}

\subsubsection{Notação, Sintaxe e Semântica}

Os operadores lógicos que aparecem em $L P C$ possuem uma importante característica. Dada uma valoração $V$ sobre os átomos, a valoração sobre qualquer sentença obtida utilizando-se apenas os operadores clássicos está determinada $\left(S_{C} 1-5\right)$. Como observa [HC96], na lógica modal aparecem sentenças cuja verdade não depende somente da verdade de suas partes componentes, em particular dos átomos. Além dos operadores lógicos clássicos, há outros dois operadores unários, $\square$ (necessitação) e $\diamond$ (possibilitação). ${ }^{2}$ As sentenças são definidas por recursão como na Seção 2.1.1, com as duas regras adicionais

7. $\square \phi$ (lê-se "necessariamente $\phi$ ") é uma sentença se $\phi$ é uma sentença

8. $\diamond \phi$ (lê-se "possivelmente $\phi$ ") é uma sentença se $\phi$ é uma sentença

Sentenças com esses operadores são chamadas de modais, provavelmente porque ser necessariamente ou possivelmente verdade são pensados como modos ou maneiras pelas quais uma proposição é verdadeira [HC96].

Em [Che80], chamam-se lógicas modais normais os sistemas formais que possuem, além de todos os axiomas, definições e regras de inferência de $L P C$, pelo menos o seguinte axioma adicional:

$$
\text { K. } \square(p \supset q) \supset(\square p \supset \square q)
$$

e também a seguinte regra de inferência:

Regra da Necessitação: se $\phi$ é um teorema, então $\square \phi$ também é um teorema Além disso, vale a definição abaixo:

${ }^{2} \mathrm{O}$ emprego dos neologismos "necessitação" e "possibilitação" é inspirado pelo texto de Chellas [Che80], e é adequado quando as expressões linguísticas "necessariamente" e "possivelmente" são utilizadas como operadores. 
$\mathrm{Df}_{\diamond} . \diamond \phi$ é o mesmo que $\neg \square \neg \phi$

$\mathrm{Df}_{\diamond}$ define o operador $\diamond$ em termos do operador $\square$. O axioma $K$ caracteriza o operador $\square$ como normal.

Existem alguns princípios, ou axiomas, de lógicas modais normais que serão colocados na Tabela 2.4 para referência futura e que deixarão claros alguns aspectos da semântica que será exibida. O que distingue uma lógica modal de outras são os princípios que nela valem. Por exemplo, uma lógica modal chamada $\mathbf{T}$ tem por axiomas $K$ e $T$; outra, chamada $\mathbf{D}$, tem por axiomas $K$ e $D$. Toda lógica modal normal deve possuir o axioma $K$, mas os diferentes axiomas que são adicionados dão origem a diferentes lógicas, todas contidas na lógica modal normal mais geral, que possui apenas $K$ por axioma. ${ }^{3}$

\begin{tabular}{|c|c|}
\hline Axioma & Sentença \\
\hline$K$ & $\square(p \supset q) \supset(\square p \supset \square q)$ \\
$T$ & $\square p \supset p$ \\
$D$ & $\square p \supset \diamond p$ \\
4 & $\square p \supset \square \square p$ \\
5 & $\diamond p \supset \square \diamond p$ \\
$B$ & $p \supset \square \diamond p$ \\
\hline
\end{tabular}

Tabela 2.4: Axiomas modais e respectivas sentenças

Os modelos de lógicas modais normais serão representados pela letra $\mathcal{M}$. Seja $A$ o conjunto de átomos da linguagem. Para [Che80], um modelo $\mathcal{M}$ de uma lógica modal normal (chamado de modelo de Kripke) é uma tripla $\langle W, R, V\rangle$ em que

- $W$ é um conjunto (de mundos possíveis)

- $R$ é uma relação binária sobre $M: R \subset M \times M$, chamada de relação de acessibilidade

- $V$ é uma função de $W \times A$ em $\{0,1\}$, isto é, associa a cada par $\left(w, p_{i}\right) \circ$ valor 0 ou 1

A função $V$ é chamada, como na Seção 2.1.1, de valoração. Intuitivamente, $W$ é uma coleção de "mundos" para os quais as sentenças do sistema formal significarão algo, sendo ou verdadeiras ou falsas.

Agora, serão especificadas recursivamente sobre que condições uma sentença pode ser aceita como verdadeira ou falsa para cada elemento de $W$, ou seja, para os mundos. Aqui, a expressão $\mathcal{M}, w \models \phi$ é uma abreviação para " $\phi$ é verdadeira em um mundo $w \in W^{\prime \prime}$; escreve-se $\mathcal{M}, w \not \forall \phi$ quando não for verdade que $\mathcal{M}, w \models \phi$. Tal definição é encontrada em [Che80, HC96].

\footnotetext{
${ }^{3} \mathrm{Em}$ geral, neste texto segue-se o sistema de nomenclatura de [Che80], em que o nome de uma lógica é dado pela listagem dos axiomas modais que nela aparecem. Uma lógica que tenha por axiomas $K$ e 4, por exemplo, é designada por K4. Exceções a essa regra ocorrem apenas nos casos em que o nome do sistema é consagrado pelo uso, como nos casos dos sistemas T e D.
} 


\section{Definição Recursiva de Verdade em Sentenças da Lógica Modal}

NORMAL

1. $\mathcal{M}, w \models p_{i}$ sse $V(w)\left(p_{i}\right)=1$

2. $\mathcal{M}, w \models \neg \phi$ sse $\mathcal{M}, w \not \models \phi$

3. $\mathcal{M}, w \models \phi \wedge \psi$ sse $\mathcal{M}, w \models \phi$ e $\mathcal{M}, w \models \psi$

4. $\mathcal{M}, w \models \phi \vee \psi$ sse $\mathcal{M}, w \models \phi$ ou $\mathcal{M}, w \models \psi$

5. $\mathcal{M}, w \models \phi \supset \psi$ sse ou $\mathcal{M}, w \not \models \phi$ ou $\mathcal{M}, w \models \psi$

6. $\mathcal{M}, w \models \phi \equiv \psi$ sse, se $\mathcal{M}, w \models \phi$, então $\mathcal{M}, w \models \psi$, e vice-versa

7. $\mathcal{M}, w \models \square \phi$ sse para todo $t \in W$ tal que $w R t$ tem-se $\mathcal{M}, w \models \square \phi$

8. $\mathcal{M}, w \models \diamond \phi$ sse existe $t \in W$ tal que $w R t$ e $\mathcal{M}, w \models \square \phi$

A cláusula 1 dessa definição recursiva dá uma intuição sobre o que é $V$ : tem-se que $V(w)(i)=1$ justamente quando $p_{i}$ é verdadeira em $w$.

$\mathrm{O}$ conjunto de mundos $t$ para os quais $w R t$, em que $w$ é um mundo, podem ser pensados intuitivamente como o conjunto de mundos acessiveis a $w$. A maneira de enxergar os diversos mundos ficará mais clara adiante, quando for descrito um contra-modelo de um dos princípios sintáticos das Lógicas Modais.

A relação entre sintaxe e semântica de certa lógica $\mathrm{L}$ é feita demonstrandose correção e completude. A forma de demonstrar essas propriedades acaba passando pela construção de determinados modelos. Mas cada valoração dá origem a um modelo diferente e cada relação de acessibilidade dá origem a toda uma classe de modelos distintos. Torna-se necessário, portanto, definir graus de validade das sentenças.

Certas definições adicionais são encontradas em [Gor99]. Uma estrutura de Kripke é um par $\langle W, R\rangle$. Cada modelo de Kripke origina-se a partir de uma estrutura. Uma classe de estruturas de Kripke é uma conjunto de estruturas de Kripke com o mesmo conjunto $W$ e com relações de acessibilidade que possuem certas propriedades comuns (por exemplo, relações que são simétricas).

Uma sentença $\phi$ é válida em um modelo de Kripke $\mathcal{M}$ (denota-se esse fato por $\mathcal{M} \models \phi$ ) quando, para todo $w \in W$, ocorrer $\mathcal{M}, w \models \phi$. Uma sentença $\phi$ é válida em uma estrutura de Kripke $\langle W, R\rangle$ (denota-se esse fato por $\langle W, R\rangle \models \phi$ ) quando, para todo modelo $\mathcal{M}$ que tem $\langle W, R\rangle$ por estrutura, ocorrer $\mathcal{M} \models \phi$. Por fim, uma sentença $\phi$ é válida em uma classe de estruturas de Kripke $\mathcal{C}$ (denota-se esse fato por $\mathcal{C} \vDash \phi$ ) quando, para toda estrutura $\langle W, R\rangle$ em $\mathcal{C}$, ocorrer $\langle W, R\rangle \models \phi$.

A noção de conseqüência lógica precisa ser refinada, e a apresentada aqui é devida a Massacci [Mas98a]. Sejam $\Gamma_{U}$ e $\Gamma_{G}$ dois conjuntos finitos de sentenças. A sentença $\psi$ é conseqüência lógica local do conjunto de hipóteses locais $\Gamma_{U}$ (denota-se $\Gamma_{U} \models \psi$ ) quando para todo modelo $\mathcal{M}$ e todo mundo $w \in W$, se $\mathcal{M}, w \models \Gamma_{U}{ }^{4}$, então $\mathcal{M}, w \models \psi$. A sentença $\psi$ é conseqüência lógica global do

${ }^{4} \mathrm{Ou}$ seja, $\mathcal{M}, w \models \phi$ para cada sentença $\phi \in \Gamma_{U}$. 
conjunto de hipóteses globais $\Gamma_{G}$ (denota-se $\Gamma_{G} \models \psi$ ) quando para todo modelo $\mathcal{M}$ e todo mundo $w \in W$, se $\mathcal{M} \models \Gamma_{G}$, então $\mathcal{M}, w \models \psi$. Toda conseqüência lógica global é local, mas a recíproca não é verdadeira. Para ambas as noções de conseqüência lógica, vale o teorema da dedução: dada uma classe de estruturas $\mathcal{C}$ e $\Gamma_{U \mid G}=\left\{\phi_{1}, \phi_{2}, \ldots, \phi_{n}\right\}, \Gamma_{U \mid G} \models \psi$ sse $\mathcal{C} \models \phi_{1} \wedge \phi_{2} \wedge \cdots \wedge \phi_{n} \supset \psi$. Suponha que sejam dados o conjunto de hipóteses locais $\Gamma_{U}$ e globais $\Gamma_{G}$. Para todo modelo $\mathcal{M}$ e todo mundo $w \in W$, se $\mathcal{M} \models \Gamma_{G}$ e $\mathcal{M}, w \models \Gamma_{U}$ implicarem que $\mathcal{M}, w \models \psi$, então $\psi$ é conseqüência lógica de hipóteses globais $\Gamma_{G}$ e locais $\Gamma_{U}$, $\mathrm{e}$ utiliza-se a notação $\Gamma_{G} \models \Gamma_{U} \Rightarrow \psi^{5}$.

Os diferentes princípios modais dados na Tabela 2.4 podem ou não ser válidos em determinadas classes de estruturas de Kripke; de fato, para cada um dos princípios apresentados na Tabela 2.4, é possível dizer exatamente qual a classe de estruturas em que eles valem, no sentido que, se uma estrutura não é dessa classe, então o princípio não vale em pelo menos um modelo originado a partir dela. O único princípio que é válido em qualquer classe é $K$.

Em uma dada lógica modal $\mathbf{L}$, a correção significa que não é possível provar um par de teoremas $\phi$ e $\neg \phi$ a partir da mesma. Da própria definição de verdade de sentenças $\neg \phi$, em um modelo nunca ocorre simultaneamente $\phi$ e $\neg \phi$. Assim, a estratégia para provar correção é mostrar que os axiomas de $\mathbf{L}$ são válidos em algum modelo $\mathcal{M}$ e mostrar que, se uma sentença é obtida de outras (supostas válidas em $\mathcal{M}$ ) por aplicação de uma regra de inferência, então ela mesma é válida em $\mathcal{M}$. Assim, se fosse possível provar $\phi$ e $\neg \phi$, ambas seriam válidas no modelo $\mathcal{M}$, o que é absurdo. A completude de $\mathbf{L}$ significa que, dada uma sentença $\phi$ qualquer, se $\phi$ não for um teorema, sua adição ao sistema implica em uma contradição. Em cada modelo, é necessariamente válido que ou $\phi$ ou $\neg \phi$ ocorram (se $\phi$ não acontece, a definição de verdade para negações implica que $\neg \phi$ acontece); se $\phi$ não for verdadeira para o modelo, ela não pode ser adicionada porque isso resultaria em contradição - uma vez que $\neg \phi$ já é verdadeira no modelo. Portanto, a estratégia para mostrar completude é construir um modelo $\mathcal{M}$ em que todos os axiomas de $\mathbf{L}$ sejam válidos, bem como provar que a aplicação de regras de inferência sobre sentenças válidas leva a outras sentenças válidas. Entretanto, para se ter certeza de que nenhum outro fato sobre o modelo particular $\mathcal{M}$ seja utilizado (por exemplo, a valoração $V$ e a relação de acessibilidade $R$ específicas) o modelo deve ser muito cuidadosamente construído. Como observado acima, os diferentes princípios modais - e, a fortiori, os diversos sistemas de lógicas modais obtidas por combinação dos princípios - não são válidos em qualquer classe de estruturas, mas apenas em uma. O modelo construído para mostrar completude deve ser tal que não priorize nenhuma propriedade que não seja válida em todos os modelos originados a partir de qualquer estrutura da classe de estruturas em que o princípio vale. Isso acaba dando origem ao método dos modelos canônicos, apresentado em [Che80, HC96].

\footnotetext{
${ }^{5}$ Observa-se que o símbolo $\Rightarrow$ utilizado nesta expressão não é um símbolo da linguagem, $\mathrm{e}$ sim da metalinguagem.
} 
Dessa forma, as definições de correção e completude são mais convenientemente apresentadas como a seguir, em que é utilizada a noção de classes de estruturas.

Seja $\mathcal{C}$ uma classe de estruturas de Kripke e $\mathbf{L}$ uma lógica modal. Segundo [Gor99], L é correta com relação a $\mathcal{C}$ quando toda sentença $\phi$ que é demonstrável em $\mathbf{L}\left(\vdash_{\mathbf{L}} \phi\right)$ for válida em $\mathcal{C}(\mathcal{C} \models \phi)$, e $\mathbf{L}$ é completa com relação a $\mathcal{C}$ quando toda sentença que é válida em $\mathcal{C}(\mathcal{C} \models \phi)$ for demonstrável em $\mathbf{L}\left(\vdash_{\mathbf{L}} \phi\right)$. Uma lógica $\mathbf{L}$ é caracterizada por $\mathcal{C}$ quando $\mathbf{L}$ for correta e completa com relação a C.

Um dos problemas da Lógica Modal, já observado, é caracterizar que relações de acessibilidade $R$ (ou seja, que classes de estruturas definidas por $R$ ) correspondem aos vários princípios sintáticos da Lógica Modal, como por exemplo $D$, $T, B, 4$ e 5 . Provando correção e completude de lógicas que utilizem apenas cada um desses axiomas, é possível chegar à caracterização dos princípios conforme apresentada na Tabela 2.5 (extraída de [Gor99]), em que são dadas as condições sobre a relação de acessibilidade das classes de estruturas caracterizadoras, bem como as sentenças de primeira ordem que descrevem essas condições.

\begin{tabular}{|c|c|c|}
\hline Axioma & Condição & Sentença de primeira ordem \\
\hline$T$ & Reflexiva & $\forall w(w R w)$ \\
$D$ & Serial & $\forall w \exists t(w R t)$ \\
4 & Transitiva & $\forall w \forall t \forall v(w R t \wedge t R v \supset w R v)$ \\
5 & Euclidiana & $\forall w \forall t \forall v(w R t \wedge w R v \supset t R v)$ \\
$B$ & Simétrica & $\forall w \forall t(w R t \supset t R w)$ \\
\hline
\end{tabular}

Tabela 2.5: Caracterização dos axiomas modais

Para a demonstração de que princípios como $D, T, B, 4$ e 5 não são válidos para todos os modelos de Kripke, torna-se necessário encontrar contra-modelos para cada um deles.

Por exemplo: para invalidar 4, é necessário encontrar um modelo que invalide uma instância (ou seja, uma sentença obtida por substituição de $p$ ) desse princípio. O contra-modelo a seguir é tirado de [Che80].

Seja $\mathcal{M}=\langle W, R, V\rangle$, em que

- $W=\{w, t, v\}$ (todos distintos)

- $R=\{(w, t),(t, v)\}$

- $V(t)\left(p_{i}\right)=1$ para todo $i \in \mathbb{N}$ e $V=0$ nos outros casos

Dessa forma, $\mathcal{M}, t \models p_{0}$ mas não $\mathcal{M}, v \models p_{0}$. Como $v$ é acessivel a $t$, isso implica que não $\mathcal{M}, t \models \square p_{0}$, ou seja, que $\mathcal{M}, t \models \neg \square p_{0}$. Mas $t$ é acessível a $w$. Logo não pode ocorrer $\mathcal{M}, t \models \square \square p_{0}$, o que quer dizer que $\mathcal{M}, t \models \neg \square \square p_{0}$. Por outro lado, para o único mundo acessível a $w$, que é $t$, vale $p_{0}$, e isso pode ser escrito como $\mathcal{M}, w \models \square p_{0}$. Conclusão: não ocorre $\mathcal{M}, w \models \square p_{0} \supset \square \square p_{0}$, o que invalida 4. 
Na Figura 2.2, adaptada de [Che80], é possível visualizar essa prova. Os pontos representam os mundos e embaixo desses pontos ficam algumas sentenças válidas nesses mundos. As flechas de um mundo a outro indicam que o mundo para onde a flecha vai é acessível ao mundo de onde a flecha sai.

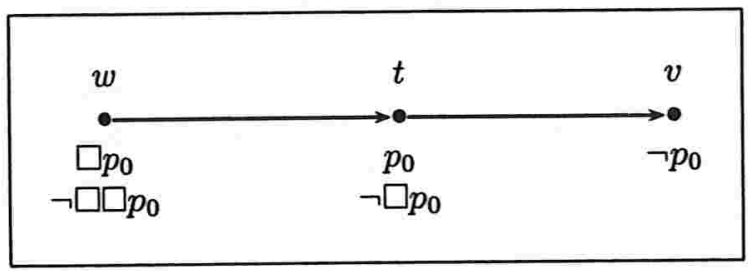

Figura 2.2: Invalidação do princípio modal 4

Diferentes sistemas de lógicas modais são obtidos pela combinação dos vários princípios. Como cada princípio é caracterizado por certa classe de estruturas, a combinação dos diversos princípios é caracterizada pela intersecção das respectivas classes de estruturas. Na Tabela 2.6 (extraída de [Gor99]), são apresentados os sistemas que serão utilizados neste texto e suas caracterizações.

\begin{tabular}{|c|c|c|}
\hline Lógica & Axiomas & Classes de estruturas de Kripke \\
\hline $\mathbf{K}$ & $K$ & Todas \\
$\mathbf{T}$ & $K T$ & Reflexivas \\
$\mathbf{D}$ & $K D$ & Seriais \\
K4 & $K 4$ & Transitivas \\
K5 & $K 5$ & Euclidianas \\
KB & $K B$ & Simétricas \\
KDB & $K D B$ & Simétricas e seriais \\
$\mathbf{B}$ & $K T B$ & Reflexivas e simétricas \\
KD4 & $K D 4$ & Seriais e transitivas \\
K45 & $K 45$ & Transitivas e euclidianas \\
KD5 & $K D 5$ & Seriais e euclidianas \\
KD45 & $K D 45$ & Seriais, transitivas e euclidianas \\
S4 & $K T 4$ & Reflexivas e transitivas \\
KB4 & $K B 4$ & Simétricas e transitivas \\
S5 & $K T 5$ & Reflexivas, transitivas e simétricas \\
\hline
\end{tabular}

Tabela 2.6: Lógicas modais e respectivas caracterizações

\subsubsection{Tableaux de Prova}

Os tableaux modais de prova utilizados neste texto são uma variante dos Tableaux Prefixados de Fitting [Fit83]. Essa variante, chamada SST (de "Single Step Tableaux", tableaux passo a passo), foi desenvolvida por Fabio Massacci [Mas98c, Mas00] e Rajeev Goré [Gor99]. Entre as vantagens enumeradas por Massacci [Mas00] para os SST, estão a modularidade do método (ou seja, a 
adição de regras aumenta o poder de demonstração da lógica), propriedades computacionais atraentes e a capacidade de descrever diversas lógicas modais básicas.

Nos SST, antes de toda sentença assinalada $\varphi$ coloca-se um prefixo $\sigma$ (denotase essa operação por $\sigma: \varphi$ ). Esse prefixo é uma seqüência de números inteiros positivos; o primeiro elemento da seqüência é sempre 1. Exemplo de prefixo: $\sigma=1.4 .2 .2$. Intuitivamente, $\sigma$ é o nome de um mundo em que $\varphi$ é satisfeita. A passagem de um prefixo $\sigma$ para outro prefixo $\sigma . m$ pode ser compreendida como sendo a realização de um passo introspectivo. Essa representação é útil porque codifica todos os passos de introspecção desde o mundo "real", denotado por 1 , até o mundo $\sigma$. O comprimento do prefixo $\sigma$, denotado $|\sigma|$, é o número de passos de introspeçãa, ou seja, $|1|=0$ e $\left|1 . m_{1} \ldots \ldots m_{k}\right|=k$. Assim, $|1.4 .2 .2|=3$.

Massacci observa, em [Mas98a], que a escolha de procedimentos dedutivos para lógicas modais é quase tão grande quanto o número de lógicas modais. Isso acaba implicando em diversas regras, que poderão ou não ser usadas dependendo do sistema de lógica modal em que se está trabalhando. Nos SST, existem regras que aparecem em todas as lógicas - em particular, as regras que dizem como lidar com os conectivos lógicos clássicos -, enquanto há outras regras que podem variar de uma lógica a outra. Essas últimas regras são sempre sobre os conectivos modais. A aplicação de uma regra $R$ qualquer a uma sentença prefixada $\sigma: \varphi$ fornece uma outra sentença prefixada, que será denotada $\sigma^{*}: \varphi^{R}$.

Fitting [Fit83] distinguiu dois tipos de sentenças que utilizam conectivos modais. Considere a sentença assinalada $T \square \phi$. Aceitá-la como verdadeira em um mundo $w$ implica na aceitação de que, para todo um mundo possível $t$ acessível de $w$, necessariamente $T \phi$ é verdadeira. Da mesma forma, a aceitação de $F \diamond \phi$ em um mundo $w$ implica na aceitação necessária de $F \phi$ em todo mundo acessível de $w$. Sentenças desse tipo foram chamadas por Fitting de sentenças $\nu$, de "necessidade". Considere agora a sentença assinalada $F \square \phi$. Aceitá-la como verdadeira em um mundo $w$ implica na aceitar a possibilidade de existir pelo menos um mundo possível $t$ acessível de $w$ em que $F \phi$ seja verdadeira. Da mesma forma, a aceitação de $T \diamond \phi$ em um mundo $w$ implica na aceitação de que existe pelo menos um mundo acessível de $w$ em que $T \phi$ aconteça. Sentenças desse tipo foram chamadas por Fitting de sentenças $\pi$, de "possibilidade". A Tabela 2.7 sumariza a notação unificadora de Fitting.

\begin{tabular}{|cc|}
\hline$\nu$ & $\nu_{0}$ \\
\hline$T \square \phi$ & $T \phi$ \\
$F \diamond \phi$ & $F \phi$ \\
\hline
\end{tabular}$\quad$\begin{tabular}{cc}
\hline$\pi$ & $\pi_{0}$ \\
\hline$F \triangleright \phi$ & $F \phi$ \\
$T \diamond \phi$ & $T \phi$ \\
\hline
\end{tabular}

Tabela 2.7: Notação unificadora de Fitting

A demonstração de uma sentença não assinalada $\phi$ é feita como segue. Assinala-se $\phi$ como $F \phi$; prefixa-se $F \phi$ como $1: F \phi$. A seguir, tenta-se construir o Tableau SST para $\{1: F \phi\}$. Basicamente, isso significa que será tentada uma refutação da sentença $\phi$ no mundo "real" 1 . Se o tableau fecha ${ }^{6}$, a sentença foi

${ }^{6}$ Isto é, se aparece no tableau uma sentença prefixada e sua conjugada; a conjugada de 
demonstrada; senão, a prova falhou. Para provar que, de um conjunto finito $\Gamma$ de sentenças não assinaladas pode-se inferir uma outra sentença (também não assinalada) $\psi$, primeiro assinala-se a sentença $\psi$ como $F \psi$. A seguir, deve ser feita uma distinção entre as sentenças de $\Gamma$. As sentenças que são válidas em todos os mundos são chamadas de hipóteses globais, contidas em $\Gamma_{G} \subseteq \Gamma$. As sentenças que são válidas apenas no mundo 1 são chamadas de hipóteses locais, contidas em $\Gamma_{U} \subseteq \Gamma$. Assim, $\Gamma_{G} \cup \Gamma_{U}=\Gamma$ e $\Gamma_{G} \cap \Gamma_{U}=\emptyset$. Em cada caso, deve ser dado se uma determinada sentença em $\Gamma$ é uma hipótese global ou local. Sentenças $\phi_{G} \in \Gamma_{G}$ devem ser assinaladas como falsas $-F \phi_{G}-$ e podem ser introduzidas em qualquer ramo do tableau após qualquer prefixo $-\sigma: F \phi_{G}$. Essa regra é chamada de regra global, Glob. Sentenças $\phi_{U} \in \Gamma_{U}$ devem ser assinaladas como verdadeiras $-F \phi_{U}-$ e podem ser introduzidas em qualquer ramo do tableau apenas após o prefixo $1-1: F \phi_{U}$. Essa regra é chamada de regra local, Loc. Se o tableau fechar, o seqüente $\Gamma \vdash \psi$ foi provado.

Os SST possuem as seguintes regras:

T1. para toda sentença prefixada da forma $\sigma: \alpha$, adicionar $\sigma: \alpha_{1}$ e $\sigma: \alpha_{2}$ ao ramo do tableau

T2. para toda sentença da forma $\sigma: n e g$, adicionar $\sigma:$ pos ao ramo do tableau

T3. para toda sentença assinalada da forma $\beta$, ramificar em dois o tableau, adicionando $\sigma: \beta_{1}$ a um ramo e $\sigma: \beta_{2}$ ao outro ramo do tableau

T4. para toda sentença da forma $\sigma: \pi$, adicionar $\sigma . m: \pi_{0}$ ao ramo do tableau ( $m$ pode ser qualquer número inteiro positivo, desde que o prefixo $\sigma . m$ não exista previamente no ramo)

T5. para toda sentença da forma $\sigma: \varphi$ e outras possíveis regras $R$ (que variam de lógica a lógica), adicionar $\sigma^{*}: \varphi^{R}$ ao ramo do tableau para todo prefixo $\sigma^{*}$ presente no ramo

As regras dos SST para os conectivos proposicionais são sumarizadas nas Tabela 2.8 .

$$
\begin{array}{lll}
\frac{\sigma: \alpha}{\sigma: \alpha_{1}} & \frac{\sigma: n e g}{\sigma: p o s} & \frac{\sigma: \beta}{\sigma: \beta_{1} \quad \sigma: \beta_{2}} \\
\sigma: \alpha_{2} &
\end{array}
$$

Tabela 2.8: Regras proposicionais para SST

As outras regras SST que valem para todas as lógicas são dadas na Tabela 2.9 , tirada de [Mas98a]; a regra $(\pi)$ enunciada é a única que permite a criação de novos prefixos nos ramos do tableau. Todas as sentenças que aparecem após o prefixo são assinaladas.

uma sentença prefixada $\sigma: \varphi$ é $\sigma: \bar{\varphi}$. Tal observação é evidente, mas precisa ser feita por razões de completude. 


$$
\begin{array}{ll}
\frac{\vdots}{1: \varphi}(\text { Loc }) & \text { se } \varphi \in \Gamma_{U} \\
\frac{\vdots}{\sigma: \varphi}(G l o b) & \text { se } \sigma \text { já existir no ramo e } \varphi \in \Gamma_{G} \\
\frac{\sigma: \pi}{\sigma . m: \pi_{0}}(\pi) & \text { se } \sigma . m \text { for novo no ramo }
\end{array}
$$

Tabela 2.9: Regras modais para SST válidas em todas as lógicas

As regras SST que variam de lógica a lógica e as caracterizam (ao menos para as lógicas modais básicas) são dadas na Tabela 2.10, tirada de [Mas98a]. Algumas observações sobre essa tabela. Só se podem aplicar regras quando todos os prefixos envolvidos já apareceram no ramo. Na regra $(D)$, se o antecedente for $\nu=T \square \phi$, então $\nu_{o p}=T \diamond \phi$; se for $\nu=F \diamond \phi$, então $\nu_{o p}=F \square \phi$. Na regra $(C x t)$, se o antecedente for $\nu=T \square \phi$, então $\nu^{2}=T \square \square \phi$, e similarmente para $F \diamond \phi$.

$$
\begin{array}{ccc}
\frac{\sigma: \nu}{\sigma . m: \nu_{0}}(K) & \frac{\sigma: \nu}{\sigma: \nu_{o p}}(D) & \frac{\sigma: \nu}{\sigma: \nu_{0}}(T) \\
\frac{\sigma: \nu}{\sigma . m: \nu}{ }_{(4)} & \frac{\sigma \cdot m: \nu}{\sigma: \nu}{ }_{\left(4^{R}\right)} & \frac{\sigma \cdot m: \nu}{\sigma: \nu_{0}}(B) \\
\frac{\sigma . m: \pi}{\sigma: \pi}\left(4^{\pi}\right) & \frac{\sigma \cdot m: \nu}{\sigma \cdot m \cdot l: \nu}{ }_{\left(4^{D}\right)} & \frac{\sigma \cdot m: \nu}{\sigma: \nu^{2}}(C x t)
\end{array}
$$

$\sigma, \sigma . m$ e $\sigma . m . l$ já devem estar no ramo do tableau

Tabela 2.10: Regras modais para SST que caracterizam lógicas

Na Tabela 2.11, extraída de [Mas00], são dadas as regras SST que caracterizam cada lógica modal básica. As regras que aparecem entre colchetes são deriváveis das outras que descrevem a lógica em questão. Assim, a regra $(K)$ pode ser inferida utilizando (4) e $(T)$ :

$$
\frac{\sigma: \nu}{\frac{\sigma . m: \nu}{\sigma . m: \nu_{0}} \operatorname{de}(4)} \operatorname{de}(T)
$$

Algumas lógicas podem ser caracterizadas por mais de um conjunto de regras, como é o caso de S5.

O conceito de sentença reduzida não varia muito com relação ao dado na Seção 2.1.1. É necessário apenas adicionar os prefixos a todas as sentenças assinaladas nas regras para sentenças atômicas e dos tipo $\alpha$, neg e $\beta$, e adicionar 


\begin{tabular}{|c|c|c|}
\hline Lógica & Axiomas & Regras SST \\
\hline K & $K$ & $(K)$ \\
T & $K T$ & $(K)+(T)$ \\
D & $K D$ & $(K)+(D)$ \\
K4 & $K 4$ & $(K)+(4)$ \\
K5 & $K 5$ & $(K)+\left(4^{D}\right)+\left(4^{R}\right)+(C x t)$ \\
KB & $K B$ & $(K)+(B)$ \\
KDB & $K D B$ & $(K)+(B)+(D)$ \\
B & $K T B$ & $(K)+(T)+(B)$ \\
KD4 & $K D 4$ & $(K)+(D)+(4)$ \\
K45 & $K 45$ & $(K)+(4)+\left(4^{R}\right)$ ou \\
& & $(K)+\left(4^{\pi}\right)+\left(4^{R}\right)$ \\
KD5 & $K D 5$ & $(K)+(D)+\left(4^{D}\right)+\left(4^{R}\right)+(C x t)$ \\
KD45 & $K D 45$ & $(K)+(D)+(4)+\left(4^{R}\right)$ ou \\
& & $(K)+(D)+\left(4^{\pi}\right)+\left(4^{R}\right)$ \\
S4 & $K T 4$ & {$[(K)]+(T)+(4)$} \\
KB4 & $K B 4$ & $(K)+(B)+\left(4^{\pi}\right)+\left(4^{R}\right)$ \\
S5 & $K T 5$ & {$[(K)]+(T)+(4)+\left(4^{R}\right)$ ou } \\
& & $(K)+(T)+\left(4^{\pi}\right)+\left(4^{R}\right)$ \\
\hline
\end{tabular}

Tabela 2.11: Lógicas modais e respectivas regras SST

cláusulas para as sentenças modais. Uma sentença prefixada $\sigma: \varphi$ é reduzida em um ramo $R$ (segundo o SST) se

R1. $\sigma: \varphi$ é um átomo prefixado, ou seja, $\sigma: T p$ ou $\sigma: F p$ para algum átomo $p$

R2. $\sigma: \varphi$ é uma sentença $\sigma: \alpha$ e tanto $\sigma: \alpha_{1}$ como $\sigma: \alpha_{2}$ estão em $R$

R3. $\sigma: \varphi$ é uma sentença $\sigma:$ neg e $\sigma:$ pos está em $R$

R4. $\sigma: \varphi$ é uma sentença $\sigma: \beta$ e ou $\sigma: \beta_{1}$ ou $\sigma: \beta_{2}$ está em $R$

R5. $\sigma: \varphi$ é uma sentença $\sigma: \pi$ e $\sigma . m: \pi_{0}$ está em $R$ ( $\sigma . m$ não está previamente no ramo)

R6. $\sigma: \varphi$ é uma sentença e, para todas as outras possíveis regras $R$ (que variam de lógica a lógica) e todo prefixo $\sigma^{*}$ em $R, \sigma^{*}: \varphi^{R}$ está em $R$

Na Figura 2.3 é apresentado um exemplo de aplicação dos SST, extraído de [Gor99]. No exemplo, demonstra-se o princípio modal $K: \square(p \supset q) \supset(\square p \supset$ $\square q$ ); esse princípio vale em todas as lógicas e, além das regras SST válidas em qualquer sistema, é utilizada apenas a regra $(K)$. As sentenças (2)-(3) são obtidas de (1) pela regra $\alpha$. As sentenças (4)-(5), de (3) por $\alpha$ também. A sentença (6) é obtida de (5) por aplicação da regra $(\pi)$. As sentenças (7) e (8), de (2) e (4), respectivamente, por aplicação da regra $(K)$. Observa-se que essa regra só pôde ser aplicada porque o prefixo 1.1 já existia no (único) ramo do tableau. Por fim, as sentenças (9)-(10) são obtidas de (7) por $\beta$. 


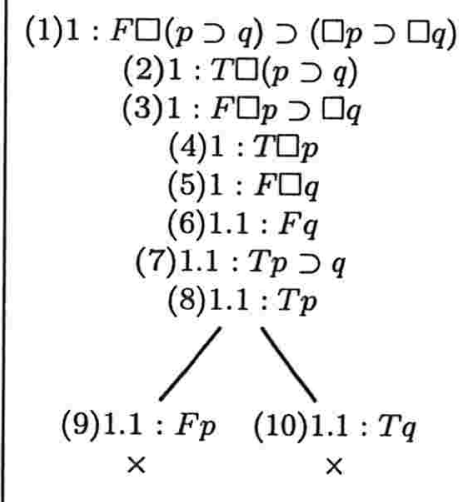

Figura 2.3: Um exemplo de aplicação dos SST

Seja $\Gamma$ um conjunto finito de sentenças não assinaladas. Lembrando da definição dada na Seção 2.1.2, demonstrar a correção do tableau significa mostrar que, se um tableau para o seqüente $\Gamma \vdash \psi$ fecha, então $\Gamma$ implica logicamente $\psi$. É suficiente mostrar que a aplicação das regras do tableau leva de sentenças satisfatíveis a outras sentenças satisfatíveis. A diferença agora é que a noção de modelo precisa ser estendida, levando em consideração os prefixos. Intuitivamente, os prefixos designam mundos possíveis (na designação, são codificados todos os passos introspectivos necessários para se chegar ao mundo possível designado). O SST de uma dada sentença pode ser pensado como uma tentativa sistemática de construir um contra-modelo para a mesma, invalidando-a no mundo designado pelo prefixo 1 . Cada uma das regras expressa as conseqüências de se aceitarem certas hipóteses. Como os prefixos devem designar mundos, e cada lógica é caracterizada por uma relação de acessibilidade, torna-se necessário construir uma álgebra de prefixos, a partir da qual é possível determinar que prefixo é acessível de que prefixo. Além disso, deve-se impor que o mundo designado pelo prefixo $\sigma . m$ é acessível ao mundo designado pelo prefixo $\sigma$. Cada conjunto diferente de regras para lógicas vem a resultar numa álgebra distinta de prefixos, de tal forma que a identificação de prefixos com mundos permita simular a relação de acessibilidade entre mundos a partir da acessibilidade entre os prefixos. A natureza das regras é "passo a passo" - daí o nome do método -, ou seja, elas só dizem como prefixos $\sigma$ se relacionam com prefixos no máximo um passo adiante (prefixos $\sigma . m$ ) ou vice-versa. Portanto, a simulação da relação de acessibilidade é obtida por aplicações múltiplas das regras de acessibilidade entre os prefixos. Os detalhes da prova, apresentados em [Mas00, Gor99], dão como resultado a

Proposição 2.4 (Correção) Suponha que um tableau para $\Gamma_{U} \cup \Gamma_{G} \vdash \psi$ feche, em que $\Gamma_{U}$ é o conjunto de hipóteses locais e $\Gamma_{G}$ o conjunto de hipóteses globais. Então $\Gamma_{G} \models \Gamma_{U} \Rightarrow \psi$. 
Mostrar a completude para tableaux é mostrar que, se um tableau não fecha em um ramo, então o ramo que não fecha é satisfatível por um modelo. Um ramo aberto dá indicações de como construir um contra-modelo da sentença que se desejava provar. Os mundos possíveis, nesse caso, podem ser os próprios prefixos; a álgebra de prefixos é utilizada para construir a relação de acessibilidade entre os mesmos. Cada sistema modal é caracterizado por uma relação de acessibilidade e, portanto, por uma álgebra de prefixos. Os detalhes da prova são apresentados em [Mas00, Gor99], resultando na

Proposição 2.5 (Completude) Suponha que $\Gamma_{G} \vDash \Gamma_{U} \Rightarrow \psi$, em que $\Gamma_{U}$ é $o$ conjunto de hipóteses locais e $\Gamma_{G}$ o conjunto de hipóteses globais. Então o tableau para $\Gamma_{U} \cup \Gamma_{G} \vdash \psi$ fecha.

Por causa da natureza de algumas das regras SST, que permitem reutilizar sentenças tão logo apareça um novo prefixo, pode ocorrer (e de fato ocorre) a existência de ramos infinitos em tableaux, no caso da prova falhar (se a prova funciona, o tableau é necessariamente finito). Massacci [Mas00] apresenta técnicas que permitem a verificação de que determinado ramo, se reduzido, se tornará infinito (ele chama a técnica de "checagem de loops"); naturalmente, nesse caso a prova falhou, mas a técnica possibilita também um controle sobre a geração do contra-modelo.

Por causa da existência das diversas regras para os SST, que variam de lógica a lógica, existem diversas regras de simplificação e não apenas uma, como no caso proposicional. Em [Mas98a], Massacci explica como incorporar simplificação nos SST. 


\section{Capítulo 3}

\section{O Método de Cadoli-Schaerf}

Em [SC95], Marco Cadoli e Marco Schaerf fazem uma comparação entre os métodos de Levesque e de Selman e Kautz, apresentados no Capítulo 1. Entre os fatos observados, eles apontam que o método de Levesque, caracterizado por possuir motivações filosóficas, admite apenas inferências corretas, ao passo que o método de Selman e Kautz, com motivações principalmente computacionais, admitem inferências corretas e inferências completas. Eles então apresentam uma série de guias para um novo método de aproximação (extraído de [SC95]):

bem-fundamentado semanticamente: as respostas aproximadas devem dar informações semanticamente claras ao problema em questão

computacionalmente atraente: as respostas aproximadas devem ser mais fáceis de calcular que as respostas ao problema original

incrementabilidade: as respostas aproximadas podem ser incrementadas, e eventualmente convergem à resposta correta, desde que se tenha tempo e motivação (ou capacidade) suficientes

dual: tanto aproximações corretas como aproximações completas devem ser descritas

flexível: o esquema de aproximação deve ser suficientemente geral para ser aplicável a uma grande variedade de problemas de raciocínio

Em seu artigo [SC95], Cadoli e Schaerf afirmam ter adicionado ao método de Levesque dualidade e incrementabilidade, mantendo suas boas características computacionais e seu sabor semântico vindo de uma formulação clara em termos de lógicas multivaloradas. 


\subsection{Semântica e Principais Propriedades}

Seja $A$ o conjunto dos átomos da linguagem e $S \subseteq A$ um subconjunto - possivelmente não próprio - $\operatorname{de} A$.

Definição 3.1 Uma $S_{3}$-interpretação de $A$ é uma valoração total no conjunto dos literais $A^{\circ}$ que leva cada par de literais em $S^{\circ}$ em valores opostos - isto é, $V(p)=0$ sse $V(\neg p)=1$, para um átomo $p \in S$. Além disso, ela não leva ao mesmo tempo um par de literais em $(A \backslash S)^{\circ}$ a $0-$ ou seja, nunca ocorre $V(p)=V(\neg p)=0$, para um átomo $p \in A$.

Assim, literais em $S^{\circ}$ se comportam classicamente. Por outro lado, literais em $(A \backslash S)^{\circ}$ podem se comportar paraconsistentemente. A intuição por trás de todos os argumentos pode ser descrita da seguinte maneira: sentenças cujos átomos estejam em $S$ representam um conhecimento acessível ao agente pensante. Nesse contexto, costuma-se usar a expressão "proposições relevantes" para o conjunto $S$. Fora de $S$, o conhecimento é inacessível ao agente pensante, e podem ocorrer contradições (um par de literais opostos que são levados ao mesmo valor) sem que isso prejudique o raciocínio sobre as proposições relevantes. Em um agente onisciente, qualquer contradição trivializa a lógica, no sentido que qualquer proposição é verdadeira.

De acordo com a Definição 3.1 , para cada átomo $p \in A \backslash S$ e cada $S_{3}$ interpretação $V$ há três possibilidades (sendo essa a razão do nome $S_{3}$-interpretação):

1. $V(p)=1, V(\neg p)=0$

2. $V(p)=0, V(\neg p)=1$

3. $V(p)=1, V(\neg p)=1$

Quando o conjunto $S=\emptyset$, a $S_{3}$-interpretação reduz-se à 3 -interpretação de Levesque; no caso $S=A$, ela reduz-se à 2-interpretação clássica (ver Definição 1.2). Dessa forma, generalizam-se as idéias de Levesque.

A associação de valores-verdade para outras sentenças é feita, para CadoliSchaerf, apenas para sentenças em forma normal negativa, $\mathrm{NNF}^{1}$, segundo as regras:

Regra $\vee: V(\phi \vee \psi)=1$ sse $V(\phi)=1$ ou $V(\psi)=1$

Regra $\wedge: V(\phi \wedge \psi)=1$ sse $V(\phi)=V(\psi)=1$

Como a $S_{3}$-interpretação generaliza tanto a 3-interpretação de Levesque quanto a 2-interpretação clássica, algumas regras de inferência, como modus ponens, podem ou não valer segundo o conjunto de proposições relevantes.

De maneira padrão, define-se $S_{3}$-inferência, representada por $\models_{S}^{3}$ :

${ }^{1}$ Ou seja, ocorrem apenas os operadores $\vee$ e $\wedge$, e negações ocorrem apenas nos átomos; toda sentença pode ser convertida em uma sentença NNF através de uma série de tranformações de equivalência com consumo linear de tempo e espaço. 
Definição 3.2 Define-se $\phi \models_{S}^{3} \psi$ sse toda $S_{3}$-interpretação que satisfaz $\phi$ satisfizer também $\psi$.

Dado um conjunto $\Gamma$ de sentenças, o significado de $\Gamma \models_{S}^{3} \phi$ é similar ao dado na Seção 2.1.1. Tais adaptações de notação, cujo significado é padrão, não serão apontadas neste texto.

A noção dual de $S_{1}$-interpretação é dada pela

Definição 3.3 Uma $S_{1}$-interpretação de L é uma valoração total no conjunto dos literais $A^{\circ}$ que leva cada par de literais conjugados em $S^{\circ}$ a valores opostos - isto é, $V(p)=0$ sse $V(\neg p)=1$, para um átomo $p \in S$. Além disso, ela leva ao mesmo tempo todo par de literais em $(A \backslash S)^{\circ}$ a 0 - ou seja, para um átomo $p \in A \backslash S$ sempre ocorre $V(p)=V(\neg p)=0$.

Para cada átomo $p \in A \backslash S$ e cada $S_{1}$-interpretação $V$ há apenas uma possibilidade (sendo essa a razão do nome $S_{1}$-interpretação):

$$
V(p)=0, V(\neg p)=0
$$

Quando o conjunto $S=A$, a $S_{1}$-interpretação reduz-se à 2-interpretação clássica. Define-se $S_{1}$-inferência da maneira costumeira, nos moldes da Definição 3.2 .

A intuição por trás dessa definição é que, fora do conjunto $S$ de proposições relevantes, o conhecimento é incorreto e não é possível tirar nenhuma conclusão. A ligação disso com a noção de $S_{3}$-aproximação e com a Lógica Clássica pode ser visualizada em termos de modelos. Seja $\Gamma$ um conjunto qualquer de sentenças em CNF. Lembrando da definição dada na Seção 2.1.1, um modelo para $\Gamma$ é qualquer valoração que satisfaça todas as sentenças de $\Gamma$. Quando se restringe a funções-verdade clássicas, obtêm-se modelos clássicos de $\Gamma$, designados por $\mathcal{M}_{C}(\Gamma)$. Quando se restringe a $S_{3}$-interpretações, obtêm-se modelos $S_{3}$, designados por $\mathcal{M}_{S_{3}}(\Gamma)$, e quando se restringe a $S_{1}$-interpretações, obtêm-se modelos $S_{1}$, designados por $\mathcal{M}_{S_{1}}(\Gamma)$. Vale (ver Teorema 3.4)

$$
\mathcal{M}_{S_{1}}(\Gamma) \subseteq \mathcal{M}_{C}(\Gamma) \subseteq \mathcal{M}_{S_{3}}(\Gamma)
$$

Ou seja, há mais modelos clássicos que modelos $S_{1}$, e mais modelos $S_{3}$ que modelos clássicos.

Na verdade, algo muito mais forte que isso vale. Se $S \subseteq S^{\prime}$, então ocorrem $\mathcal{M}_{S_{1}}(\Gamma) \subseteq \mathcal{M}_{S_{1}^{\prime}}(\Gamma)$ e $\mathcal{M}_{S_{3}^{\prime}}(\Gamma) \subseteq \mathcal{M}_{S_{3}}(\Gamma)$. Todos esses fatos são conseqüências do seguinte teorema, apresentado e demonstrado em [SC95]:

Teorema 3.4 (Monotonicidade) Sejam $S, S^{\prime}$ tais que $S \subseteq S^{\prime} \subseteq A$ e $\phi$ e $\psi$ sentenças em CNF. Se $\phi \forall_{S}^{1} \psi$, então $\phi \nvdash_{S^{\prime}}^{1} \psi$ (em particular, $\phi \not \models \psi$ ). Além disso, se $\phi \models_{S}^{3} \psi$, então $\phi \models_{S^{\prime}}^{3} \psi$ (em particular, $\phi \models \psi$ ).

Esse teorema é fundamental para todas as possíveis aplicações do método de Cadoli-Schaerf. Aliás, qualquer procedimento de aproximações de lógicas deve possuir uma propriedade similar. É a partir dele que se sabe que o método 
possui poder de incrementabilidade. Se, ao usar um conjunto $S$ de proposições relevantes, não se chega a nenhum resultado, aumenta-se o conjunto $S$ para um outro, $S^{\prime}$, que terá pelo menos o mesmo poder de inferência ou refutação (e possivelmente mais).

Uma observação interessante é dada pelo

Teorema 3.5 (Convergência) Sejam $\phi$ e $\psi$ sentenças em CNF. Se $\phi \models \psi$, então existe $S \subseteq A$ tal que $\phi \models_{S}^{3} \psi$. Se $\phi \not \models \psi$, então existe $S \subseteq A$ tal que $\phi \nvdash_{S}^{1} \psi$.

A demonstração desse teorema é trivial (tome $S=A$ ). Em geral, entretanto, o interesse é por conjuntos $S$ diferentes de $A$. Já que se sabe da existência de pelo menos um $S$, então sempre é possível considerar o menor $S$ que satisfaz a relação.

A idéia é a seguinte. Suponha que se deseja demonstrar que $\phi \models \psi$. Pelo Teorema 3.5, existirá $S \subseteq A$ tal que $\phi \models_{S}^{3} \psi$. Como escolher $S$ ? Considere um $S$ bastante pequeno, com apenas uma quantidade pequena de átomos. Para esse $S$, é mais fácil resolver problemas de inferência (por ser pequeno e facilmente "controlável"). Se for determinado que $\phi \models_{S}^{3} \psi$, pelo Teorema 3.4 o problema está resolvido e não há mais nada a fazer. Senão, nada pode ser afirmado. Então aumenta-se o conjunto $S$ para outro $S^{\prime} \supseteq S$ e tenta-se determinar se $\phi \models_{S^{\prime}}^{3} \psi$. Para esse conjunto, por ser um pouco maior, pode ser mais difícil a determinação de inferências, mas se sabe, pelo Teorema 3.4, que tudo o que pode ser inferido usando-se $S$ continua podendo ser inferido com $S^{\prime}$. Assim, mesmo se $S$ não resolveu o problema, o esforço computacional em resolver problemas de inferência com ele não é perdido, porque as soluções (positivas) continuam valendo para $S^{\prime}$. Considerações duais valem para a solução de problemas de refutabilidade usando aproximações por cima $S_{1}$. Cadoli e Schaerf chamam $S$ de parâmetro de qualidade de inferência.

Em [SC95] não é apresentado nenhum algoritmo que indica como incrementar os conjuntos $S$. Cadoli e Schaerf se limitam a apresentar algoritmos que reduzem $S_{1}$ ou $S_{3}$-inferência a $S_{1}$ ou $S_{3}$-satisfatibilidade e que indicam como testar $S_{1}$ ou $S_{3}$-inferência quando já se tem o conjunto $S$.

\subsection{Modalidades $\square_{S}^{1}$ e $\square_{S}^{3}$}

No trabalho de Hector Levesque, a noção de 3-aproximação é conseqüência da semântica do operador de crença implícita, $B$. Cadoli e Schaerf deram uma generalização da 3-aproximação, apresentando a $S_{3}$-aproximação, e também apresentaram sua dual, a $S_{1}$-aproximação [SC95]. Eles também formalizaram essas noções em uma lógica modal do conhecimento. Nesta, pode-se ver claramente como a $S_{3}$-aproximação soluciona o problema da onisciência lógica do agente pensante, ao passo que a $S_{1}$-aproximação está associada não ao controle da onisciência lógica, mas sim ao fato de que as " $S_{1}$-crenças" não são necessariamente verdades. 
$\mathrm{O}$ operador $B$ de Levesque se referia à sua 3-aproximação. Mas nas $S_{1}$ ou $S_{3}$-aproximações, cada conjunto $S$ está associado a uma lógica diferente. Por causa disso, torna-se necessário associar a cada $S$ dois operadores, denotados por $\square_{S}^{3}$ (para aproximações por baixo) e $\square_{S}^{1}$ (para aproximações por cima). Esses operadores podem ser aplicados a sentenças somente no formato MNNF. Sentenças MNNF são sentenças modais cujas partes proposicionais estão na forma NNF (visto que Cadoli e Schaerf lidam apenas com sentenças NNF, essa exigência é natural). Assim, sentenças são construídas a partir do conjunto $A^{\circ}$ dos literais usando os operadores binários $\wedge$ e $\vee$ e os operadores modais $\square_{S}^{1}$, $\square_{S}^{3}, \neg \square \square_{S}^{1}$ e $\neg \square_{S}^{3}$. A semântica apresentada por Cadoli e Schaerf assume que as sentenças envolvidas estão em MNNF, e generaliza o sistema $\mathbf{L}$ de Levesque.

Definição 3.6 Um modelo Cadoli-Schaerf é uma tripla $\mathcal{M}=\langle$ Sit, $R, V\rangle$, em que Sit é um conjunto não vazio de situações, $R \subseteq$ Sit $\times$ Sit é uma relação de acessibilidade e $V:$ Sit $\rightarrow\left(A^{\circ} \rightarrow\{0,1\}\right)$ é uma função que associa a cada situação uma valoração total no conjunto $A^{\circ}$ dos literais.

Compare com a definição de modelo na discussão do sistema L, Seção 1.1. A relação de acessibilidade $R$, intuitivamente, expressa quais situações podem ser "enxergadas" por um agente que se encontra em determinada situação. Defina $\mathcal{W}($ Sit $) \subseteq$ Sit como o subconjunto de situações que também são mundos possíveis, ou seja, tais que $V(s)$ também é uma função-verdade clássica ou 2interpretação. Denota-se por $S_{3}($ Sit $)$ e $S_{1}($ Sit $)$ os conjuntos de situações que são levadas a interpretações $S_{3}$ e $S_{1}$, respectivamente. A semântica é definida como segue:

CS1. $\mathcal{M}, s \models \phi$, quando $\phi$ for proposicional, sse $V(s)(\phi)=1^{2}$

CS2. $\mathcal{M}, s \models \phi \vee \psi$ sse $\mathcal{M}, s \models \phi$ ou $\mathcal{M}, s \models \psi$

CS3. $\mathcal{M}, s \models \phi \wedge \psi$ sse $\mathcal{M}, s \models \phi$ e $\mathcal{M}, s \models \psi$

CS4. $\mathcal{M}, s \models \square_{S}^{3} \phi$ sse para todo $t \in S_{3}($ Sit $)$ tal que $s R t, \mathcal{M}, t \models \phi$ $\mathcal{M}, s \models \neg \square{ }_{S}^{3} \phi$ sse existe $t \in S_{3}($ Sit $)$ tal que $s R t$ e $\mathcal{M}, t \not \models \phi$

CS5. $\mathcal{M}, s \models \square_{S}^{1} \phi$ sse para todo $t \in S_{1}$ (Sit) tal que $s R t, \mathcal{M}, t=\phi$ $\mathcal{M}, s \models \neg \square \square_{S}^{1} \phi$ sse existe $t \in S_{1}($ Sit $)$ tal que $s R t$ e $\mathcal{M}, t \not \forall \phi$

Intuitivamente, $\square_{S}^{3} \phi$ significa que a sentença $\phi$ é $S_{3}$-acreditada, ou seja, que o agente pensante aceita $\phi$ com uma leitura $S_{3}$, pela qual ele enxerga literais dentro de $S$ do ponto de vista clássico e literais fora de $S$ de um ponto de vista paraconsistente, aceitando contradições. Similarmente, $\square_{S}^{1} \phi$ significa que o agente aceita $\phi$ com uma leitura $S_{1}$, pela qual literais dentro de $S$ se comportam classicamente, mas literais fora de $S$ são sempre considerados falsos.

Uma sentença $\phi$ é válida, $\models \phi$, quando $\phi$ for verdadeira para todo mundo possível $w \in \mathcal{W}($ Sit $)$; é satisfatível quando existir um modelo $\mathcal{M}=\langle$ Sit, $R, V\rangle \mathrm{e}$

${ }^{2} \mathrm{O}$ seja, o valor verdade de $\phi$, calculado utilizando as Regras $\vee$ e $\wedge$ dadas na Seção 3.1, é igual a 1. 
um mundo possível $w \in \mathcal{W}($ Sit $)$ tal que $\mathcal{M}, w \models \phi$. Uma questão fundamental é a relação entre os operadores $\square_{S}^{1}$ e $\square_{S}^{3}$ e suas respectivas relações de inferência $\models_{S}^{1} \mathrm{e} \models_{S}^{3}$. Ela é dada pelos resultados abaixo, apresentados e demonstrados em [SC95] (aqui, $\models \square_{S}^{3} \phi \supset \square_{S}^{3} \psi$ é uma abreviação para $\models \neg \square_{S}^{3} \phi \vee \square_{S}^{3} \psi$ ):

Teorema 3.7 (Validade modal e $S_{3}$-inferência) Sejam $\phi$ e $\psi$ duas sentenças proposicionais. Então $\models \square_{S}^{3} \phi \supset \square_{S}^{3} \psi$ sse $\square_{S}^{3} \phi \wedge \neg \square_{S}^{3} \psi$ for insatisfativel classicamente sse $\phi \models_{S}^{3} \psi$.

Teorema 3.8 (Validade modal e $S_{1}$-inferência) Sejam $\phi$ e $\psi$ duas sentenças proposicionais. Então $\models \square_{S}^{1} \phi \supset \square_{S}^{1} \psi$ sse $\square_{S}^{1} \phi \wedge \neg \square_{S}^{1} \psi$ for insatisfativel classicamente sse $\phi \models_{S}^{1} \psi$.

Esses teoremas ligam o conceito de aproximações de lógicas aos operadores $\square_{S}^{1}$ e $\square_{S}^{3}$. Um outro resultado, também de [SC95] e extremamente importante, faz a ligação entre os diversos operadores $\square_{S}^{3}$ para vários conjuntos de proposições relevantes $S$ :

Teorema 3.9 (Monotonicidade para $\square_{S}^{3}$ ) Seja $S \subseteq S^{\prime} \subseteq A$. Então, tem-se $q u e \models \square_{S}^{3} \phi \supset \square_{S^{\prime}}^{3} \psi$.

Cadoli e Schaerf observam que, como o sistema modal S5 é geralmente aceito como uma formalização apropriada da noção de conhecimento, deve-se assumir que a relação de acessibilidade $R$ é reflexiva, transitiva e euclidiana. É importante comparar quais dos axiomas e regras de inferência que caracterizam S5 continuam valendo quando se substitui o operador modal $\square$ por $\square_{S}^{1}$ e $\square_{S}^{3}$. O sistema S5, apresentado na Seção $2.2 .1^{3}$, possui uma única regra de inferência modal,

Regra da Necessitação: se $\phi$ é um teorema, então $\square \phi$ também é um teorema

e os seguintes axiomas:

$$
\begin{aligned}
& \text { K. } \square(p \supset q) \supset(\square p \supset \square q) \\
& \text { T. } \square p \supset p \\
& \text { 4. } \square p \supset \square \square p \\
& \text { 5. } \neg \square p \supset \square \neg \square p
\end{aligned}
$$

Cadoli e Schaerf observam que a Regra da Necessitação não vale quando se troca $\square$ por $\square{ }_{S}^{1}$. O contra-exemplo proposto é o seguinte. Seja $\mathcal{M}=\langle$ Sit, $R, V\rangle$, de forma que:

$$
\text { - } \phi=q \vee \neg q
$$

${ }^{3} \mathrm{O}$ conjunto de axiomas apresentado aqui é um pouco diferente daquele apresentado na Seção 2.2.1: lá, S5 possui os axiomas $K, T$ e 5 ; aqui, o axioma 5 é substituído por 4 e uma outra versão de 5 . Os dois sistemas são equivalentes, mas o último não possui, em sua formulação, nenhum axioma com o operador 0 . Como Cadoli e Schaerf trabalharam unicamente com o operador $\square$ (e suas variantes $\square_{S}^{1}$ e $\square_{S}^{3}$ ), essa formulação é preferida aqui. 
- $S=\emptyset$, Sit $=\left\{s_{1}, s_{2}\right\}, R=$ Sit $\times$ Sit

- $V\left(s_{1}\right)(q)=1$

$V\left(s_{1}\right)(\neg q)=V\left(s_{2}\right)(q)=V\left(s_{2}\right)(\neg q)=0$

A função $V$ é, para cada situação, uma $S_{1}$-interpretação. Logo, $S_{1}($ Sit $)=$ $\left\{s_{1}, s_{2}\right\}$. Além disso, $\mathcal{W}($ Sit $)=\left\{s_{1}\right\}$. Como $s_{1}$ é o único mundo possível em relação ao conjunto Sit, e para $s_{1}$ a sentença $q \vee \neg q$ é verdadeira, então $\mathcal{M} \models \phi$. Entretanto, $\mathcal{M} \not \models \square_{S}^{1} \phi$, uma vez que $\mathcal{M}, s_{2} \not \models q \vee \neg q$ e, $s_{1}$ acessando $s_{2}, \mathcal{M}, s_{1} \not \models \square_{S}^{1}(q \vee \neg q)$.

A validade de $K$ segue da definição semântica de $\square_{S}^{1}$. Por causa da transitividade de $R$, que é assumida, vale 4 . O princípio 5 vale, como pode ser visto no argumento que segue. Suponha que $\mathcal{M}, w \models \neg \square_{S}^{1} \phi$ para algum modelo CadoliSchaerf $\mathcal{M}$ e um mundo possível $w$ desse modelo. Então, por CS5, tem-se que existe $t \in S_{1}($ Sit) tal que $w R t$ e $\mathcal{M}, t \not \models \phi$. Pelo fato de $R$ ser euclidiana e reflexiva, segue que ela é simétrica. Assim, dada qualquer situação $v-$ em particular para as $v \in S_{1}($ Sit $)$ - tal que $w R v$, tem-se que $v R w$; a transitividade implica que $v R t$ e, portanto, que $\mathcal{M}, v \models \neg \square{ }_{S}^{1} \phi$. Como isso é verdade para toda $v \in S_{1}($ Sit $)$ tal que $w R v$, então $\mathcal{M}, w \models \square_{S}^{1} \neg \square_{S}^{1} \phi$.

O princípio $T$ não vale, e o contra-exemplo de [SC95] é o seguinte. Seja $\mathcal{M}=\langle$ Sit $, R, V\rangle$, de forma que:

- $S=\emptyset$, Sit $=\left\{s_{1}\right\}, R=\left\{\left(s_{1}, s_{1}\right)\right\}$

- $V\left(s_{1}\right)(\neg p)=1$

$V\left(s_{1}\right)(p)=0$

A função $V$, aplicada em $s_{1}$, não dá uma $S_{1}$-interpretação. Logo $S_{1}($ Sit $)=\emptyset$. Além disso, $\mathcal{W}($ Sit $)=\left\{s_{1}\right\}$. Por vacuidade, $\mathcal{M}, s_{1} \models \square_{S}^{1} p$, mas $\mathcal{M}, s_{1} \not \neq p$. Portanto, tem-se que $\mathcal{M}, s_{1} \not \models \square_{S}^{1} p \supset p$.

Cadoli e Schaerf observam [SC95] que tanto a regra da necessitação como o esquema de axiomas $T$ não valem em geral. Como conseqüência, é possível usar $\square_{S}^{1}$ para modelar um agente capaz de realizar pelo menos todas as inferências corretas, porque seu conhecimento é fechado sob modus ponens (o esquema $K$ ); entretanto, o agente pode fazer algumas inferências que não são corretas, pois de fato o esquema $T$ não vale. Uma vez que os esquemas 4 e 5 são válidos, segue que os agentes modelados no sistema são totalmente introspectivos. A questão da introspeção total ou ilimitada é abordada com mais detalhes no Capítulo 4 .

O que ocorre quando se substitui $\square$ por $\square_{S}^{3}$ ?

Vale a regra da necessitação e pela seguinte razão. Se $\models \phi$, então para todo modelo $\mathcal{M}=\langle$ Sit, $R, V\rangle$ e todo mundo possível $w$ desse modelo, ocorre $\mathcal{M}, w \models \phi$. Se não ocorresse $\mathcal{M}, w \models \square_{S}^{3} \phi$, então existiria um $t \in S_{3}($ Sit $)$ tal que $w R t$ e $\mathcal{M}, t \not \models \phi$. Isso por si só, até que poderia ocorrer a princípio, pois $t$ é uma situação e não um mundo possível. Mas suponha que $\phi$ seja proposicional - nesse caso, $\phi$ está em NNF. Sabe-se, por hipótese, que toda 2-interpretação deve satisfazer $\phi$. Uma $S_{3}$-interpretação irá diferir da 2-interpretação porque que certos pares de literais, que na 2-interpretação recebem valores opostos, são ambos mapeados em 1. Para uma sentença $\phi$ em NNF, isso só aumentará 
as chances de $\phi$ ser verdadeira. Assim, se toda 2-interpretação satisfizer $\phi$, toda $S_{3}$-interpretação também a satisfará. Portanto, para todo $t \in S_{3}$ (Sit), $\mathcal{M}, t \models \phi$ (em particular, para aqueles tais que $w R t)$. É importante observar que a validade desse argumento depende do fato de $\phi$ estar em NNF. Suponha, a seguir, que $\phi$ seja da forma $\psi_{1} \vee \psi_{2}$, e que já tenha sido provado que, se $\mathcal{M}, w \models$ $\psi_{1}$ e $\mathcal{M}, w \models \psi_{2}$ para todo mundo possível $w$ de um modelo $\mathcal{M}=\langle$ Sit, $R, V\rangle$ genérico, então $\mathcal{M}, t \models \psi_{1}$ e $\mathcal{M}, t \models \psi_{2}$ para toda situação $t \in S_{3}($ Sit). Então $\mathcal{M}, w \models \psi_{1} \vee \psi_{2}$ sse $\mathcal{M}, w \models \psi_{1}$ ou $\mathcal{M}, w \models \psi_{2}$, que implica que $\mathcal{M}, t \models \psi_{1}$ ou $\mathcal{M}, t \models \psi_{2}$, que ocorre sse $\mathcal{M}, t \models \psi_{1} \vee \psi_{2}$. Similarmente, mostra-se o resultado se $\phi=\psi_{1} \wedge \psi_{2}$. Suponha, agora, que $\phi$ seja da forma $\square_{S}^{3} \psi$, e que já tenha sido mostrado que, se $\mathcal{M}, w \models \psi$ para todo mundo possível $w$ de um modelo $\mathcal{M}=\langle$ Sit, $R, V\rangle$ genérico, então $\mathcal{M}, t \models \psi$ para toda situação $t \in S_{3}($ Sit $)$. Ocorre $\mathcal{M}, t \models \square_{S}^{3} \psi$ sse, para todo $v \in S_{3}(S i t)$ tal que $t R v$, ocorrer $\mathcal{M}, v \models \psi$. Mas isso já acontece por hipótese, logo $\mathcal{M}, t \models \square_{S}^{3} \psi$ para todo $t \in S_{3}$ (Sit). Por fim, suponha que $\phi$ seja da forma $\neg \square_{S}^{3} \psi$. Então $\mathcal{M}, w \models \neg \square{ }_{S}^{3} \psi$ sse existe $t \in S_{3}$ (Sit) tal que $w R t$ e $\mathcal{M}, t \not \models \psi$. Considere $v \in S_{3}($ Sit) tal que $w R v$. Pela propriedade euclidiana da relação $R, v R t$. Portanto, $\mathcal{M}, v \models \neg \square_{S}^{3} \psi$. Isso vale para todo $v \in S_{3}(S i t)$ tal que $w R v$. O que foi mostrado, portanto, é que se $\mathcal{M}, w \models \phi$, então $\mathcal{M}, t \models \phi$ para todo $t \in S_{3}($ Sit) tal que $w R t$, e isso implica que $\mathcal{M}, w \models \square_{S}^{3} \phi$, o que mostra a validade da regra da necessitação.

O princípio $T$ vale porque toda 2-interpretação é uma $S_{3}$-interpretação, além do fato de que a relação de acessibilidade é reflexiva. Os princípios 4 e 5 , cuja demonstração para $\square_{S}^{1}$ dependiam exclusivamente das propriedades da relação de acessibilidade $R$, continuam valendo igualmente para $\square_{S}^{3}$.

Para a propriedade $K$, Cadoli e Schaerf oferecem um contra-exemplo, também de [SC95], que é o seguinte. Seja $\mathcal{M}=\langle$ Sit, $R, V\rangle$, de forma que:

- $S=\emptyset$, Sit $=\left\{s_{1}, s_{2}\right\}, R=$ Sit $\times$ Sit

- $V\left(s_{1}\right)(p)=V\left(s_{1}\right)(q)=V\left(s_{2}\right)(p)=V\left(s_{2}\right)(\neg p)=V\left(s_{2}\right)(\neg q)=1$ $V\left(s_{1}\right)(\neg p)=V\left(s_{1}\right)(\neg q)=V\left(s_{2}\right)(q)=0$

A função $V$ é, para cada elemento de $S i t$, uma $S_{3}$-interpretação. Dessa forma, $S_{3}($ Sit $)=$ Sit. Além disso, $\mathcal{W}($ Sit $)=\left\{s_{1}\right\}$. Tem-se que $\mathcal{M}, s_{1} \vDash p$ e $\mathcal{M}, s_{1} \models q$; assim, que $\mathcal{M}, s_{1} \vDash p \supset q$. Da mesma forma (e lembrando que, para CadoliSchaerf, $p \supset q$ é equivalente a $\neg p \vee p$, já que eles trabalham com fórmulas em NNF), como ocorre $\mathcal{M}, s_{2} \models \neg p$, tem-se $\mathcal{M}, s_{2} \vDash p \supset q$. Daí segue que $\mathcal{M}, s_{1} \models \square_{S}^{3}(p \supset q)$. Como $\mathcal{M}, s_{2} \models p$, tem-se também que $\mathcal{M}, s_{1} \models \square_{S}^{3} p$. Porém, como $V\left(s_{2}\right)=0$, tem-se que $\mathcal{M}, s_{2} \not \models q$. A situação $s_{1}$ é relacionada com $s_{2}$, e isso implica, pela condição CS5, que $\mathcal{M}, s_{1} \not \models \square_{S}^{3} q$.

Em suma, o princípio $K$, que engloba o conceito de onisciência lógica, não vale para o operador $\square_{S}^{3}$. Com isso, a utilização das $S_{3}$-aproximações soluciona o problema da onisciência lógica: apenas a partir de fatos relacionados mais de perto com aquilo que é de interesse (ou seja, com o conjunto das proposições relevantes $S$ ) podem-se fazer inferências. De fatos que utilizam apenas proposições fora do conjunto de proposições relevantes nada se pode inferir; e inconsistências que eventualmente ocorram nesses fatos não afetam a correção das inferências 
feitas dentro de $S$. A validade do princípio $T$ implica que só são feitas inferências corretas, como já havia sido mostrado no Teorema 3.4. Por essas razões, nas próximas seções serão enfatizadas principalmente as aproximações por baixo, todas elas tendo sido inspiradas pelas $S_{3}$-aproximações de Cadoli e Schaerf.

Suponha que $S=\emptyset$. Denomine por $\mathcal{B}$ o conjunto $S_{3}($ Sit $)$. A relação $R$, sendo uma relação de equivalência, particiona o conjunto Sit em classes de equivalência. Com isso, a condição $s R t$ pode ser retirada pois, dadas duas situações $s$ e $t$, ou elas se relacionam e são equivalentes, ou não se relacionam - logo, pode-se considerar Sit como uma união de classes universais disjuntas. As condições de verdade de $\square_{\emptyset}^{3}$ são, portanto:

$$
\begin{aligned}
& \mathcal{M}, s \models \square_{\emptyset}^{3} \phi \text { sse para todo } t \in \mathcal{B}, \mathcal{M}, t \models \phi \\
& \mathcal{M}, s \models \neg \square_{\emptyset}^{3} \phi \text { sse existe } t \in \mathcal{B} \text { tal que } \mathcal{M}, t \not \models \phi
\end{aligned}
$$

Essa é justamente a definição de verdade L5, dada no Capítulo 1 . Portanto, o operador $\square_{\emptyset}^{3}$ é semanticamente igual ao operador de crença implícita $B$ de Levesque.

Se $S=A$, os conjuntos $S_{3}\left(\right.$ Sit) e $S_{1}$ (Sit) são idênticos, dados pelo conjunto das situações levadas a 2-interpretações (e portanto que são mundos possíveis). Dessa forma, $S_{3}($ Sit $)=S_{1}($ Sit $)=\mathcal{W}($ Sit $)$. As condições de verdade de $\square_{A}^{3}$ (que são iguais às de $\square_{A}^{1}$ ) são:

$$
\begin{aligned}
& \mathcal{M}, s \models \square \square_{A}^{3} \phi \text { sse para todo } t \in \mathcal{W}(\text { Sit }), \mathcal{M}, t \models \phi \\
& \mathcal{M}, s \models \neg \square_{A}^{3} \phi \text { sse existe } t \in \mathcal{W}(\text { Sit }) \text { tal que } \mathcal{M}, t \not \models \phi
\end{aligned}
$$

Essa é a definição de verdade L6. Portanto, os operadores $\square_{A}^{3}$ e $\square_{A}^{1}$ são semanticamente iguais ao operador de crença implícita $L$ de Levesque. ${ }^{4}$

Uma observação final: vale o seguinte

Teorema $3.10 \models \square_{A}^{3} \phi \supset \phi$.

Dem: Suponha que, para um modelo $\mathcal{M}$ e uma situação $s$, ocorra $\mathcal{M}, s \models$ $\square_{A}^{3} \phi$. Então para todo $t \in \mathcal{W}($ Sit $), \mathcal{M}, t \models \phi$. Isso vale em particular para a situação $s$, pois quando o conjunto de proposições relevantes, $S$, é igual a $A$, todas as situações são mundos possíveis. Portanto, $\mathcal{M}, s \models \phi$.

Com esse resultado, o Teorema 3.9 implica (para $S^{\prime}=A$ ) que $\models \square_{S}^{3} \phi \supset \phi$. Ou seja, se uma sentença é $S_{3}$-acreditada, ela é verdadeira. Uma demonstração similar mostra que $\models \square_{A}^{1} \phi \supset \phi$.

\subsection{Aplicação a Lógicas Modais}

Na Seção 3.2, mostrou-se como o método de aproximação pode ser formalizado em uma linguagem modal da teoria do conhecimento; as relações $\models_{S}^{1} \mathbf{e} \models_{S}^{3}$, nesse

\footnotetext{
${ }^{4}$ Embora as semânticas de $B$ e $L$ possam ser recuperadas pelo método de Cadoli-Schaerf, existe uma diferença. No sistema $L$ de Levesque, seleciona-se um subconjunto $\mathcal{B} \subseteq \mathcal{S}$ de situaçōes acreditadas. Sobre esse conjunto, a semântica de $B$ é a mesma que a do operador $\square_{A}^{3}$ sobre o conjunto $S_{3}($ Sit $)$. A semântica de $L$ é feita no conjunto $\mathcal{W}(\mathcal{B})$, que é a mesma que a do operador $\square_{A}^{3}$ sobre o conjunto $\mathcal{W}($ Sit $)$. Vale $\square_{\emptyset}^{3} \phi \supset \square_{A}^{3} \phi$ assim como vale $B \phi \supset L \phi$, mas não existe um equivalente ao conjunto $\mathcal{B}$ no método de Cadoli-Schaerf.
} 
caso, são associadas a dois operadores modais $\square_{S}^{1}$ e $\square_{S}^{3}$. Entretanto, isso não representou uma extensão do método de Cadoli-Schaerf para uma linguagem modal que, em adição aos operadores clássicos $\neg, \wedge, \vee, \equiv$, possui os dois operadores modais necessitação, $\square$, e possibilitação, $\diamond$. Nesta seção, serão mostrados alguns dos resultados de [SC95] nessa direção.

Cadoli e Schaerf se restringem a sentenças modais na forma MNNF (ver Seção 3.2). Lembrando da definição dada na Seção 2.2.1, um modelo de Kripke é uma tripla $\mathcal{M}=\langle W, R, V\rangle$, em que $W$ é um conjunto não vazio de mundos possíveis, $R \subseteq W \times W$ é uma relação de acessibilidade e $V: W \rightarrow\left(A^{\circ} \rightarrow\right.$ $\{0,1\})$ é uma função que associa a cada mundo possível uma 2-interpretação do conjunto dos literais 5 .

Em um modelo de Kripke padrão, $V(w)$ é uma 2-interpretação para cada mundo $w \in W$. Quando isso ocorre, será utilizada a expressão interpretação 2-Kripke. Cadoli e Schaerf dão outras duas definições:

Definição 3.11 (Interpretação $S_{1}$-Kripke) Uma interpretação $S_{1}$-Kripke é um modelo de Kripke tal que $V(w)$ é uma $S_{1}$-interpretação para todo mundo $w \in W$.

Definição 3.12 (Interpretação $S_{3}$-Kripke) Uma interpretação $S_{3}$-Kripke é um modelo de Kripke tal que $V(w)$ é uma $S_{3}$-interpretação para todo mundo $w \in W$.

Quando uma interpretação $S_{1}$-Kripke $\mathcal{M}$ satisfaz uma sentença em um mundo possível $w \in W$, utiliza-se a notação $\mathcal{M}, w \models_{S}^{1} \phi$. Similarmente, para interpretações $S_{3}$-Kripke, utiliza-se a notação $\mathcal{M}, w \models_{S}^{3} \phi$. A semântica dessas relações é dada por

CSM1. $\mathcal{M}, w \models_{S}^{3} \phi$, quando $\phi$ é proposicional, sse $V(w)(\phi)=1$

$\mathcal{M}, w \models{ }_{S}^{1} \phi$, quando $\phi$ é proposicional, sse $V(w)(\phi)=1$

CSM2. $\mathcal{M}, w \models_{S}^{3} \phi \wedge \psi$ sse $\mathcal{M}, w \models_{S}^{3} \phi$ e $\mathcal{M}, w \models_{S}^{3} \psi$

$\mathcal{M}, w \models{ }_{S}^{1} \phi \wedge \psi$ sse $\mathcal{M}, w \models{ }_{S}^{1} \phi$ e $\mathcal{M}, w \models_{S}^{1} \psi$

CSM3. $\mathcal{M}, w \models_{S}^{3} \phi \vee \psi$ sse $\mathcal{M}, w \models_{S}^{3} \phi$ ou $\mathcal{M}, w \models_{S}^{3} \psi$

$\mathcal{M}, w \models_{S}^{1} \phi \vee \psi$ sse $\mathcal{M}, w \models_{S}^{1} \phi$ ou $\mathcal{M}, w \models_{S}^{1} \psi$

CSM4. $\mathcal{M}, w \models_{S}^{3} \square \phi$ sse para todo $t \in W$ tal que $w R t, \mathcal{M}, t \models_{S}^{3} \phi$ $\mathcal{M}, w \models_{S}^{3} \neg \square \phi$ sse existe $t \in W$ tal que $w R t$ e $\mathcal{M}, t \models_{S}^{3} \neg \phi$

CSM5. $\mathcal{M}, w \models_{S}^{1} \square \phi$ sse para todo $t \in W$ tal que $w R t, \mathcal{M}, t \models_{S}^{1} \phi$

$\mathcal{M}, w \models{ }_{S}^{1} \neg \square \phi$ sse existe $t \in W$ tal que $w R t$ e $\mathcal{M}, t \models_{S}^{1} \neg \phi$

\footnotetext{
${ }^{5} \mathrm{Na}$ Seção 2.2.1, a formulação é um pouco diferente, porque não se definia a valoração $V$ como uma função de $W$ em $A^{\circ} \rightarrow\{0,1\}$, e sim como uma função de $W \times A$ em $\{0,1\}$. A razão da diferença está que, na Seção 2.2.1, a verdade era determinada unicamente a partir dos átomos; aqui, trabalham-se com conceitos em que uma valoração somente dos átomos não é suficiente. Mas, para modelos de Kripke clássicos, as definições são equivalentes.
} 
Cadoli e Schaerf não dão uma semântica para o operador $\diamond$; provavelmente eles entendem $\diamond \phi$ como outra forma de escrever $\neg \square \neg \phi$, em que $\neg \phi$ seria o equivalente clássico da negação de $\phi$ em NNF.

Definiçōes dos conceitos de $S_{1}$-Kripke-satisfatibilidade, $S_{3}$-Kripke-satisfatibilidade, $S_{1}$-Kripke-insatisfatibilidade, $S_{3}$-Kripke-insatisfatibilidade, $S_{1}$-Kripkevalidade, $S_{1}$-Kripke-validade e $S_{3}$-Kripke-validade são dadas da maneira padrão. Satisfatibilidade, insatisfatibilidade e validade para 2-Kripke interpretações são referidas como 2-Kripke-satisfatibilidade, etc. A condição de CSM1 deve ser interpretada como segue: $V(w)(\phi)$ é calculado como o valor verdade dado na Seção 3.1.

Para CSM4 e CSM5, há algumas observações importantes. Se essas condiçōes forem comparadas com suas equivalentes CS4 e CS5, da Seção 3.2, notam-se diferenças e analogias.

As condições CS4 e CS5 são definidas para situações, que sāo uma generalização do conceito de mundos possíveis. Isso é necessário para a expressão da semântica dos operadores $\square_{S}^{1}$ e $\square_{S}^{3}$; esses operadores representam, intuitivamente, as crenças de um agente pensante limitado. Esse agente pode acreditar em certas coisas que, em uma análise mais acurada, mostram-se contraditórias. Foi para não trivializar as conclusões do agente nesses casos (ou seja, dizer que ele acredita em tudo se ele acredita em uma contradição) que se construiu o conceito de situações. Já em CSM4 e CSM5, o interesse é estabelecer um método que aproxime a Lógica Modal Clássica. Problemas de satisfatibilidade de lógicas modais são reconhecidamente complexos computacionalmente (como estudados em [Lad77]). Por exemplo, mostra-se que o problema de satisfatibilidade do sistema mais simples de lógica modal, $S 5$, é NP-completo; outros sistemas conhecidos têm problemas PSPACE-completos. Uma primeira abordagem, como essa de Cadoli-Schaerf, procura controlar o poder de inferência em cada mundo, mas não mexer no próprio conceito de mundo possível. É como se cada mundo possível representasse um agente pensante sob o qual se aplicassem as condições de verdade da Seção 3.1 .

Uma segunda observação é que $\square_{S}^{1}$ e $\square_{S}^{3}$ são familias de operadores, um par para cada $S \subseteq A$. Uma interação desses diversos operadores faz parte do estudo das lógicas multimodais. Já a lógica que é estudada nesta seção é monomodal, com a existência de apenas um operador $\square$. Para uma eventual extensão do estudo para lógicas multimodais, são necessárias a determinação e o estudo das propriedades de uma lógica monomodal. Na Seção 3.2, o estudo da interação dos operadores era um importante aspecto para que se compreendessem os mesmos e não poderia ser dispensado para se ter maior simplicidade.

A terceira, e mais importante, observação refere-se à semântica dada às formas $\neg \square \square_{S}^{1} \phi \mathbf{e} \neg \square{ }_{S}^{3} \phi$, por um lado, e $\neg \square \phi$, por outro. Nas sentenças do primeiro caso, é impossível, pelas definiçōes CS4 e CS5, que ocorra simultaneamente $\square_{S}^{3} \phi \wedge \neg \square \square_{S}^{3} \phi$. Isso porque a relação de validade, $\models$, funciona "classicamente", por assim dizer - e, portanto, afirmar $\square_{S}^{3} \phi \wedge \neg \square_{S}^{3} \phi$ significa afirmar, ao mesmo tempo, que existe uma situação $t$ tal que $\mathcal{M}, t \models \square_{S}^{3} \phi$ e $\mathcal{M}, t \not \models \square_{S}^{3} \phi$, contradição. Por outro lado, se $\phi \wedge \neg \phi$ vale em uma interpretação $S_{3}$ (e o Exemplo 4 da Seção 1.1 mostra que vale), então segue da definição CSM4 que $\mathcal{M}, w \models_{S}^{3} \square \phi$ 
e $\mathcal{M}, w \models_{S}^{3} \neg \square \phi$ para algum modelo $\mathcal{M}$ (basta tomar um modelo com um único mundo, com uma relaçāo de acessibilidade que é uma relação de equivalência (reflexiva, simétrica e transitiva) e tendo por $V(w)$ a interpretaçāo $S_{3}$ que satisfaz $\phi \mathrm{e} \neg \phi)$. Então, $\mathcal{M}, w \models_{S}^{3} \square \phi \wedge \neg \square \phi$.

Cadoli e Schaerf, em [SC95], explicam suas escolhas para as semânticas dos dois tipos de operadores. Segundo eles, as condições CS4 e CS5 constróem uma linguagem capaz de representar as relações de inferência aproximada, mas não são boas do ponto de vista técnico. Por outro lado, CSM4 e CSM5 são condições que fazem a linguagem computacionalmente interessante; [SC95] apresenta alguns resultados de complexidade uniforme para essa linguagem.

Cadoli e Schaerf apresentam e demonstram, em [SC95], alguns teoremas obtidos a partir das definições acima:

Teorema 3.13 (Monotonicidade) Para todos $S, S^{\prime}$ tais que $S \subseteq S^{\prime} \subseteq A$, se $\phi$ for $S_{1}$-Kripke-satisfativel, então $\phi$ é $S_{1}^{\prime}$-Kripke-satisfativel (e portanto 2Kripke-satisfativel). Além disso, se $\phi$ for $S_{3}$-Kripke-insatisfativel, entäo $\phi \dot{e}$ $S_{3}^{\prime}$-Kripke-insatisfativel (e portanto 2-Kripke-insatisfativel).

Teorema 3.14 (Convergência) Se $\phi$ for 2-Kripke-satisfativel, então existe um subconjunto $S \subseteq A$ tal que $\phi$ é $S_{1}$-Kripke-satisfativel. Se $\phi$ for 2-Kripkeinsatisfativel, então existe um $S \subseteq A$ tal que $\phi$ é $S_{3}$-Kripke-insatisfativel.

O Teorema 3.14 é de demonstração trivial: basta tomar $S=A$, pois nesse caso $S_{1}$-Kripke-satisfatibilidade é igual a 2-Kripke-satisfatibilidade, e similarmente para $S_{3}$-Kripke-insatisfatibilidade. Esses teoremas permitem a obtenção de resultados de 2-Kripke-satisfatibilidade e 2-Kripke-insatisfatibilidade a partir da obtenção de $S_{1}$-Kripke-satisfatibilidade e $S_{3}$-Kripke-insatisfatibilidade, para algum conjunto $S$. Naturalmente, os últimos resultados são mais fáceis de serem obtidos, como mostram dois exemplos de [SC95] e reproduzidos abaixo.

1. Seja $L=\{p, q, r\}, S=\{p, q\}$ e $\phi=\square p \wedge \square(\neg p \vee q) \wedge \square \neg q \wedge \neg \square r$. Suponha que $\phi$ é $S_{3}$-Kripke-satisfatível. Então existe um modelo $\mathcal{M}$ e um $w \in W$ tal que, para todo $t \in W$ que satisfaz $w R t$, devem ser satisfeitas as seguintes condições: $\mathcal{M}, t \models_{S}^{3} p, \mathcal{M}, t \models_{S}^{3} \neg p \vee q$ e $\mathcal{M}, t \models_{S}^{3} \neg q$. Além disso, existe um $v \in W$ tal que $w R v$ e que deve satisfazer $\mathcal{M}, v \models_{S}^{3} \neg r$. Esse $v$, cuja existência é necessária se for assumido que $\phi$ é $S_{3}$-Kripke-satisfatível, deveria considerar verdadeiras as sentenças $p, \neg p \vee q \mathrm{e} \neg q$. Só que $p \mathrm{e}$ $q$, pertencendo ao conjunto das proposiçōes relevantes $S$, devem se comportar classicamente e, portanto, é impossível que sejam simultaneamente verdadeiras as sentenças $p, \neg p \vee q$ e $\neg q$. Observe que, se $S^{\prime}=\{p\}$ apenas, $\phi$ é $S^{\prime}-3$-Kripke-satisfatível, pois nesse caso $q$ e $\neg q$ podem ser ambas verdadeiras em $v$.

2. Seja $L=\{p, q\}, S=\{q\}$ e $\phi=\neg q \wedge \square p \wedge \square(\neg p \vee q)$. Considere o seguinte modelo: $W=\{w\}, R=\emptyset, V(w)(\neg q)=1$ e $V(w)(p)=V(w)(\neg p)=$ $V(w)(q)=0$. Assim, $V(w)$ é uma $S_{1}$-interpretação. Para esse modelo $\mathcal{M}$ e no mundo $w$, ocorre $\mathcal{M}, w \models_{S}^{1} \neg q$. Por vacuidade, também é verdade 
que, para todo $t \in W$ tal que $w R t, \mathcal{M}, t \models_{S}^{1} p$ e $\mathcal{M}, t \models_{S}^{1} \neg p \vee q$, o que implica que $\mathcal{M}, w \models_{S}^{1} \square p$ e $\mathcal{M}, t \models_{S}^{1} \square(\neg p \vee q)$. Portanto, $\phi$ é $S_{1}$-Kripkesatisfatível.

Cadoli e Schaerf também apresentaram outra semântica para os operadores modais que permite um maior controle sobre a complexidade dos problemas. Isso é feito limitando-se o poder de introspecção de uma sentença, ou seja, o número de interações entre os operadores modais. Para cada número inteiro $i$, a semântica consegue determinar a verdade de um mundo $w$ em sentenças que exijam, para sua determinação de verdade, apenas a determinação de verdade de suas subsentenças em mundos cuja "distância" a $w$ seja menor ou igual a $i$.

Seja $\phi$ uma sentença modal, $n$ sua profundidade modal (ou seja, cada ocorrência de um operador modal ocorre no escopo de no máximo $n$ operadores modais), $S \subseteq A, i \leq n+1$ e $\mathcal{M}=\langle W, R, V\rangle$ uma interpretação $S_{3}$-Kripke. Define-se a relação $\models_{S, i}^{3}$ como segue:

CSD1. $\mathcal{M}, w \models_{S, i}^{3} \phi$, quando $\phi$ é proposicional, sse $V(w)(\phi)=1$ ou $i<0$

CSD2. $\mathcal{M}, w \models_{S, i}^{3} \phi \wedge \psi$ sse $\mathcal{M}, w \models_{S, i}^{3} \phi$ e $\mathcal{M}, w \models_{S, i}^{3} \psi$

CSD3. $\mathcal{M}, w \models_{S, i}^{3} \phi \vee \psi$ sse $\mathcal{M}, w \models_{S, i}^{3} \phi$ ou $\mathcal{M}, w \models_{S, i}^{3} \psi$

CSD4. $\mathcal{M}, w \models_{S, i}^{3} \square \phi$ sse para todo $t \in W$ tal que $w R t, \mathcal{M}, w \models_{S, i-1}^{3} \phi$ $\mathcal{M}, w \models_{S, i}^{3} \neg \square \phi$ sse existe $t \in W$ tal que $w R t$ e $\mathcal{M}, t \models_{S, i-1}^{3} \neg \phi$

Nota-se que, quando $i<0$, a sentença $\phi$ é sempre verdadeira em $w$, independente do que seja. Assim, sentenças com profundidade modal maior que um certo $j$ são sempre consideradas verdadeiras para $w$ sob a relação $\models_{S, j}^{3}$. Isso corresponde, intuitivamente, ao fato de que, para mundos muito "distantes", tudo é aceito (já que não se pode verificar se isso ocorre a não ser que se tenha mais recursos, o que corresponde a aumentar $j$ ). Assim, as interpretações $S_{3}$-Kripke sob essa semântica são, utilizando a expressão de Cadoli e Schaerf, "otimistas" para os mundos fora do alcance de $w$.

A definição para o caso $S_{1}$ é similar, com a diferença que a cláusula CSD1 é substituída por

$$
\mathcal{M}, w \models_{S, i}^{1} \phi \text {, quando } \phi \text { é proposicional, sse } V(w)(\phi)=1 \text { e } i \geq 0
$$

Dessa forma, quando $i<0$, a sentença $\phi$ é sempre falsa em $w$. Cadoli e Schaerf utilizam a expressão "pessimistas" para se referirem às interpretações $S_{1}$-Kripke para os mundos fora do alcance de $w$.

Essas definições são naturais quando se lembra que, no caso puramente proposicional, dado na Seção 3.1, interpretações $S_{1}$ consideram falsos todos os literais fora do conjunto de proposições relevantes $S$.

Vale um análogo ao Teorema 3.13, que não é demonstrado em [SC95] mas que o será a seguir: 
Teorema 3.15 (Monotonicidade) Seja $S \subseteq S^{\prime} \subseteq A$ e $i \leq j \leq n+1$. Se uma sentença modal $\phi$ é $\left\langle S_{3}, i\right\rangle$-Kripke-insatisfatível, então ela é $\left\langle S_{3}^{\prime}, j\right\rangle$-Kripkeinsatisfativel. Se ela for $\left\langle S_{1}, i\right\rangle$-Kripke-satisfatível, então ela é $\left\langle S_{1}^{\prime}, j\right\rangle$-Kripkesatisfativel.

Dem: Esse teorema é um corolário do Teorema 3.13. Se $i=j$, a demonstração é a mesma, e pode ser encontrada em [SC95]. Assim, se $\phi$ é $\left\langle S_{3}, i\right\rangle$ Kripke-insatisfatível, ela é $\left\langle S_{3}^{\prime}, i\right\rangle$-Kripke-insatisfatível, e se $\phi$ for $\left\langle S_{1}, i\right\rangle$-Kripkesatisfatível, ela também é $\left\langle S_{1}^{\prime}, i\right\rangle-1$-Kripke-satisfatível. Suponha, dessa forma, que $i<j$ e que $S=S^{\prime}$. As condições CSD2 e CSD3 independem do índice $i$ e, portanto, se valem (ou não valem) para $i$, continuam valendo (ou não valendo) para $j$. A condição CSD4 faz com que a determinação da $\left\langle S_{1}, i\right\rangle$ ou $\left\langle S_{3}, i\right\rangle$-verdade de uma sentença da forma $\square \phi$ seja dependente da $\left\langle S_{1}, i-1\right\rangle$ ou $\left\langle S_{3}, i-1\right\rangle$-verdade de uma sentença da forma $\phi$. Então essa condição acaba sendo levada, em última análise, à condição CSD1. Portanto, seja $\phi$ uma sentença proposicional e suponha que $\phi$ seja $\left\langle S_{3}, i\right\rangle$-Kripke-insatisfatível. Isso significa, por CSD1, que $V(w)(\phi)=0$ e $i \geq 0$. Portanto, como $j>i$, conclui-se que $V(w)(\phi)=0$ e $j \geq 0$, que significa que $\phi$ é $\left\langle S_{3}, j\right\rangle$-Kripke-insatisfatível. Suponha agora que $\phi$ seja $\left\langle S_{1}, i\right\rangle$-Kripke-satisfatível. Então, pela condição CSD1 para $\left\langle S_{1}, i\right\rangle$-aproximações, $V(w)(\phi)=1$ e $i \geq 0$. Portanto, como $j>i$, concluise que $V(w)(\phi)=1$ e $j \geq 0$, que significa que $\phi$ é $\left\langle S_{1}, j\right\rangle$-Kripke-satisfatível.

Outro resultado é dado pelo

Teorema 3.16 (Convergência) Se $\phi$ for 2-Kripke-satisfativel, então existe um $S \subseteq A$ e um $i \leq n+1$ tais que $\phi$ é $\left\langle S_{1}, i\right\rangle$-Kripke-satisfativel. Se $\phi$ for 2-Kripke-insatisfativel, então existe um $S \subseteq A$ e um $i \leq n+1$ tais que $\phi e ́$ $\left\langle S_{3}, i\right\rangle$-Kripke-insatisfativel.

De fato, basta tomar $S=A$ e $i \geq n$, pois então $\left\langle S_{1}, i\right\rangle$-Kripke-satisfatibilidade na sentença $\phi$ é idêntica a 2-Kripke-satisfatibilidade, e similarmente para $\left\langle S_{3}, i\right\rangle$ Kripke-insatisfatibilidade.

A idéia é que, para resolver problemas de satisfatibilidade nos diversos sistemas modais, há duas formas de aproximação: uma tenta controlar a acurácia de cada mundo no sistema modal - e isso corresponde a determinar um conjunto $S$ de proposições relevantes - e outra tenta controlar a complexidade do sistema modal, por meio de restrições sobre sua capacidade introspectiva - e isso corresponde a fixar um $i$. A primeira resolve o problema da onisciência lógica e a segunda resolve o problema da introspeç̧ão ilimitada. Esta última questão é analisada com mais detalhes no Capítulo 4 . 


\section{Capítulo 4}

\section{O Método de Massacci}

Massacci, em sua tese de doutorado [Mas98a], desenvolveu uma extensão de Cadoli-Schaerf para sentenças proposicionais quaisquer. Seu método tem forte apelo computacional e utiliza sentenças assinaladas inclusive na semântica. Adicionalmente, seu trabalho fornece uma teoria da prova a partir de SST.

Em adição ao problema da onisciência lógica, discutida nos capítulos anteriores, as lógicas modais possuem o que se convencionou chamar de introspecção ilimitada. Um agente pensante dotado dessa capacidade pode raciocinar sobre as crenças das crenças das crenças ... das crenças de alguém.

Uma forma de controlar a capacidade de instrospecção, limitando-a, é determinando um limite do número de operadores modais no escopo de outros, como foi feito por Cadoli e Schaerf e exposto na Seção 3.3. Com mais recursos de tempo e espaço, pode-se aumentar esse limite. Quanto maior o limite, mais a lógica aproximada fica próxima da Lógica Modal Clássica e da sua capacidade de introspecção ilimitada.

Uma outra idéia, devida a Massacci, sugere que o controle não seja feito de maneira estritamente sintática, limitando a profundidade modal de sentenças (como é feito por Cadoli e Schaerf), mas de maneira semântica, determinando um subconjunto do conjunto de mundos possíveis $Y \subseteq W$. Intuitivamente, esse subconjunto representa os mundos possíveis que são "interessantes", que não são muito remotos do "mundo real" e que podem ser atingidos a partir do mundo real com a utilização de apenas alguns passos introspectivos.

Neste capítulo serão apresentados, de forma adaptada, alguns resultados do trabalho de Massacci. Massacci desenvolveu seu trabalho para lógicas multimodais, em que há uma família de operadores $\left\{\square_{a}, \diamond_{a}\right\}_{a \in \mathcal{A}}$ que interagem. Aqui, por simplicidade e para facilitar a comparação entre os métodos, seus resultados em lógicas modais serão apresentados apenas para lógicas monomodais (com apenas um par de operadores $\square$ e $\diamond$ ). 


\subsection{Tableaux Proposicionais Aproximados}

Seja $A$ o conjunto de todos os átomos. Particione o conjunto $A$ em três conjuntos: o conjunto de proposições interessantes, denotado por Int $(A)$, o conjunto de proposições incoerentes, denotado por $\operatorname{Inc}(A)$ e o conjunto de proposições desconhecidas, denotado por $U n k(A)$. Assim, Int $(A) \cup \operatorname{Inc}(A) \cup U n k(A)=A$ e $\operatorname{Int}(A) \cap \operatorname{Inc}(A) \cap U n k(A)=\emptyset$. Essa partição de $A$ é chamada partição aproximada.

Intuitivamente, os átomos em $\operatorname{Int}(A)$ descrevem propriedades "interessantes" e que merecem uma interpretação clássica. Os átomos em $\operatorname{Inc}(A)$ descrevem propriedades "incoerentes", que não contribuem para provas "interessantes". Por fim, os átomos em $U n k(A)$ descrevem propriedades "desconhecidas", que não especificam propriedades de modelos "interessantes" [Mas98b].

Massacci utilizou, em todo seu trabalho, sentenças assinaladas, e o primeiro passo é determinar a valoração de sentenças assinaladas quaisquer. Seja $V$ uma 2 -interpretação no conjunto $A$. A função $V$ é estendida para sentenças assinaladas quaisquer como segue (adaptado de [Mas98a]; aqui, utiliza-se a notação unificadora de Smullyan, dada na Tabela 2.1):

1. $V(T p)=1$ sse ou $p \in \operatorname{Int}(A)$ e $V(p)=1$

$$
\begin{array}{ll}
V(F p)=1 \text { sse } & \text { ou } p \in \operatorname{Int}(A) \text { e } V(p)=0 \\
& \text { ou } p \in \operatorname{Inc}(A)
\end{array}
$$

2. $V($ neg $)=1$ sse $V($ pos $)=1$

3. $V(\alpha)=1$ sse $V\left(\alpha_{1}\right)=V\left(\alpha_{2}\right)=1$

4. $V(\beta)=1$ sse $V\left(\beta_{1}\right)=1$ ou $V\left(\beta_{2}\right)=1$

A função $V$ pode ser chamada de modelo para as sentenças assinaladas $\varphi$ tais que $V \models \varphi$. Uma função $V$ é um contra-modelo para uma sentença assinalada $\varphi$ se $V \models \bar{\varphi}$, lembrando que $\bar{\varphi}$ é a conjugada de $\varphi$. Se $\Gamma$ é um conjunto de sentenças assinaladas, $V$ é um modelo de $\Gamma$ se $V$ for um modelo para cada sentença em $\Gamma$.

A partir de uma partição de $A$ em proposições interessantes, incoerentes e desconhecidas, como se julgam os significados das sentenças compostas pelos átomos em $A$ ? Para sua apresentação, Massacci modifica um pouco a lógica 4valorada de Belnap [AB75, Bel77]. Na lógica de Belnap, todo átomo de $A$ pode assumir os valores 0,1 , inc ("incoerência") e unk ("desconhecido"). Para Massacci, átomos em $\operatorname{Int}(A)$ podem assumir apenas o valor int (ou seja, um valor "interessante", que substitui os valores 0, "falso", e 1, "verdadeiro"). Átomos em $U n k(A)$ assumem apenas o valor inc. Por fim, átomos em $U n k(A)$ assumem apenas o valor unk.

A associação dos valores int, inc e unk a átomos assinalados é simples. Se o átomo $p$ tem o valor int, então $T p$ e $F p$ têm o valor int. Se $p$ tem o valor inc, então $T p$ e $F p$ têm o valor inc. Por fim, se $p$ tem o valor unk, então $T p$ e $F p$ têm o valor unk. 
Com isso, é possível julgar sentenças assinaladas quaisquer, ou seja, determinar seu significado superficial. A Tabela 4.1 mostra como esses valores são transmitidos para sentenças mais complexas ( $X$ é qualquer um dos valores int, inc e unk). A intuição por trás desses significados superficiais é a seguinte. Se uma sentença for interessante, sua negação também deve ser; se for desconhecida, o mesmo deve suceder à negação. No caso de uma sentença do tipo $\beta$, se um dos componentes for desconhecido, o resultado total deve ser dado pelo significado do outro componente; no caso de uma sentença do tipo $\alpha$, o fato de um componente ser desconhecido se propaga para toda a sentença; e assim por diante.

\begin{tabular}{|ccc|}
\hline$\alpha_{1}$ & $\alpha_{2}$ & $\alpha$ \\
\hline unk & $X$ & unk \\
$X$ & unk & unk \\
inc & $X$ & $X$ \\
int & int & int \\
\hline
\end{tabular}

\begin{tabular}{|cc|}
\hline pos & neg \\
\hline unk & unk \\
inc & inc \\
int & int \\
\hline
\end{tabular}

\begin{tabular}{|ccc|}
\hline$\beta_{1}$ & $\beta_{2}$ & $\beta$ \\
\hline unk & $X$ & $X$ \\
inc & $X$ & inc \\
$X$ & inc & inc \\
int & int & int \\
\hline
\end{tabular}

Tabela 4.1: Significado superficial das sentenças proposicionais

A noção de conseqüência lógica aproximada é dada pela

Definição 4.1 ([Mas98a]) Dada uma partição aproximada, uma sentença $\phi$ é uma conseqüência lógica aproximada de um conjunto de sentenças ou hipóteses $\Gamma$ sse todo modelo para $\Gamma$ não for um contra-modelo para $\phi$.

O teorema a seguir, de [Mas98a], mostra que as proposições interessantes se comportam classicamente.

Teorema 4.2 Seja $\Gamma$ um conjunto de sentenças e $\phi$ uma sentença tais que todos os átomos que aparecem seja em $\Gamma$, seja em $\phi$, estejam contidos em Int $(A)$. Então, $\phi$ é uma conseqüência lógica aproximada de $\Gamma$ sse $\phi$ for uma conseqüência lógica clássica de $\Gamma$.

O comportamento de sentenças incoerentes ou desconhecidas não é clássico, como mostram os dois teoremas abaixo, também de [Mas98a]:

Teorema 4.3 Se a sentença $\phi$ for incoerente, então todo modelo satisfaz $\phi$.

Teorema 4.4 Se a sentença $\phi$ for desconhecida, então nenhum modelo satisfaz $\phi$.

Entretanto, continua valendo o Teorema da Dedução [Mas98a]:

Teorema 4.5 (Dedução) Seja $\Gamma$ um conjunto de sentenças e $\phi$ uma sentença. Uma sentença $\psi$ é uma conseqüência lógica aproximada de $\Gamma \cup\{\phi\}$ sse $\phi \supset \psi$ for conseqüência lógica aproximada de $\Gamma$.

O exemplo a seguir, adaptado de Massacci [Mas98a], ajuda a entender esse formalismo. Seja: 


$$
\begin{aligned}
& \text { - } \Gamma=\{p, p \supset q, q \supset r, r \supset s\} \\
& \text { - } \operatorname{Int}(A)=\{p, q, s\}, \operatorname{Inc}(A)=\{r\} \text { e } U n k(A)=\emptyset
\end{aligned}
$$

Então $\Gamma \vdash q$. De fato, como $p \in \operatorname{Int}(A)$, tem-se $V(T p)=1$ sse $V(p)=1$. Da mesma forma, $V(T p \supset q)=1$ sse $V(F p)=1$ ou $V(T q)=1$, o que ocorre sse $V(p)=0$ ou - uma vez que $q \in \operatorname{Int}(A)-V(q)=1$. Como $V(p)=$ 1 , tem-se $V(q)=1$, o que implica ser necessariamente $V(F q)=0-$ assim, não há modelos de $\Gamma$ que sejam contra-modelos de $q$. Por outro lado $r$ não é conseqüência lógica aproximada de $\Gamma$. Isso porque, $\operatorname{como} r \in \operatorname{Inc}(A)$, pode ocorrer $V(T p)=1, V(T p \supset q)=1$ e $V(T q \supset r)=1$ mas, simultaneamente, $V(F r)=1$. De fato, as três primeiras igualdades implicam, respectivamente, que $V(p)=1$ (pois $p \in \operatorname{Int}(A)), V(F p)=1$ ou $V(T q)=1-\mathrm{e}$ isto significa que $V(p)=0$ ou $V(q)=1$ (pois $q \in \operatorname{Int}(A))-$, e $V(F q)=1$ ou $V(\operatorname{Tr})=1$. Como $V(p)=0$ ou $V(q)=1$ e $V(p)=1$, necessariamente $V(q)=1$, ou seja, $V(F q)=$ 0 , e isso implica que $V(T r)=1$. Mas como $r \in \operatorname{Inc}(A)$, simultâneamente $V(T r)=V(F r)=1$. Em particular, $V(F r)=1$, o que mostra um contramodelo ao seqüente $\Gamma \vdash r$.

Tableaux Proposicionais Aproximados possuem algumas mudanças das regras de redução dos Tableaux de Smullyan segundo o significado superficial da sentença assinalada sobre a qual se deseja aplicar a regra:

R1. se o significado superficial de $\varphi$ for int, então $\varphi$ é reduzida de acordo com a definição clássica

R2. se o significado superficial de $\varphi$ for inc, então $\varphi$ já está reduzida - isso é o mesmo que dizer que, quando a sentença $\varphi$ for incoerente, não é possível aplicar regras de tableau a ela

R3. se o significado superficial de $\varphi$ for unk, então o ramo do tableau fecha

As últimas duas regras acima são regras não clássicas de redução ou contradição, respectivamente. Por essas regras, pode-se ver que o conjunto $\operatorname{Inc}(A)$ funciona para aproximar a lógica por baixo, enquanto o conjunto $U n k(A)$ funciona para aproximar a lógica por cima. Isso será analisado com mais detalhes adiante, ao mostrar-se que o método de Massacci generaliza Cadoli-Schaerf, contendo este último como caso particular.

O exemplo a seguir, adaptado de [Mas98a], ilustra um tableau proposicional aproximado (Figura 4.1) que tenta provar o exemplo dado acima, ou seja, que $s$ é conseqüência lógica de $\Gamma$, em que

$$
\begin{aligned}
& \text { - } \Gamma=\{p, p \supset q, q \supset r, r \supset s\} \\
& \text { - } \operatorname{Int}(A)=\{p, q, s\}, \operatorname{Inc}(A)=\{r\} \text { e } U n k(A)=\emptyset
\end{aligned}
$$

As sentenças (6) e (7) são obtidas de (2) por aplicação de $\beta$. Esse tableau está reduzido (pois, como $r \in \operatorname{Inc}(A)$, os significados de $T q \supset r$ e $\operatorname{Tr} \supset s$ são incoerentes), mas não fechou. O exemplo sugere um algoritmo para fazer uma dedução proposicional. Por prova clássica ou contra-modelo clássico entende-se uma prova ou contra-modelo que não utiliza as regras não clássicas. 


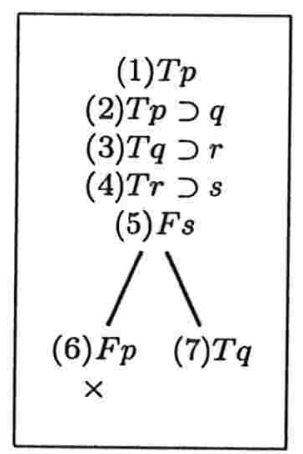

Figura 4.1: Um exemplo de aplicação dos Tableaux Proposicionais Aproximados

1. Escolha uma partição aproximada de forma que o conjunto de átomos interessantes em $\operatorname{Int}(A)$ seja pequeno

2. compute um tableau aproximado

3. se o tableau aproximado for uma prova clássica ou um contra-modelo clássico, então pare

4. senão, encontre a parte do tableau onde regras não clássicas de redução ou fechamento são usadas, aumente o conjunto de átomos interessantes, colocando nele pelo menos um átomo que aparece em uma sentença sob a qual essas regras não clássicas são usadas, e volte para o segundo passo

Lembrando das definições da Seção 2.1.2, diz-se que um determinado tableau é correto com relação a determinada semântica se toda inferência realizada por meio do tableau pode ser feita também pela semântica; um tableau é completo com relação a determinada semântica se toda inferência realizada pela semântica pode ser feita também por meio do tableau. Massacci provou, em [Mas98a], que seu tableau de prova é correto e completo com relação à sua semântica:

Teorema 4.6 (Correção) Dada uma partição aproximada, se uma sentença $\phi$ tem uma prova por Tableaux Aproximados com hipóteses $\Gamma$, então ela é uma conseqüência lógica aproximada de $\Gamma$.

Teorema 4.7 (Completude) Dada uma partição aproximada, se uma sentença $\phi$ é uma conseqüência lógica aproximada de um conjunto de hipóteses $\Gamma$, então ela tem uma prova por Tableaux Aproximados com $\Gamma$.

Para relacionar seus conceitos aos de Cadoli-Schaerf, Massacci precisou adaptar o sistema de Cadoli-Schaerf a sentenças assinaladas. Assim, ele introduz as seguintes definições [Mas98a]:

Definição 4.8 Uma $S_{1}$-interpretação assinalada é uma valoração $V$ de átomos assinalados, de forma que $V(T p) \neq V(F p)$ se $p \in S$ e $V(T p)=V(F p)=0$ se $p \in A \backslash S$. 
Definição 4.9 Uma $S_{3}$-interpretação assinalada é uma valoração $V$ de átomos assinalados, de forma que $V(T p) \neq V(F p)$ se $p \in S$ e não ocorre simultaneamente que $V(T p)=V(F p)=0$ se $p \in A \backslash S$.

A extensão da função $V$ para outras sentenças é feita utilizando as condições 2-4 dadas no começo desta seção. As noções de $S_{1}$ e $S_{3}$-inferência são similares às da Seção 3.1, considerando as adaptações evidentes para sentenças assinaladas. Naturalmente, a equivalência de sistemas só ocorre se forem consideradas sentenças em CNF, pois Cadoli e Schaerf trabalharam apenas com elas. Massacci provou, em [Mas98a], os seguintes teoremas:

Teorema 4.10 Seja $\Gamma \cup\{\phi\}$ um conjunto de sentenças em CNF e seja $S \subseteq A$. Seja Int $(A)=S$ e Unk $(A)=\emptyset$ (portanto, $\operatorname{Inc}(A)=A \backslash S)$. Então $\Gamma \models_{S}^{3} \phi$ sse $\phi$ é uma conseqüência lógica aproximada de $\Gamma$.

Teorema 4.11 Seja $\Gamma \cup\{\phi\}$ um conjunto de sentenças em $C N F$ e seja $S \subseteq A$. Seja $\operatorname{Int}(A)=S$ e Inc $(A)=\emptyset$ (portanto, $\operatorname{Unk}(A)=A \backslash S)$. Então $\Gamma \models_{S}^{1} \phi$ sse $\phi$ é uma conseqüência lógica aproximada de $\Gamma$.

Assim, o conjunto de proposições incoerentes está relacionado à $S_{3}$-interpretação de Cadoli-Schaerf, no sentido que proposições "fora" de $S$ são incoerentes, podem assumir qualquer valor. O conjunto de proposições desconhecidas está relacionado à $S_{1}$-interpretação, no sentido que proposições "fora" de $S$ assumem o valor falso por serem desconhecidas.

\subsection{Tableaux Modais Aproximados}

Massacci apresentou, em [Mas98b, Mas98a], uma extensão de seu procedimento para lógicas modais.

A noção de contra-modelo é similar à da seção 4.1. A verdade em sentenças assinaladas (modais ou não) é determinada pelas condições abaixo $(w \in W)$ :

1. $\mathcal{M}, w \models T p$ sse $V(w)(p)=1$ $\mathcal{M}, w \models F p$ sse $V(w)(p)=0$

2. $\mathcal{M}, w \models T \neg \phi$ sse $\mathcal{M}, w \models F \phi$ $\mathcal{M}, w \models F \neg \phi$ sse $\mathcal{M}, w \models T \phi$

3. $\mathcal{M}, w \models T(\phi \wedge \psi)$ sse $\mathcal{M}, w \models T \phi$ e $\mathcal{M}, w \models F \psi$ $\mathcal{M}, w \models F(\phi \wedge \psi)$ sse $\mathcal{M}, w \models F \phi$ ou $\mathcal{M}, w \models F \psi$

4. $\mathcal{M}, w \models T(\phi \vee \psi)$ sse $\mathcal{M}, w \models T \phi$ ou $\mathcal{M}, w \models F \psi$ $\mathcal{M}, w \models F(\phi \vee \psi)$ sse $\mathcal{M}, w \models F \phi$ e $\mathcal{M}, w \models F \psi$

5. $\mathcal{M}, w \models T \square \phi$ sse para todo $t \in W$ tal que $w R t, \mathcal{M}, w \models T \phi$ $\mathcal{M}, w \models F \square \phi$ sse existe $t \in W$ tal que $w R t$ e $\mathcal{M}, w \models T \phi$ 
A relação dessa semântica com a dada na Seção 4.1 é a seguinte. Considera-se o conjunto de átomos $L$ particionado em $\operatorname{Int}(L), \operatorname{Inc}(L)$ e $U n k(L)$. Uma aproximação unicamente proposicional modifica a cláusula 1 acima para [Mas98b]

$$
\begin{aligned}
1^{\prime} . \quad \mathcal{M}, w \models T p \text { sse } & \text { ou } p \in \operatorname{Int}(L) \text { e } V(w)(p)=1 \\
& \text { ou } p \in \operatorname{Inc}(L) \\
\mathcal{M}, w \models F p \text { sse } & \text { ou } p \in \operatorname{Int}(L) \text { e } V(w)(p)=0 \\
& \text { ou } p \in \operatorname{Inc}(L)
\end{aligned}
$$

As outras cláusulas 2-5 permanecem iguais.

Fixado um mundo $w \in W$, os resultados da Seção 4.1 para sentenças proposicionais continuam valendo, e pelas mesmas razões.

Massacci também introduziu [Mas98b] uma distinção entre os mundos envolvidos em um modelo de lógica modal, fazendo uma partição e dividindo o conjunto de mundos possíveis $W$ em três: mundos interessantes $\operatorname{Int}(W)$, mundos incoerentes $\operatorname{Inc}(W)$ e mundos desconhecidos $U n k(W)$. Dessa forma,tem-se que $W=\operatorname{Int}(W) \cup \operatorname{Inc}(W) \cup U n k(W)$ e $\operatorname{Int}(W) \cap \operatorname{Inc}(W) \cap U n k(W)=\emptyset$. Intuitivamente, mundos interessantes não devem ser "muito remotos" do mundo "real", ou seja, devem ser alcançáveis com poucos passos de introspeção. A avaliação de mundos "muito distantes" vai depender do fato de ser o agente crédulo (mundos incoerentes) ou cético (mundos desconhecidos).

Em mundos incoerentes, todas as afirmações são aceitas, inclusive as contraditórias. Nos mundos desconhecidos, por outro lado, nenhuma afirmação é admitida.

Primeiro, há uma restrição sobre as relações de acessibilidade $R_{a}$. Se $w R t$ e $w \in \operatorname{Inc}(L)$, então $t \in \operatorname{Inc}(L)$; se $w R t$ e $w \in U n k(L)$, então $t \in U n k(L)$. Uma aproximação unicamente modal modifica a cláusula 1 para

$$
\begin{aligned}
1^{\prime \prime} . \mathcal{M}, w \models T p \text { sse } & \text { ou } w \in \operatorname{Int}(W) \text { e } V(w)(p)=1 \\
& \text { ou } w \in \operatorname{Inc}(W) \\
\mathcal{M}, w \models F p \text { sse } & \text { ou } w \in \operatorname{Int}(W) \text { e } V(w)(p)=0 \\
& \text { ou } w \in \operatorname{Inc}(W)
\end{aligned}
$$

Para que se possa ter um controle fino do poder de introspecção, Massacci desenvolveu [Mas98b] o conceito de mundos realistas. Inicialmente, ele define um agente limitado como sendo uma dupla $\left\langle k_{m}, x\right\rangle$, em que $k_{m}$ é um número inteiro não negativo (o número máximo de passos de introspecção, ou limite introspectivo) e $x$ pode tomar os valores $c$ para crédulo (dando origem aos agentes $k_{m}$-crédulos) e $s$ para cético (dando origem aos agentes $k_{m}$-céticos). Se $\sigma$ tem um limite introspectivo $k_{m}$, então restam apenas outros $k_{m}-|\sigma|$ passos para o agente pensante. Segue-se a definição:

Definição 4.12 Seja $\left\langle k_{m}, x\right\rangle$ um agente limitado e um modelo $\mathcal{M}$ um modelo com uma partição dos mundos. Um mundo $w \in W$ é $k_{m}$-realista se $w \in \operatorname{Int}(W)$ e se:

- $k_{m}=0$ e $x=s$, então wRt implica que $v \in \operatorname{Inc}(W)$

- $k_{m}=0$ e $x=c$, então wRt implica que $v \in U n k(W)$ 
- $k_{m}>1$, então wRt implica que $v$ é $\left(k_{m}-1\right)$-realista.

Para inferências realizadas com um agente limitado, utiliza-se a relação $\models^{k_{m}}$, $x$.

Um exemplo desse conceito, de [Mas98b], é o seguinte. Considere um agente 1-cético. Então

$$
\models^{1, s} \square(p \supset q) \wedge \square p \supset \square q
$$

Isso vale porque, para um agente 1-cético, o primeiro passo de introspeç̧ão se comporta como no caso clássico. Entretanto, ocorre

$$
\not^{1, s} \square \square(p \supset q) \wedge \square \square p \supset \square \square q
$$

Isso porque, no segundo passo da introspecção de um agente 1-cético, todos os mundos ligados são necessariamente incoerentes, e mundos incoerentes fornecem um contra-modelo (bem como um modelo) para a sentença em questão. Essa é uma das razões que o conceito de conseqüência lógica aproximada é dada na forma da Definição 4.1: podem existir mundos que dão simultaneamente modelos e contra-modelos.

Com esses conceitos em mão, é possível julgar sentenças assinaladas quaisquer, ou seja, determinar seu significado superficial. Como na Seção 4.1, isso é importante para desenvolver um tableau de prova. Neste caso, é útil considerar que o significado superficial de uma sentença assinalada é uma função $V(k, \varphi)$ de dois argumentos, que toma valores no conjunto $\{$ int, inc, unk $\}$. O primeiro argumento é o grau de introspeç̧ão (um número inteiro não negativo) e o segundo é a sentença assinalada.

Para átomos assinalados, tem-se que, para todo grau de introspecção $k$ que

$$
\begin{aligned}
& V(k, T p) \\
& V(k, F p)
\end{aligned}=\left\{\begin{array}{l}
\text { int se } p \in \operatorname{Int}(L) \\
\text { inc se } p \in \operatorname{Inc}(L) \\
\text { unk se } p \in \operatorname{Unk}(L)
\end{array}\right.
$$

A extensão da função $V$ para conectivos proposicionais e modais é feita por indução. Para os proposicionais, refere-se à Tabela 4.1 da Seção 4.1. A extensão para os conectivos modais é dada na Tabela 4.2.

Se o limite introspectivo não está especificado, define-se o significado da sentença (modal) como:

$$
\begin{aligned}
& V(*, \nu)=V\left(k_{m}, \nu\right) \\
& V(*, \pi)=V\left(k_{m}, \pi\right)
\end{aligned}
$$

É possível uma combinação dos dois tipos de aproximação, fazendo uma partição do conjunto de mundos possíveis $W$ e associando, a cada $w \in W$, uma partição possivelmente distinta do conjunto de átomos $L$. Qual das duas partições seria dominante na determinação da verdade? Em [Mas98b, Mas98a], Massacci sugeriu uma aproximação combinada. Para simplificar, ele assume que a cada mundo $w$ associa-se sempre a mesma partição de $L$. 


$$
\begin{aligned}
V(0, \pi) & =\left\{\begin{array}{l}
\text { inc se o agente for cético } \\
\text { unk se o agente for crédulo }
\end{array}\right. \\
V(k+1, \pi) & =V\left(k, \pi_{0}\right) \\
V(0, \nu) & =\left\{\begin{array}{l}
\text { inc se o agente for cético } \\
\text { int se o agente for crédulo }
\end{array}\right. \\
V(k+1, \nu) & =\left\{\begin{array}{l}
\text { inc se } V\left(k, \nu_{0}\right)=i n c \\
\text { int nos outros casos }
\end{array}\right.
\end{aligned}
$$

Tabela 4.2: Significado superficial das sentenças modais

$$
\begin{array}{ll}
\text { MS'"'1. }^{\prime \prime}, w \models T p \text { sse } & \text { ou } w \in \operatorname{Int}(W) \text { e } p \in \operatorname{Int}(L) \text { e } V(w)(p)=1 \\
& \text { ou } w \in \operatorname{Int}(W) \text { e } p \in \operatorname{Inc}(L) \\
& \text { ou } p \in \operatorname{Inc}(W) \\
\mathcal{M}, w \models F p \text { sse } & \text { ou } w \in \operatorname{Int}(W) \text { e } p \in \operatorname{Int}(L) \text { e } V(w)(p)=0 \\
& \text { ou } w \in \operatorname{Int}(W) \text { e } p \in \operatorname{Inc}(L) \\
& \text { ou } p \in \operatorname{Inc}(W)
\end{array}
$$

Tableaux Modais Aproximados têm algumas mudanças nas regras de redução dos SST segundo o significado superficial da sentença assinalada sobre a qual se deseja aplicar a regra [Mas98a]:

- se o significado superficial de $\sigma: \varphi$ for int, então $\sigma: \varphi$ é reduzida de acordo com a definição clássica

- se $\sigma=1$ e $V(*, \varphi)=i n c$, então $\sigma: \varphi$ já está reduzida

- se $V\left(k_{m}-|\sigma|, \varphi\right)=i n c$, então $\sigma: \varphi$ já está reduzida

- se $\sigma=1$ e $V(*, \varphi)=u n k$, então o ramo do tableau fecha

- se $\left.V\left(k_{m}-|\sigma|, \varphi\right)=u n k\right)$, então o ramo do tableau fecha

As últimas quatro regras acima são regras não clássicas de redução ou contradição. $\mathrm{O}$ algoritmo sugerido pelas regras de Tableaux Modais Aproximados é dado abaixo [Mas98a]:

1. Escolha uma partição aproximada de forma que o conjunto de átomos interessantes em $\operatorname{Int}(L)$ seja pequeno

2. escolha um limite máximo de introspecção $k_{m}$ pequeno, e decida se $x=c$ ou $s$

3. compute um tableau aproximado

4. se o tableau aproximado for uma prova clássica ou um contra-modelo clássico, então pare 
5. senão, encontre a parte do tableau onde regras não clássicas de redução ou fechamento são usadas e mude esse status:

- seja aumentando o conjunto de átomos interessantes, colocando nele pelo menos um átomo que aparece em uma sentença sob a qual essas regras não clássicas são usadas

- seja aumentando o limite $k_{m}$

volte para o terceiro passo

Por fim, Massacci [Mas98a] demontra a correção e completude dos Tableaux Modais Aproximados com relação à semântica dada nesta seção, assim como fez para o caso proposicional.

Teorema 4.13 (Correção) Dada uma partição aproximada, se uma sentença $\phi$ tem uma prova por Tableaux Aproximados com hipóteses $\Gamma$, então ela é uma conseqüência lógica aproximada de $\Gamma$.

Teorema 4.14 (Completude) Dada uma partição aproximada, se uma sentença $\phi$ é uma conseqüência lógica aproximada de um conjunto de hipóteses $\Gamma$, então ela tem uma prova por Tableaux Aproximados com $\Gamma$. 


\section{Capítulo 5}

\section{O Método de Finger-Wassermann}

Marcelo Finger e Renata Wassermann procuraram estender o método de CadoliSchaerf (veja, por exemplo, [FW01, FW02]). Aqui, serão analisado apenas seus trabalhos de extensão para aproximações por baixo, pelas razões explicitadas no Capítulo 3. Em [FW01], eles apresentam duas limitações principais a CadoliSchaerf:

1. O sistema é restrito a sentenças sem o operador $\supset$ e em NNF. Sob semânticas não clássicas, como analisado em [CPW00], a tradução usual de sentenças para forma clausal não preserva valores verdade.

2. O conjunto $S$ precisa ser adivinhado em cada passo da aproximação; nenhum método é dado para determinar os átomos a serem adicionados a $S$.

Essas duas limitações são enfrentadas por meio de uma nova semântica que não mais restringe as sentenças sem o operador $\supset$ e em NNF, o que resolve o primeiro problema, e com a apresentação de um tableau para inferência aproximada, o que resolve o segundo. Neste capítulo, serão descritos esses dois trabalhos.

\subsection{Semântica e Principais Propriedades}

A semântica de Finger-Wassermann é uma extensão da semântica de CadoliSchaerf. Seja $A$ o conjunto de todos os átomos e $S \subseteq A$ um subconjunto de proposições relevantes. Para uma sentença NNF, tem-se que a valoração $S_{3}$ definida abaixo associa os mesmos valores verdade que a $S_{3}$-interpretação de Cadoli-Schaerf, definida na Seção 3.1. No que segue, assume-se a existência de uma valoração dos átomos. Utiliza-se uma convenção muito conveniente: dada uma sentença $\phi$, o fato de que todos os átomos de $\phi$ estão em $S$ é denotado por $\phi \in S$. 
Seja $\mathcal{L}_{C}$ o conjunto de todas as sentenças da linguagem. Uma valoração $S_{3}$ é uma função $v_{S}^{3}: \mathcal{L}_{C} \rightarrow\{0,1\}$ que estende uma valoração total nos átomos de $A$ como segue:

FW1. $v_{S}^{3}(\phi \wedge \psi)=1$ sse $v_{S}^{3}(\phi)=v_{S}^{3}(\psi)=1$

FW2. $v_{S}^{3}(\phi \vee \psi)=0$ sse $v_{S}^{3}(\phi)=v_{S}^{3}(\psi)=0$

FW3. $v_{S}^{3}(\phi \supset \psi)=0$ sse $v_{S}^{3}(\phi)=1$ e $v_{S}^{3}(\psi)=0$

FW4. quando $\phi \in S, v_{S}^{3}(\neg \phi)=0$ sse $v_{S}^{3}(\phi)=1$

FW5. não acontece simultaneamente que $v_{S}^{3}(\phi)=v_{S}^{3}(\neg \phi)=0$

A cláusula FW5 definida acima não é a mesma de [FW01], mas lhe é equivalente. A razão dessa formulação é que, para o autor, ela parece mais intuitiva e mais fiel à definição original de Cadoli-Schaerf. É imediato que a semântica é paraconsistente fora de $S$, ou seja, pode ocorrer que tanto $\phi$ como $\neg \phi$ sejam verdadeiras. Dentro de $S$, o comportamento das sentenças é clássico. Fora de $S$, todos os operadores com exceção de $\neg$ continuam se comportando classicamente. Da maneira padrão, define-se $S_{3}$-satisfatibilidade, $S_{3}$-validade e $S_{3}$-insatisfatibilidade ${ }^{1}$, representados usando a relação $\models_{S}^{3}$.

A valoração $v_{S}^{3}$ não é determinada unicamente pela valoração nos átomos. Uma pergunta natural que segue disso é: o que a determina? O teorema abaixo, refraseado de $[\mathrm{FW} 01]^{2}$, dá a resposta.

Teorema 5.1 A valoração $v_{S}^{3}$ é determinada completamente por seu valor no conjunto $A \cup\left\{\neg \phi \mid \phi \notin S\right.$ e $\left.v_{S}^{3}(\phi)=1\right\}$.

Dem: Para os conectivos $\wedge, \vee$ e $\supset$, é imediato que o valor de $v_{S}^{3}$ é determinado pelo das subsentenças. Se $\phi \in S$, então a cláusula FW4 implica que o valor de $\neg \phi$ é oposto ao de $\phi$. Suponha, então, que $\phi \notin S$. Se $v_{S}^{3}(\phi)=0$, a cláusula FW5 implica que $v_{S}^{3}(\neg \phi)=1$. Restam apenas as sentenças em $A \cup\{\neg \phi \mid \phi \notin$ $S$ e $v_{S}^{3}(\phi)=1$ \} para determinar $v_{S}^{3}$.

Esse teorema sugere que é possível interpretar uma sentença em $S_{3}$ por meio de outra sentença clássica que resulte da adição a $A$ de novos átomos. De fato isso é possível, e torna muito mais fácil intuir sobre $S_{3}$. Seja $p_{\neg \phi}$ um novo átomo (para cada $\phi \notin S$ tal que $v_{S}^{3}(\phi)=0$ ) cuja valoração é igual a $v_{S}^{3}(\neg \phi)$, e defina a tradução $\phi^{\bullet S}$ de $\phi$ da seguinte maneira:

$$
\begin{aligned}
p^{\bullet S} & =p \\
(\phi \wedge \psi)^{\bullet S} & =\phi^{\bullet S} \wedge \psi^{\bullet S} \\
(\phi \vee \psi)^{\bullet S} & =\phi^{\bullet S} \vee \psi^{\bullet S} \\
(\phi \supset \psi)^{\bullet S} & =\phi^{\bullet S} \supset \psi^{\bullet S}
\end{aligned}
$$

\footnotetext{
${ }^{1}$ De fato, são similares às dadas no método de Cadoli-Schaerf. A única diferença é que, neste caso, a semântica atende a uma linguagem mais rica. Por isso, nesta seção utiliza-se as mesmas notações do Capítulo 3 embora, estritamente falando, sejam dois sistemas distintos.

${ }^{2} \mathrm{Em}$ [FW01], afirma-se que o conjunto determinante é $A \cup\{\neg \phi \mid \phi \notin S\}$. A formulação dada aqui diminui esse conjunto.
} 


$$
(\neg \phi)^{\bullet S}= \begin{cases}\neg\left(\phi^{\bullet S}\right) & \text { se } \phi \in S \text { ou se } v_{S}^{3}(\phi)=0 \\ p_{\neg \phi} & \text { se } \phi \notin S \text { e } v_{S}^{3}(\phi)=1\end{cases}
$$

A idéia da tradução de sentenças $\neg \phi$ é que, dentro de $S$, elas recebam a mesma valoração. Fora de $S$, deve-se traduzir $\neg \phi$ de tal forma que não possa ocorrer simultaneamente que ambas $\phi^{\bullet S}$ e $(\neg \phi)^{\bullet S}$ recebam o valor falso. Há liberdade de escolha somente quando $v_{S}^{3}(\phi)=1$, e é por isso que apenas nesse caso é preciso associar a $\phi$ um novo átomo.

As vantagens da tradução de uma sentença aparecem no teorema a seguir, de [FW01], que permite determinar $S_{3}$-satisfatibilidade de uma sentença a partir de sua tradução.

Teorema 5.2 Uma sentença $\phi$ é $S_{3}$-satisfativel sse $\phi^{\bullet S}$ é satisfativel classicamente.

Traduções também são empregadas na demonstração do seguinte teorema, também provado em [FW01] e que mostra uma característica importante da semântica para sentenças fora de $S$ :

Teorema 5.3 Seja Prop $(\phi)$ o conjunto dos átomos de $\phi$. Toda sentença $\phi$ com $\operatorname{Prop}(\phi) \cap S=\emptyset$ é $S_{3}$-satisfativel.

Como era de se esperar, inferências em $S_{3}$ são corretas; isso é uma consequêencia do próximo teorema, que nada mais é que uma formulação do Teorema 3.4 para a semântica extendida.

Teorema 5.4 (Monotonicidade) Seja $S \subseteq S^{\prime} \subseteq A$. Se uma sentença $\phi$ é $S_{3}$-válida, entâo é também $S_{3}^{\prime}$-válida.

Dem: Uma $S^{\prime}$-3-valoração $v_{S^{\prime}}^{3}$ deve satisfazer as condições FW1-FW5 para $S^{\prime}$. As condições FW1-FW3 e FW5 independem de $S^{\prime}$ e, portanto, $v_{S^{\prime}}^{3}$ as satisfaz como uma $S_{3}$-valoração também. Se $v_{S^{\prime}}^{3}$ satisfaz FW4 para $S^{\prime}$, então ocorrem duas coisas. Primeiro, ela satisfaz FW4 para $S$ quando $\phi \in S \subseteq S^{\prime}$. Segundo, não mapeia um par de sentenças opostas simultaneamente em 0 e, portanto, para sentenças $\phi \in S^{\prime}$ com $\phi \notin S$, $v_{S^{\prime}}^{3}$ continua satisfazendo FW5 para $S$. Assim, toda $S^{\prime}$-3-valoração é uma $S_{3}$-valoração, o que mostra o teorema.

Dessa forma, se for tomado $S^{\prime}=A$, conclui-se que se uma sentença é $S_{3}$-válida, então é válida classicamente.

Também vale, e a demonstração é trivial (tome $S=A$ ), o seguinte

Teorema 5.5 (Convergência) Se uma sentença $\phi$ é válida classicamente, então existe $S \subseteq$ A tal que $\phi$ é também $S_{3}$-válida.

Por fim, como o comportamento do operador $\supset$ é clássico, o Teorema da Dedução continua válido:

Teorema 5.6 (Dedução) Seja $\Gamma$ um conjunto de sentenças e $\phi, \psi$ duas sentenças quaisquer. Então $\Gamma \cup\{\phi\} \models_{S}^{3} \psi$ sse $\Gamma \models_{S}^{3} \phi \supset \psi$. 
No método de Cadoli-Schaerf, todas as sentenças devem estar em NNF. Portanto, a versão de modus ponens naquele método deve ser formulado sem o operador $\supset$ (de $\phi$ e $\neg \phi \vee \psi$ pode-se inferir $\psi$ ); como foi visto, essa versão de modus ponens não vale de modo geral, e isso continua ocorrendo para FingerWassermann. Mas agora é possível formular modus ponens da maneira usual, com o operador $\supset$. E nessa formulação, pelo fato da semântica do operador ser clássica, modus ponens continua valendo $\left(\operatorname{se} v_{S}^{3}(\phi)=v_{S}^{3}(\phi \supset \psi)=1\right.$, não pode ocorrer $\left.v_{S}^{3}(\psi)=0\right)$. Isso demonstra que diferentes versões de regras de inferência, que classicamente são equivalentes, não o são na semântica de FingerWassermann.

\subsection{Tableaux de Prova}

Finger e Wassermann também apresentaram uma teoria de prova para sua semântica, e mostraram a correção e completude da primeira com relação à última. Esse trabalho não foi feito por Cadoli e Schaerf. Esta seção trata dos resultados obtidos.

Como foi observado na Seção 2.1.2, Finger e Wasserman utilizam KE-tableaux para sua teoria da prova. Um KE-tableau para $S_{3}$ ou $K E S_{3}$-tableau, é obtido da Tabela 2.3 com uma pequena modificação. A regra $(T \neg)$ é mudada para

$$
\frac{T \neg \phi}{F \phi} \text { desde que } \phi \in S
$$

O significado dessa nova regra é que uma inferência com a regra $(T \neg)$ pode ser feita desde que $\phi$ esteja no conjunto das proposições relevantes; caso contrário, nada pode ser inferido.

Deve-se provar a correção e a completude do $\mathrm{KES}_{3}$-tableau com relação à semântica fornecida na Seção 5.1. Em [FW01], são demonstrados os seguintes teoremas:

Teorema 5.7 (Correção) Seja $\Gamma$ um conjunto finito de sentenças. Suponha que um tableau para o seqüente $\Gamma \vdash \psi$ feche. Então $\Gamma \models_{S}^{3} \psi$.

Teorema 5.8 (Completude) Seja $\Gamma$ um conjunto finito de sentenças. Suponha que $\Gamma \models_{S}^{3} \psi$. Então, qualquer $K E S_{3}$-tableau para $\Gamma \vdash \psi$ fecha.

$\mathrm{O}$ uso de $\mathrm{KE} S_{3}$-tableaux é especialmente útil para o desenvolvimento de um algoritmo de incrementabilidade para o conjunto $S$. Para a compreensão da origem desse algoritmo, será dado um exemplo, tirado de [FW01]. Suponha que se deseje determinar se o tableau consegue provar a equivalência entre $\phi \supset \psi$ e $\neg \phi \vee \psi$. Na Seção 5.1 , foi visto que a semântica de $S_{3}$ refuta essa equivalência. Então, pela correção e completude, o mesmo deve ocorrer com o tableau. Para mostrar essa equivalência por tableaux, é necessário que se tentem refutar os seqüentes $\phi \supset \psi \vdash \neg \phi \vee \psi \mathrm{e} \neg \phi \vee \psi \vdash \phi \supset \psi$. O primeiro seqüente não pode ser refutado, como mostra o tableau ao lado esquerdo da Figura 5.1. Nesse tableau, as sentenças (3) e (4) são obtidas de (2) pela regra $(F \vee)$; (5) é obtida de (3) por 
$(F \neg)$; e (6) é obtida de (1) e (5) por ( $\left.T \supset_{1}\right)$. Entretanto, o segundo seqüente pode ser refutado, como mostra o tableau ao lado direito da Figura 5.1. Nesse tableau, as sentenças (3) e (4) são obtidas de (2) por ( $F \supset)$. (5) é obtida de (1) e (4) por $\left(T \vee_{2}\right)$.

\begin{tabular}{|cc|}
\hline & \\
$(1) T \phi \supset \psi$ & $(1) T \neg \phi \vee \psi$ \\
$(2) F \neg \phi \vee \psi$ & $(2) F \phi \supset \psi$ \\
$(3) F \neg \phi$ & $(3) T \phi$ \\
$(4) F \psi$ & $(4) F \psi$ \\
$(5) T \phi$ & $(5) T \neg \phi$ \\
$(6) T \psi$ & $?$ \\
$\times$ & \\
\hline
\end{tabular}

Figura 5.1: Um exemplo de aplicação dos $\mathrm{KES}_{3}$-tableaux

O símbolo de interrogação na linha 5 representa o fato de que, nesse ponto, não há como fazer inferências a partir de $T \neg \phi$ sem mais informação. A inferência só seria possível se $\phi \in S$. Se, no começo, $S=\emptyset$, é preciso apenas adicionar os átomos de $\phi$ a $S$ para realizar a inferência $F \phi$ e fechar o tableau. Assim, adiciona-se átomos a $S$ conforme eles são necessários para fazer novas inferências. O seguinte algoritmo, devido a Finger e Wassermann e apresentado em [FW01], surge naturalmente.

1. $S:=\emptyset$

2. transforme o seqüente de entrada em um $\mathrm{KE} S_{3}$-tableau inicial

3. expanda o tableau até que ele feche ou fique bloqueado (como ocorreu com o tableau acima na interrogação)

4. se o tableau fechar, terminar com sucesso

5. se o tableau contiver um ramo que não puder ser classicamente expandido, terminar com falha

6. se o tableau ficar bloqueado devido a uma sentença $T \neg \phi$, faça $S:=S \cup\{\phi\}$ e volte ao passo 3

Assim, o método de Finger-Wassermann resolve os dois problemas a que se propõe resolver, conforme expostos no começo deste capítulo: estender CadoliSchaerf para sentenças quaisquer e apresentar um algoritmo para determinar os átomos a serem adicionados a $S$.

Uma pergunta cabível é: por que a semântica é enfraquecida apenas para o operador $\neg$ ? Em outro artigo, [FW04], Marcelo Finger e Renata Wassermann generalizam completamente a semântica para uma semântica denotada por $S_{e}$. Para cada operador e cada possível regra do KE-tableau, existe um conjunto de proposições relevantes associado. Por exemplo, para as regras $\left(F \wedge_{1}\right)$ e $\left(F \wedge_{2}\right)$ 
associa-se um conjunto de proposições relevantes "para a verdade do operador $\wedge$ ", $S_{\wedge}^{F}$. Para essa semântica existe um $\mathrm{KE} S_{e}$-tableau, que é uma generalização do KE-tableau de d'Agostino; em [FW04] prova-se a correção e completude do tableau com relação à semântica generalizada $S_{e}$. Os detalhes dessa generalização não entram no contexto do presente trabalho e não serão analisados.

Finger e Wassermann também buscaram, em [FW02], uma extensão das aproximações por cima (as $S_{1}$-aproximações) de Cadoli-Schaerf. As propriedades computacionais de uma tal extensão mostraram-se extremamente complexas e ainda estão sendo pesquisadas. Neste trabalho, o interesse centra-se nas aproximações por baixo (por razões apresentadas no Capítulo 3) e, por essa razão, os detalhes da extensão das $S_{1}$-aproximações de Cadoli-Schaerf não serão estudados. 


\section{Capítulo 6}

\section{Finger-Wassermann para Lógicas Modais}

Este capítulo apresenta uma extensão do trabalho de Marcelo Finger e Renata Wassermann para Lógica Modal Clássica. Na Seção 6.1, propõe-se uma semântica que modifique apenas o comportamento do operador $\neg$ e analisam-se algumas propriedades. Depois, inspirado pelo trabalho de Cadoli-Schaerf em lógicas modais, incorpora-se um controle da introspecção, introduzindo-se um limite máximo de introspecção $n$. A semântica obtida é associada a uma teoria da prova desenvolvida a partir de SST, apresentada na Seção 6.2. Ali, também são demonstrados correção e completude dos tableaux apresentados com relação a essa semântica. A Seção 6.3 apresenta uma semântica e teoria da prova alternativos, que resolvem um problema técnico surgido no desenvolvimento da semântica e teoria da prova das duas seções anteriores. Na Seção 6.4, discute-se uma generalização da semântica apresentada na Seção 6.1 e algumas propriedades interessantes que podem ser estudadas em trabalhos futuros.

\subsection{Semântica e Principais Propriedades}

Seja $S \subseteq A$ um conjunto de proposições relevantes, $W$ um conjunto de mundos possíveis, $R$ uma relação de acessibilidade e $v_{S}^{3}: W \rightarrow\left(A^{\circ} \rightarrow\{0,1\}\right)$ uma função que associa a cada $w \in W$ uma valoração $S_{3}$ total no conjunto $A^{\circ}$ dos literais. Denote por $\mathcal{L}_{M}$ o conjunto de todas as sentenças da linguagem.

Definição 6.1 Um modelo $S_{3}$-Kripke é uma tripla $\mathcal{M}=\left\langle W, R, v_{S}^{3}\right\rangle$.

A semântica de $S_{3}$-Kripke é uma relação $\models_{S}^{3} \subseteq W \times \mathcal{L}_{M}$ dada pelas seguintes regras, em que $w \in W$ e $p^{\circ}$ é um literal:

FWM1. $\mathcal{M}, w \models_{S}^{3} p^{\circ}$ sse $v_{S}^{3}(w)\left(p^{\circ}\right)=1$

FWM2. $\mathcal{M}, w \models_{S}^{3} \phi \wedge \psi$ sse $\mathcal{M}, w \models_{S}^{3} \phi$ e $\mathcal{M}, w \models_{S}^{3} \psi$ 
FWM3. $\mathcal{M}, w \models_{S}^{3} \phi \vee \psi$ sse $\mathcal{M}, w \models_{S}^{3} \phi$ ou $\mathcal{M}, w \models_{S}^{3} \psi$

FWM4. $\mathcal{M}, w \models_{S}^{3} \phi \supset \psi$ sse $\mathcal{M}, w \models_{S}^{3} \phi$ ou $\mathcal{M}, w \models_{S}^{3} \psi$

FWM5. $\mathcal{M}, w \models_{S}^{3} \square \phi$ sse para todo $t \in W$ tal que $w R t, \mathcal{M}, t \models_{S}^{3} \phi$

FWM6. $\mathcal{M}, w \models_{S}^{3} \diamond \phi$ sse existe $t \in W$ tal que $w R t$ e $\mathcal{M}, t \models_{S}^{3} \phi$

FWM7. se $\phi \in S, \mathcal{M}, w \models_{S}^{3} \neg \phi$ sse $\mathcal{M}, w \models_{S}^{3} \phi$

FWM8. não acontece simultaneamente que $\mathcal{M}, w \nvdash_{S}^{3} \phi$ e $\mathcal{M}, w \nvdash_{S}^{3} \neg \phi$

Os conceitos de $S_{3}$-satisfatibilidade, $S_{3}$-validade e $S_{3}$-insatisfatibilidade modais são definidos como usualmente. Vale um análogo ao Teorema 5.1. Antes de enunciá-lo e demonstrá-lo, é necessária uma definição:

Definição 6.2 Um mundo $t \in W$ é $R$-ligado a $w \in W$ sse existe uma sequência finita $\left\{w_{0}, \ldots, w_{n}\right\} \subseteq W$ e tal que $w_{i-1} R w_{i}(1 \leq i \leq n), w_{0}=w$ e $w_{n}=t$.

Observa-se que $w$ é trivialmente $R$-ligado a $w$ (tome uma seqüência com apenas um elemento). Antes de prosseguir, uma notação que simplificará a apresentação. Seja $w^{*}$ o conjunto de todos os mundos $R$-ligados a $w$. Assim, $t \in w^{*}$ sse $t$ for $R$-ligado a $w$.

Segue o

Teorema 6.3 A verdade de uma sentença $\psi$ para um mundo $w$ é determinada por $v_{S}^{3}$ e pelo conjunto

$$
\left\{\neg \phi(t) \text { para todo } t \in w^{*} \text { tal que } \mathcal{M}, t \models_{S}^{3} \phi, \phi \notin S\right\},
$$

É possivel se restringir às sentenças $\neg \phi$ que são subsentenças de $\psi$. A notação $\neg \phi(t)$ significa que a verdade de $\neg \phi$ deve ser avaliada no mundo $t \in W$.

Dem: Para os conectivos $\wedge, \vee, \supset, \square$ e $\diamond$, é imediato que a verdade de $\phi$ é determinada pela das subsentenças para $w$ e para todos os outros $t \in W$ $R$-ligados a $w$. Se $\phi \in S$, então a cláusula FWM7 implica que a verdade (ou falsidade) de $\neg \phi$ em $w$ é equivalente à falsidade (ou verdade) $\phi$ em $w$. Suponha, então, que $\phi \notin S$. Se $\mathcal{M}, w \models_{S}^{3} \phi$, a cláusula FWM8 implica que $\mathcal{M}, w \models_{S}^{3} \neg \phi$. $\mathrm{O}$ mesmo sucede a todos os outros $t \in W R$-ligados a $w$. Restam apenas a função $v_{S}^{3}$ e as sentenças em

$$
\left\{\neg \phi(t) \text { para todo } t \in w^{*} \text { tal que } \mathcal{M}, t \models_{S}^{3} \phi, \phi \notin S\right\}
$$

para determinar a verdade de $\phi$ em $w$.

Observa-se que a função $v_{S}^{3}$ precisa ser avaliada apenas nos mundos que estão $R$-ligados a $w$ para determinar a verdade de qualquer sentença $\psi$ em $w$. O conjunto $\left\{\neg \phi(t)\right.$ para todo $t \in w^{*}$ tal que $\left.\mathcal{M}, t \models_{S}^{3} \phi, \phi \notin S\right\}$ é formado pelas negações de todas as sentenças cujos átomos não estão todos no conjunto das proposições relevantes e que são verdadeiras em $w$ e nos outros mundos $R$-ligados a $w$. Nessas sentenças há liberdade, pela satisfação das condições FMW7 e 
FWM8, de determinar a verdade de suas negações. Além disso, a verdade de todas essas sentenças influencia a verdade de qualquer sentença $\phi$ em $w$.

$O$ conceito de tradução de uma sentença da Seção 5.1 pode ser estendido. Só que, aqui, a tradução depende do mundo $t \in W$ em que se está avaliando a verdade. Assim, seja $p_{\neg \phi, t}$ um novo átomo (para cada $\phi \notin S$ tal que $\mathcal{M}, t \nvdash_{S}^{3} \phi$ para todo $\left.t \in w^{*}\right)$ cuja valoração é verdadeira se $\mathcal{M}, t \models_{S}^{3} \neg \phi$ e falsa caso contrário, e defina a tradução $\phi^{\bullet S, t}$ de $\phi$ da seguinte maneira:

$$
\begin{aligned}
p^{\bullet S, t} & =p \\
(\phi \wedge \psi)^{\bullet S, t} & =\phi^{\bullet S, t} \wedge \psi^{\bullet S, t} \\
(\phi \vee \psi)^{\bullet S, t} & =\phi^{\bullet S, t} \vee \psi^{\bullet S, t} \\
(\phi \supset \psi)^{\bullet S, t} & =\phi^{\bullet S, t} \supset \psi^{\bullet S, t} \\
(\square \phi)^{\bullet S, t} & =\square\left(\phi^{\bullet S, t}\right) \\
(\diamond \phi)^{\bullet S, t} & =\diamond\left(\phi^{\bullet S, t}\right) \\
(\neg \phi)^{\bullet S, t} & = \begin{cases}\neg\left(\phi^{\bullet S, t}\right) & \text { se } \phi \in S \text { ou se } \mathcal{M}, t \models_{S}^{3} \phi \\
p_{\neg \phi, t} & \text { se } \phi \notin S \text { e } \mathcal{M}, t \models_{S}^{3} \phi\end{cases}
\end{aligned}
$$

Como no caso proposicional, a partir do conceito de tradução seguem alguns teoremas.

Teorema 6.4 Uma sentença $\phi$ é $S_{3}$-satisfativel em algum mundo $w$ de um modelo $\mathcal{M}$ sse $\phi^{\bullet S, w}$ é satisfativel classicamente para $w$ em $\mathcal{M}$.

Dem: A tradução mantém inalteradas as porções não clássicas da semântica. Assim, os átomos são traduzidos em si próprios, e a tradução da necessitação, possibilitação, disjunção, conjunção e implicação é a necessitação, possibilitação, disjunção, conjunção e implicação da tradução de seus componentes. Isso reflete o fato de que a semântica não altera as propriedades desses conectivos. Por outro lado, quando $\phi \notin S$ e $\mathcal{M}, t \models_{S}^{3} \phi$ para algum $t R$-ligado a $w-$ e apenas quando isso acontece - , há liberdade de escolha para a verdade de $\neg \phi$ em $t$. A tradução toma conta disso considerando $\neg \phi$ como um novo átomo $p_{\neg \phi, t}$ (informalmente, a tradução "tira" o caráter não clássico da semântica). Se a sentença como um todo for satisfatível pelo mundo e modelo sob a tradução, ela $S_{3}$-satisfatível nos mesmos mundos e modelo, e vice-versa.

Teorema 6.5 Seja Prop $(\phi)$ o conjunto dos átomos de $\phi$. Toda sentença $\phi$ com $\operatorname{Prop}(\phi) \cap S=\emptyset$ é $S_{3}$-satisfativel.

Dem: Seja $w$ um mundo de um modelo qualquer. Como $\operatorname{Prop}(\phi) \cap S=\emptyset$, a tradução $(\phi)^{\bullet S, w}$ leva a um fragmento sem negação da lógica clássica, e toda sentença dessa forma é satisfatível em qualquer modelo (basta valorar todos os átomos como verdadeiros em todos os mundos). Pelo Teorema 6.4, a sentença $\phi$ é $S_{3}$-satisfatível.

Monotonicidade e convergência também valem.

Teorema 6.6 (Monotonicidade) Seja $S \subseteq S^{\prime} \subseteq A$. Se uma sentença $\phi e ́$ $S_{3}$-válida, então é também $S_{3}^{\prime}$-válida. 
Dem: A demonstração é por indução sobre as subsentenças. Seja $\mathcal{M}$ um modelo e $w$ um mundo desse modelo. Se $\phi$ é $S_{3}$-válida, então, para esse modelo em particular, ocorre $\mathcal{M}, w \models_{S}^{3} \phi$. As condições FWM1-6 e FWM8 não dependem de $S$ e, portanto, de $S^{\prime}$. Assim, somente a condição FWM7 precisa ser analisada. Suponha que alguma subsentença de $\phi$ seja da forma $\neg \psi$, e que $\mathcal{M}, t \models_{S}^{3} \neg \psi$ para algum $t R$-ligado a $w$. Há dois casos em que FWM7 pode ser usada. No primeiro caso, $\psi \in S$; então $\mathcal{M}, t \models_{S}^{3} \neg \psi$ sse $\mathcal{M}, t \models_{S}^{3} \psi$. Como $S \subset S^{\prime}$, então $\psi \in S^{\prime} \supseteq S$. Pela hipótese de indução, $\mathcal{M}, t \not \models_{S^{\prime}}^{3} \psi$. Isso, junto com $\psi \in S^{\prime}$, implica que $\mathcal{M}, t \models_{S^{\prime}}^{3} \neg \psi$. No segundo caso, $\psi \notin S$ e $\psi \in S^{\prime}$. Sabese que $\mathcal{M}, t \models_{S}^{3} \neg \psi$. Se ocorresse $\mathcal{M}, t \models_{S^{\prime}}^{3} \psi$, então pela hipótese de indução necessariamente $\mathcal{M}, t \models_{S}^{3} \psi$. Mas se isso fosse verdade, não seria necessário que $\mathcal{M}, t \models_{S}^{3} \neg \psi$ (haveria liberdade de escolha para a verdade de $\neg \psi$ ), o que não é o caso. Portanto $\mathcal{M}, t \nvdash_{S^{\prime}}^{3} \psi$, o que implica, por FWM7, que $\mathcal{M}, t \models_{S^{\prime}}^{3} \neg \psi$.

Assim, se for tomado $S^{\prime}=A$, conclui-se que se uma sentença é $S_{3}$-válida, então é válida classicamente.

Como anteriormente, vale

Teorema 6.7 (Convergência) Se uma sentença $\phi$ é válida classicamente, então existe $S \subseteq A$ tal que $\phi$ é também $S_{3}$-válida.

Para a prova, considere $S=A$.

Abaixo, alguns exemplos.

1. A Regra da Necessitação vale em uma aproximação $S_{3}$-Kripke: deve-se mostrar que, se $\models_{S}^{3} \phi$, então $\models_{S}^{3} \square \phi$. De fato, fixe um modelo $\mathcal{M}$ e um mundo $w \in W$. Como $\models_{S}^{3} \phi$, em particular para todos os mundos $t \in W$ tais que $w R t$ tem-se que $\mathcal{M}, t \models_{S}^{3} \phi$. Pela cláusula FWM2, $\mathcal{M}, w \models_{S}^{3} \square \phi$.

2. Vale o princípio $K: \models_{S}^{3} \square(p \supset q) \supset(\square p \supset \square q)$. De fato, suponha que $\mathcal{M}, w \models_{S}^{3} \square(p \supset q)$ e $\mathcal{M}, w \models_{S}^{3} \square p$, para um modelo $\mathcal{M}=\left\langle W, R, v_{S}^{3}\right\rangle$ e um mundo $w \in W$ arbitrários. Então, para todo $t \in W$ tal que $w R t$ tem-se $\mathcal{M}, t \models_{S}^{3} p \supset q$ e $\mathcal{M}, t \models_{S}^{3} p$. Assim, para todo $t \in W$ tal que $w R t$, ocorre $\mathcal{M}, t \models_{S}^{3} q$, ou seja, $\mathcal{M}, w \models_{S}^{3} \square q$.

3. Não vale a equivalência $\square \phi \equiv \neg \diamond \neg \phi$. De fato, suponha que $W=\{w\}$, que $R=\{(w, w)\}$, que $v_{S}^{3}(w)(p)=0$ ( $p$ é um átomo) e que $S=\emptyset$. Então, tem-se que $\mathcal{M}, w \nvdash_{S}^{3} p$, logo que $\mathcal{M}, w \nvdash_{S}^{3} \square p$; além disso, $\mathcal{M}, w \models_{S}^{3} \neg p$ e, portanto, $\mathcal{M}, w \models_{S}^{3} \diamond \neg p$. Como $\diamond \neg p \notin S$ e $\mathcal{M}, w \models_{S}^{3} \diamond \neg p$, as condições do Teorema 6.3 são satisfeitas e pode-se assumir que $\mathcal{M}, w \models_{S}^{3} \neg \diamond \neg p$. Alterações simples desse modelo mostram que não vale, de fato, nenhuma das outras equivalências $\neg \square \phi \equiv \diamond \neg \phi, \neg \square \neg \phi \equiv \diamond \phi$.

4. Não vale a equivalência $\square p \supset \square q \equiv \neg \square p \vee \square q$. De fato, suponha que $W=\{w\}$, que $R=\{(w, w)\}$, que $v_{S}^{3}(w)(p)=1$ e $v_{S}^{3}(w)(q)=0(p$ e $q$ são átomos) e que $S=\emptyset$. Então, tem-se que $\mathcal{M}, w \models_{S}^{3} p$ e portanto que $\mathcal{M}, w \models_{S}^{3} \square p$. Como $\mathcal{M}, w \nvdash_{S}^{3} q$, tem-se que $\mathcal{M}, w \nvdash_{S}^{3} \square q$. Logo, $\mathcal{M}, w \nvdash_{S}^{3} \square p \supset \square q$. Como $\square p \notin S$ e $\mathcal{M}, w \models_{S}^{3} \square p$, as condições do Teorema 6.3 são satisfeitas e pode-se assumir que $\mathcal{M}, w \models_{S}^{3} \neg \square p$, implicando que $\mathcal{M}, w \models_{S}^{3} \neg \square p \vee \square q$. 
Uma fonte de complexidade nas sentenças modais é a interação de operadores modais. Como controlar essa interação? Uma possível solução é modificar FWM1-8 com a adição de um novo parâmetro, o chamado limite máximo de introspecção. Esse parâmetro toma valores no conjunto dos números inteiros, positivos, negativos ou zero. Intuitivamente, esse limite determina o quanto um agente pode raciocinar sobre suas crenças das suas crenças ... das suas crenças. ${ }^{1}$ A semântica, a ser chamada semântica modal $\left\langle S_{3}, n\right\rangle$-Kripke - e que dá origem aos modelos $\left\langle S_{3}, n\right\rangle$-Kripke, é dada pelas regras abaixo, em que $n$ é o limite máximo de introspecção:

FWD1. $\mathcal{M}, w \models_{S, n}^{3} p^{\circ}$ sse $v_{S}^{3}(w)\left(p^{\circ}\right)=1$

FWD2. $\mathcal{M}, w \models_{S, n}^{3} \phi \wedge \psi$ sse $\mathcal{M}, w \models_{S, n}^{3} \phi$ e $\mathcal{M}, w \models_{S, n}^{3} \psi$

FWD3. $\mathcal{M}, w \models_{S, n}^{3} \phi \vee \psi$ sse $\mathcal{M}, w \models_{S, n}^{3} \phi$ ou $\mathcal{M}, w \models_{S, n}^{3} \psi$

FWD4. $\mathcal{M}, w \models_{S, n}^{3} \phi \supset \psi$ sse $\mathcal{M}, w \models_{S, n}^{3} \phi$ ou $\mathcal{M}, w \models_{S, n}^{3} \psi$

FWD5. $\mathcal{M}, w \models_{S, n}^{3} \square \phi$ sse $n>0$ e para todo $t \in W$ tal que $w R t$, $\mathcal{M}, t \models_{S, n-1}^{3} \phi$

FWD6. $\mathcal{M}, w \models_{S, n}^{3} \diamond \phi$ sse ou $n \leq 0$ ou existe $t \in W$ tal que $w R t$ e $\mathcal{M}, t \models \models_{S, n-1}^{3} \phi$

FWD7. se $\phi \in S, \mathcal{M}, w \models_{S, n}^{3} \neg \phi$ sse $\mathcal{M}, w \nvdash_{S, n}^{3} \phi$

FWD8. não acontece simultaneamente que $\mathcal{M}, w \nvdash_{S, n}^{3} \phi$ e $\mathcal{M}, w \nvdash_{S, n}^{3} \neg \phi$

Essa semântica introduz um novo parâmetro para a determinação da verdade de sentenças em um modelo; além da verdade depender do mundo e do conjunto de proposições relevantes $S$, ela depende de um número $n$ que, intuitivamente, dá a capacidade máxima de introspeç̧ão do agente. Fora desse limite (ou seja, quando $n \leq 0$ ), nada é necessário e tudo é possível, como o mostram as condições FWD5-6. Os conceitos de $\left\langle S_{3}, n\right\rangle$-satisfatibilidade, $\left\langle S_{3}, n\right\rangle$-validade e $\left\langle S_{3}, n\right\rangle$ insatisfatibilidade modais são definidos como usualmente. Observa-se apenas que uma sentença $\phi$ é válida quando, para todo modelo $\mathcal{M}$ e mundo $w$ desse modelo, ocorrer $\mathcal{M}, w \models_{S, n}^{3} \phi$ : o $n$ é fixo, ou seja, não se solicita que $\mathcal{M}, w \models_{S, n}^{3}$ $\phi$ para todo $n$.

Como a semântica da negação permanece igual, o Teorema 6.3 continua valendo (basta substituir, no enunciado e demonstrações, $\models_{S}^{3}$ por $\models_{S, n}^{3}$ para $n$ adequados). A tradução de uma sentença $\phi$ seria essencialmente idêntica à tradução na semântica dada pelas regras FWM1-8, com a diferença que, para $n \leq 0$, toda necessitação é falsa e toda possibilitação é verdadeira. O Teorema 6.4 é, portanto, válido e pelas mesmas razões. O Teorema 6.5 também é válido,

\footnotetext{
${ }^{1}$ Quando $n=0$, a intuição é a de o agente pensante que não raciocina sobre nenhuma crença; quando $n<0$, a intuição é a de que o agente não consegue nem raciocinar sobre os próprios fatos do mundo em que está, e não conseguiria fazê-lo a menos que tivesse um poder de introspeção $|n|$ passos maior.
} 
levando em conta que toda necessitação é levada, pela tradução, a uma sentença inválida quando $n \leq 0$ e que, em alguns casos, isso dá origem a sentenças inválidas classicamente (e portanto $\left\langle S_{3}, n\right\rangle$-inválidas). Por fim, monotonicidade e convergência são válidos pelas mesmas razões de antes; a única coisa que acontece, quando se limita a capacidade máxima de introspecção do agente, é que a verdade de um menor número de subsentenças precisa ser verificada.

Para os exemplos a seguir, supõe-se que $S=A$, ou seja, que o conjunto de proposições relevantes é o conjunto de todos os átomos. Com isso, a negação se comporta classicamente e apenas os operadores $\square$ e $\diamond$ mostram comportamentos diferentes.

1. Necessitação não vale em uma aproximação $\left\langle S_{3}, n\right\rangle$-Kripke. Seja $\mathcal{M}$ um modelo qualquer e $w$ um mundo desse modelo e suponha que $n=0$. Então, seja qual for a sentença $\phi$ (e que pode ser uma tautologia, sempre satisfeita por qualquer modelo), nunca ocorre $\mathcal{M}, w \models_{S, 0}^{3} \square \phi$, porque a condição FMD5 exige, em primeiro lugar, que $n>0$ para que uma sentença do tipo $\square \phi$ seja considerada verdadeira. Portanto, essa lógica não é normal. Observa-se que esse exemplo se aplica a todo $n \leq 0$ e a nenhum $n>0$.

2. Vale o princípio $K: \models_{S, n}^{3} \square(p \supset q) \supset(\square p \supset \square q)$. Há dois casos a considerar. Primeiro, suponha que $n>0$. Então, se em um modelo $\mathcal{M}=$ $\left\langle W, R, v_{S}^{3}\right\rangle$ e em um mundo $w \in W$ desse modelo ocorrerem $\mathcal{M}, w \models_{S, n}^{3}$ $\square(p \supset q)$ e $\mathcal{M}, w \models_{S, n}^{3} \square p$, tem-se, por FWD5, que para todo $t \in W$ tal que $w R t$ ocorre $\mathcal{M}, t \models_{S, n}^{3} p \supset q$ e $\mathcal{M}, t \models_{S, n}^{3} p$. Isso, por FWD4, implica que $\mathcal{M}, t \models_{S, n}^{3} q$ para todo $t \in W$ tal que $w R t$, ou seja, $\mathcal{M}, w \models_{S, n}^{3} \square q$. No segundo caso, $n \leq 0$. Pela cláusula FWD5, seja qual for o modelo $\mathcal{M}$ e um mundo $w$ desse modelo, $\mathcal{M}, w \forall_{S, n}^{3} \square(p \supset q)$. Portanto, por FWD4 (como o antecedente de $\square(p \supset q) \supset(\square p \supset \square q$ ) é sempre falso), tem-se que $\mathcal{M}, w \models_{S, n}^{3} \square(p \supset q) \supset(\square p \supset \square q)$.

3. Vale a equivalência $\diamond \phi \equiv \neg \square \neg \phi$. De fato, seja $\mathcal{M}$ um modelo, $w$ um mundo desse modelo, e suponha que $\mathcal{M}, w \models_{S, n}^{3} \diamond \phi$. Pela condição de verdade FWD6, ou $n \leq 0$ ou existe $t$ tal que $w R t$ e $\mathcal{M}, t \models_{S, n-1}^{3} \phi$. Assim, não é verdade que $n>0 \mathrm{e}$, para todo $t$ tal que $w R t$, não ocorra $\mathcal{M}, t \models_{S, n-1}^{3} \phi$ (ou seja, como a negação se comporta classicamente, ocorra $\left.\mathcal{M}, t \models_{S, n-1}^{3} \neg \phi\right)$. Pela condição de verdade FWD5, isso é o mesmo que dizer que não é verdade que $\mathcal{M}, w \models_{S, n}^{3} \square \neg \phi$. Assim, $\mathcal{M}, w \models_{S, n}^{3} \neg \square \neg \phi$. Similarmente, valem todas as outras equivalências $\neg \square \phi \equiv \diamond \neg \phi, \neg \square \neg \phi \equiv$ $\diamond \phi$.

Os exemplos 1 e 3 mostram que, de uma certa forma, o controle de introspeç̧ão realiza efeitos ortogonais (ou seja, independentes) dos efeitos realizados pela seleção de um conjunto de proposições relevantes: compare com os exemplos 1 e 3 dados em conexão com a semântica FWM1-8. 


\subsection{Tableaux de Prova}

As regras para SST têm a propriedade da modularidade. Apenas algumas regras são válidas para todas as lógicas modais básicas. Uma teoria de prova para lógicas modais que seja o mais geral possível não deveria, em princípio, mexer em propriedades específicas de uma determinada lógica, mas apenas nas propriedades que valem para todas. As regras SST são dadas pelas Tabelas 2.8, 2.9 e 2.10 , mas apenas as duas primeiras tratam de regras que valem para todas as lógicas.

O comportamento não clássico do operador $\neg$ fora do conjunto de proposições relevantes é descrito por uma porção da regra neg modificada

$$
\frac{\sigma: T \neg \phi}{\sigma: F \phi} \text { desde que } \phi \in S
$$

Observa-se que, se $\phi \notin S$, a regra não pode ser aplicada. Essa é a única alteração nas regras da Tabela 2.8. A regra será chamada de $\left(T \neg^{\prime}\right)$. A outra regra neg, que permite inferir $T \phi$ de $F \neg \phi$, é mantida inalterada, podendo ser referida como regra $(F \neg)$.

O controle de introspecção, dado pelo limite $n$, é incorporado na seguinte modificação da regra $(\pi)$, a única que permite a introdução de novos prefixos. Essa regra, que será chamada de $\left(\pi^{\prime}\right)$, é dada por

$$
\frac{\sigma: \pi}{\sigma . m: \pi_{0}} \text { desde que } \sigma . m \text { seja novo no ramo e }|\sigma|<n
$$

Observa-se que, se $|\sigma|>n$, a regra não pode ser aplicada. Essa é a única alteração nas regras da Tabela 2.9.

Além disso, é necessária uma nova regra de fechamento dos tableaux, para incorporar neles aquilo que já foi observado na Seção 6.1, isto é, que além do limite de introspecção nada é necessário e tudo é possível. Nos tableaux considerados até ägora, um ramo fechava somente quando nele apareciam uma sentença e sua conjugada. A nova regra é a seguinte:

(bey) Um ramo $R$ do tableau fecha sempre que nele aparece uma sentença da forma $\sigma: \nu$, quando $|\sigma| \geq n$, ou seja, quando o comprimento do prefixo fica no ou além do limite máximo de introspecção $n$

Essa regra, chamada de (bey) (de "beyond", "além") será melhor explicada após a definição de satisfatibilidade dos ramos de um tableau (Definição 6.10). Ela é a única regra não-clássica de fechamento.

Definição 6.8 Tableaux obtidos utilizando as regras $\left(T \neg^{\prime}\right) e\left(\pi^{\prime}\right)$ e as outras regras SST sem alteração, com a regra de fechamento clássica mais a regra de fechamento (bey), são chamados de $\left\langle S_{3}, n\right\rangle$-SST $\left(\left\langle S_{3}, n\right\rangle\right.$-tableaux passo a passo).

A seguir apresentam-se exemplos de aplicação dos $\left\langle S_{3}, n\right\rangle$-SST. Provas que utilizarem a regra de fechamento (bey) são não-clássicas, já que essa é uma regra não-clássica. 


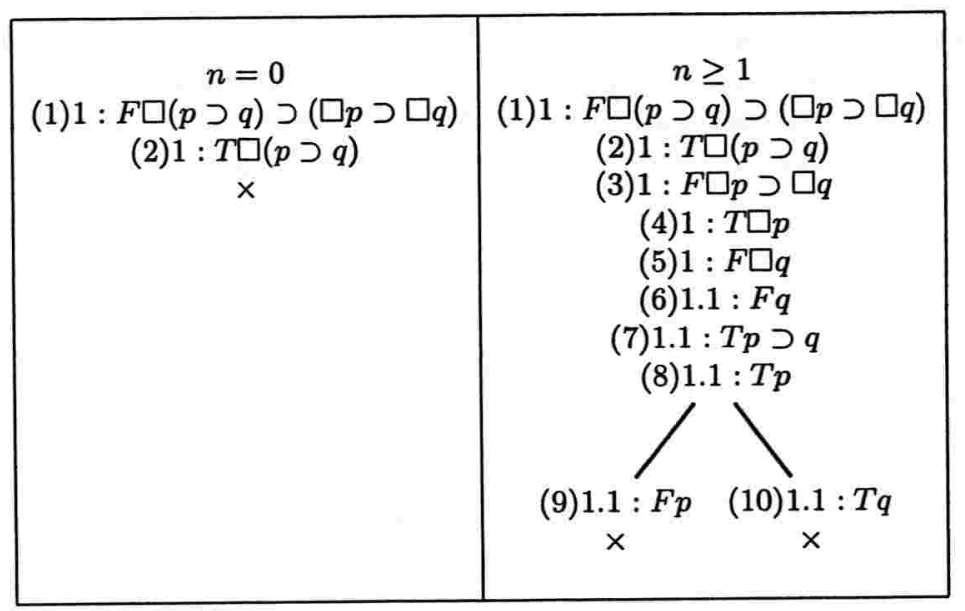

Figura 6.1: Prova de $K$ por $\left\langle S_{3}, n\right\rangle$-SST

Na Figura 6.1 há uma prova de $K$. No lado esquerdo, fixou-se o limite máximo de introspecção $n=0$ e, no lado direito, $n \geq 1$. A sentença (2) do lado esquerdo foi obtida de (1) pela regra $\alpha$; pela regra de fechamento (bey), como $0=|1| \geq 0^{2}$, o tableau fecha. Do lado direito, as sentenças (2)-(3) são obtidas de (1) pela regra $\alpha$. As sentenças (4)-(5) são obtidas de (3) por $\alpha$ também. A sentença (6) é obtida de (5) por aplicação da regra $\left(\pi^{\prime}\right)$, uma vez que $0=|1|<1$. As sentenças (7) e (8) são obtidas de (2) e (4), respectivamente, por aplicação da regra $(K)$. Por fim, as sentenças (9)-(10) são obtidas de (7) por $\beta$. Importante observar, nesta prova, que embora o princípio $K$ seja sempre verdadeiro (como se pode obter a partir da semântica) as demonstrações são distintas para $n=0$ e $n \geq 1$.

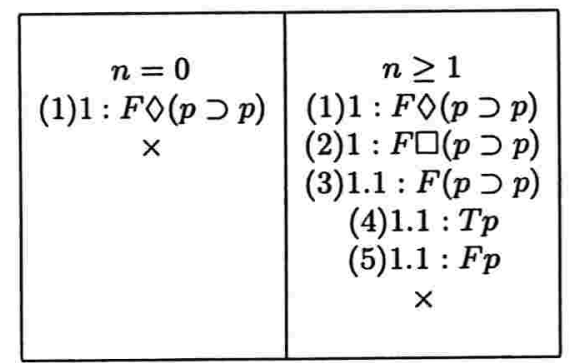

Figura 6.2: Prova de $\diamond(p \supset p)$ na lógica modal D por $\left\langle S_{3}, n\right\rangle$-SST

Na Figura 6.2 há uma prova de $\diamond(p \supset p)$ na lógica modal D. Essa lógica possui, em adição aos princípios válidos para todas as lógicas (regras proposicionais, $(L o c),(G l o b)$ e $\left.\left(\pi^{\prime}\right)\right)$, as regras $(K)$ e $(D)$. No lado esquerdo, fixou-se o limite máximo de introspecção $n=0 \mathrm{e}$, no lado direito, $n \geq 1$. No lado

${ }^{2}$ Lembrar a definição do comprimento de prefixos, dada na Seção 2.2.2. 
esquerdo, a regra de fechamento (bey) é imediatamente aplicável, resultando no fechamento do tableau. No lado direito, a sentença (2) é obtida de (1) pela regra $(D)$. A sentença (3) é obtida de $(2)$ por $\left(\pi^{\prime}\right)$, porque $0=|1|<1$. As sentenças (4) e (5) são obtidas de (3) por aplicação da regra $\alpha$.

\begin{tabular}{|c|c|}
\hline & $n \geq 0$ \\
$(1) 1: F \diamond(p \supset \square p)$ & $(1) 1: F \diamond(p \supset \square p)$ \\
$\times$ & $(2) 1: F(p \supset \square p)$ \\
& $(3) 1: T p$ \\
& $(4) 1: F \square p$ \\
& $(5) 1.1: F p$ \\
& $(6) 1.1: F(p \supset \square p)$ \\
& $(7) 1.1: T p$ \\
& $\times$ \\
\hline
\end{tabular}

Figura 6.3: Prova de $\diamond(p \supset \square p)$ na lógica modal $\mathbf{T}$ por $\left\langle S_{3}, n\right\rangle$-SST

Na Figura 6.3 há uma prova de $\diamond(p \supset \square p)$ na lógica modal T. Essa lógica possui, em adição aos princípios válidos para todas as lógicas, as regras $(K)$ e $(T)$. No lado esquerdo, fixou-se o limite máximo de introspeç̧ão $n=0$ e, no lado direito, $n \geq 1$. No lado esquerdo, como no caso anterior, a regra (bey) é imediatamente aplicável, fechando o tableau. A sentença (2), no lado direito, é obtida é obtida de (1) pela regra $(T)$. As sentenças (3) e (4) são obtidas de (2) por aplicação da regra $\alpha$. A sentença (5) é obtida de (4) pela regra $\left(\pi^{\prime}\right)$. A sentença (6) é obtida de (1) por aplicação da regra $(K)$, e (7) é obtida de (6) por aplicação da regra $\alpha$.

$$
\begin{array}{|c|c|c|}
\hline & & \\
(1) 1: F \square p \supset \square \square \square p & (1) 1: F \square p \supset \square \square \square p & n \geq 2 \\
(2) 1: T \square p & (2) 1: T \square p & (2) 1: T \square p \square \square \square p \\
\times & (3) 1: F \square \square \square & (3) 1: F \square \square \square p \\
& (4) 1: T p & (4) 1: T p \\
& (5) 1.1: F \square \square p & (5) 1.1: F \square \square p \\
& (6) 1.1: T \square p & (6) 1.1: T \square p \\
& \times & (7) 1.1 .1: F \square p \\
& & (8) 1.1 .1: T \square p \\
& & \times \\
\hline
\end{array}
$$

Figura 6.4: Prova de $\square p \supset \square \square \square p$ na lógica modal S5 por $\left\langle S_{3}, n\right\rangle$-SST

Na Figura 6.4 há uma prova de $\square p \supset \square \square \square p$ na lógica modal S5. Essa lógica possui, em adição aos princípios válidos para todas as lógicas, as regras $(K)$, $(T),(4)$ e $\left(4^{R}\right)$. No lado esquerdo, fixou-se o limite máximo de introspecção $n=0$, no meio fixou-se $n=0$ e, no lado direito, $n \geq 1$. No lado esquerdo, a 
sentença (2) é obtida de (1) por $\alpha$; isso fecha o tableau, por (bey). No meio e no lado esquerdo, as sentenças (2)-(3) são obtidas de (1) pela regra $\alpha$. A sentença (4), nessas duas colunas, é obtida de (2) por aplicação da regra $(T)$. Aplicando a regra $\left(\pi^{\prime}\right)$ à sentença (3), obtém-se a sentença (5) nessas duas colunas. A sentença (6) (no meio e no lado direito) é obtida de (2) por aplicação da regra (4). Como $1=|1.1| \geq 1$, a sentença (6) na coluna do meio fecha o tableau, por (bey). No lado direito, com a regra $\left(\pi^{\prime}\right)$ obtém-se (7) a partir de (5). Por fim, a sentença (8) é obtida de (6) pela regra (4). O tableau nesse lado fecha, seja pelo aparecimento de uma contradição (sentenças (7) e (8)), seja pelo aparecimento da sentença (8), para a qual a regra (bey) é aplicável, já que $2=|1.1 .1| \geq 2$. Esse exemplo mostra duas coisas. Primeiro, um tableau pode fechar simultaneamente por causa das duas regras de fechamento; nesses casos, assume-se que o tableau fechou por causa da regra clássica - isso porque é uma aproximação da demonstração clássica que se busca. Segundo, quanto maior o número de interações modais nas sentenças, maior deve ser o limite máximo de introspecção para que se possa demonstrá-las classicamente (sem utilizar (bey)).

Todos os exemplos apresentados até agora mostram que, quando o limite máximo de introspecção é suficientemente pequeno e a regra (bey) é aplicável, o tamanho das demonstrações pode diminuir consideravelmente. No último exemplo, dado abaixo, a regra (bey) não pode ser aplicada, com o resultado de que, se o limite máximo de introspeç̧ão não for aumentado, o ramo do tableau não fecha, ficando "bloqueado" (o que é representado com um ponto de interrogação, ?, abaixo do último nó do tableau). Esse exemplo também combina as duas aproximações, a proposicional, que seleciona um subconjunto $S$ de proposições relevantes, e a modal, que determina um limite máximo de introspecção $n$.

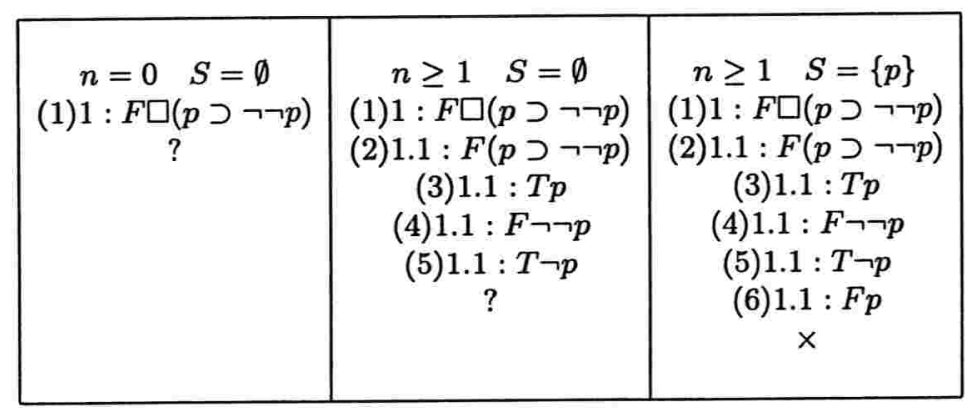

Figura 6.5: Prova de $\square(p \supset \neg \neg p)$ por $\left\langle S_{3}, n\right\rangle$-SST

A Figura 6.5 dá uma prova de $\square(p \supset \neg \neg p)$. No lado esquerdo, fixou-se o limite máximo de introspecção $n=0$ e tomou-se $S=\emptyset$; no meio, supôs-se que $n \geq 1$ mas ainda mantém-se $S=\emptyset$; no lado direito, $n \geq 1$ e adiciona-se o átomo $p$ a $S$. No lado esquerdo, nada pode ser feito, já que é preciso aplicar a regra $\left(\pi^{\prime}\right)$ para gerar um prefixo com comprimento igual a 1 , e quando $n=0$ a regra não pode ser aplicada. No meio e no lado direito, como essa condição pode ser 
satisfeita, prossegue-se a demonstração. A sentenças (3)-(4) (no meio e no lado direito) são obtidas de (2) por aplicação da regra $\alpha$, e (5) é obtida de (4) por aplicação da regra $(F \neg)$. No meio, não há mais como prosseguir, pois $p \notin S$. À direita, obtém-se (6) de (5) a partir da regra $\left(T \neg^{\prime}\right)$. Nesse exemplo é visto como o controle sobre a demonstração é fino.

Na Figura 6.5, os tableaux abertos na esquerda e no meio permitem construir um contra-modelo à sentença que se desejava provar. No lado esquerdo, como $n=0$, qualquer modelo $\mathcal{M}$ satisfaz $1: F \square(p \supset \neg \neg p)$. De fato, isso significa que $\mathcal{M}, 1 \not \models_{S, 0}^{3} \square(p \supset \neg \neg p)$ o que, por FWD5, é sempre verdade. No meio, como $S=\emptyset$ (e, evidentemente, $p \notin S$ ), pode-se assumir $\mathcal{M}, 1 \models_{S, 0}^{3} p$ e $\mathcal{M}, 1 \models_{S, 0}^{3} \neg p$. Dessa forma, $\mathcal{M}, 1 \nvdash_{S, 0}^{3} \neg \neg p$, que implica que $\mathcal{M}, 1 \nvdash_{S, 0}^{3} p \supset \neg \neg p$, e assim, que $\mathcal{M}, 1 \not \models_{S, 1}^{3} \square(p \supset \neg \neg p)$ (por FWD5).

Há que se provar correção e completude dos $\left\langle S_{3}, n\right\rangle$-SST com relação à semântica FWD1-8 dada na seção anterior.

Lembra-se aqui que, para provar a correção de um tableau com relação a determinada semântica, basta provar que, se um tableau é satisfatível por determinado modelo e sobre esse tableau aplica-se determinada regra, então o tableau estendido continua sendo satisfatível pelo modelo. Os prefixos intuitivamente designam mundos, e o promeiro passo da demonstração da correção é formalizar isso.

Definição 6.9 Seja $R$ um ramo do tableau e $\mathcal{M}=\left\langle W, R, v_{S}^{3}\right\rangle$ um modelo $\left\langle S_{3}, n\right\rangle$-Kripke. Uma interpretação SST é uma função $i$ dos prefixos que ocorrem em $R$ para mundos $i(\sigma) \in W$ tal que, para todo $\sigma$ e $\sigma . n$ presentes em $R$, tenha-se $i(\sigma) R i(\sigma . n)$.

O requisito $i(\sigma) R i(\sigma . n)$ formaliza o fato de que o mundo designado pelo prefixo $\sigma . n$ sempre deve ser acessível ao mundo designado pelo prefixo $\sigma$. delo.

A seguir, define-se o conceito de um ramo satisfativel por determinado mo-

Definição 6.10 Um ramo do tableau $R$ com hipóteses locais $\Gamma_{U}$ e globais $\Gamma_{G}$ é satisfatível sse existe um modelo $\mathcal{M}=\left\langle W, R, v_{S}^{3}\right\rangle$ e uma interpretação $S S T i$ tais que:

- $\mathcal{M}$ valida $\Gamma_{G}$ (ou seja, cada sentença de $\Gamma_{G}$ )

- o mundo $i(1)$ satisfaz $\Gamma_{U}$ (ou seja, cada sentença de $\Gamma_{U}$ ) em $\mathcal{M}$

- para cada sentença prefixada $\sigma$ : Th em $R$, tem-se $\mathcal{M}, i(\sigma) \models_{S, n-|\sigma|}^{3} \phi$, e para cada sentença prefixada $\sigma: F \phi$ em $R$, tem-se $\mathcal{M}, i(\sigma) \nvdash_{S, n-|\sigma|}^{3} \phi$

Um tableau é satisfativel se pelo menos um de seus ramos o for.

Com essas noções, é possível justificar a razão de existência da regra (bey). Suponha que no tableau apareça uma sentença $\sigma: \nu$, sendo que $|\sigma| \geq n$. Então $n-|\sigma| \leq 0$ Considere o caso em que $\nu=T \square \phi$. Se essa sentença estivesse em um ramo satisfatível $R$, existiria em algum modelo $\mathcal{M}$ e uma interpretação SST $i$ tais que $\mathcal{M}, i(\sigma) \models_{S, n-|\sigma|}^{3} \square \phi$. Mas, por FWD5, isso é impossível. Da mesma 
forma, por FWD6 é impossível que um ramo do tableau seja satisfatível com uma sentença $\sigma: F \diamond \phi$. Assim, a existência de uma sentença $\sigma: \nu$ em que $|\sigma| \geq n$ em um ramo implica no fechamento do mesmo.

A regra (bey) vem a ser, em essência, equivalente a introduzir uma outra regra de expansão da seguinte forma:

$$
\sigma: \pi \text { desde que }|\sigma| \geq n
$$

Isso permite introduzir sempre uma sentença da forma $\sigma: \pi$ para qualquer prefixo $\sigma$ com comprimento maior ou igual ao limite máximo de introspeç̧ão. $\mathrm{Ou}$ seja, é uma regra sem premissas. Assim que aparecer no tableau uma sentença $\sigma: \nu$ para $|\sigma| \geq n$, a introdução de $\sigma: \pi$, por essa regra de expansão, faz surgir um par de sentenças conjugadas, o que permite fechar o tableau. Como essa regra de expansão serviria, na prática, apenas para fechar os tableaux, preferiu-se neste texto trabalhar com uma nova regra de fechamento.

Teorema 6.11 (Correção) Suponha que um $\left\langle S_{3}, n\right\rangle-S S T$ para $\Gamma_{U} \cup \Gamma_{G} \vdash \psi$ feche, em que $\Gamma_{U}$ é o conjunto de hipóteses locais e $\Gamma_{G}$ o conjunto de hipóteses globais. Então $\Gamma_{G} \models_{S, n}^{3} \Gamma_{U} \Rightarrow \psi$.

Dem: Como observado acima, é suficiente mostrar que se um tableau é satisfatível, ele continua satisfatível sob a aplicação das regras SST. Isso será feito apenas para as regras não clássicas $\left(T \neg^{\prime}\right)$ e $\left(\pi^{\prime}\right)$; os outros casos são iguais ao caso clássico (pois não há alteração das outras regras) e podem ser encontrados em [Mas00].

Suponha, portanto, que o ramo $R$ de um tableau satisfatível possua um nó da forma $\sigma: T \neg \phi$. Seja $\mathcal{M}$ o modelo e $i$ a interpretação SST que satisfazem esse ramo. Então $\mathcal{M}, i(\sigma) \models_{S, n-|\sigma|}^{3} \neg \phi$. Suponha que $\phi \in S$, de forma que a regra $\left(T \neg^{\prime}\right)$ possa ser aplicada. Nesse caso, por FWD7, tem-se $\mathcal{M}, i(\sigma) \models_{S, n-|\sigma|}^{3} \neg \phi$ sse $\mathcal{M}, i(\sigma) \nvdash_{S, n-|\sigma|}^{3} \phi$. Mas a aplicação da regra $\left(T \neg^{\prime}\right)$ leva a um novo nó da forma $\sigma: F \phi$. O tableau obtido por aplicação da regra $\left(T \neg^{\prime}\right)$ é, portanto, satisfeito pelo modelo.

Agora, suponha que o ramo $R$ de um tableau satisfatível possua um nó da forma $\sigma: \pi$ e que $|\sigma|<n$, de forma que a regra $\left(\pi^{\prime}\right)$ possa ser aplicada. Como $|\sigma|<n$, tem-se que $n-|\sigma|>0$. Serão analisados os dois casos:

1. $\pi=F \square \phi$ : Como existe um nó da forma $\sigma: F \square \phi$ no ramo que é satisfatível, tem-se que $\mathcal{M}, i(\sigma) \nvdash_{S, n-|\sigma|}^{3} \square \phi$. A aplicação da regra $\left(\pi^{\prime}\right)$ fornece um novo nó da forma $\sigma \cdot m: F \phi$ (em que $\sigma \cdot m$ é novo no ramo). Pela condição de verdade FWD5, tem-se que ou $n-|\sigma| \leq 0$ (falso) ou que existe $t \in W$ tal que $i(\sigma) R t$ e $\mathcal{M}, t \nvdash_{S, n-|\sigma|}^{3} \phi$. Logo, existe $t \in W$ tal que $i(\sigma) R t$ e $\mathcal{M}, t \nvdash_{S, n-|\sigma|}^{3} \phi$. Estenda a interpretação SST $i$ para uma outra $j$ como segue:

$$
j(s)=\left\{\begin{array}{l}
t \text { se } s=\sigma \cdot m \\
i(s) \text { nos outros casos }
\end{array}\right.
$$


Com isso, $\mathcal{M}, j(\sigma . m) \nvdash_{S, n-|\sigma|}^{3} \phi$, ou seja, $\mathcal{M}$ e $j$ satisfazem $\sigma . m: F \phi$; as outras sentenças do ramo são satisfeitas por $\mathcal{M}$ e $j$ justamente porque, nessas, $j$ é igual a $i$, que já as satisfazia anteriormente. Só resta provar que $j$ é de fato uma interpretação SST, conforme reza a Definição 6.9. Para todo prefixo $s$ diferente de $\sigma . m$ que ocorre no ramo, a função $j$ coincide com $i$, que já é uma interpretação SST. É preciso, portanto, apenas analisar o que ocorre com o novo prefixo introduzido no ramo. Por construção, $j(\sigma) R j(\sigma . m)$. Por fim, como $\sigma . m$ é novo no ramo, não existe $\sigma . m . l$ para o qual seja preciso verificar se $j(\sigma . m) R j(\sigma . m . l)$. Assim, neste caso o ramo $R$ é satisfatível por $\mathcal{M}$ com a interpretação SST $j$.

2. $\pi=T \diamond \phi$ : Como existe um nó da forma $\sigma: T \diamond \phi$ no ramo que é satisfatível, tem-se que $\mathcal{M}, i(\sigma) \models_{S, n-|\sigma|}^{3} \diamond \phi$. A aplicação da regra $\left(\pi^{\prime}\right)$ fornece um novo nó da forma $\sigma \cdot m: T \phi$ (em que $\sigma . m$ é novo no ramo). Pela condição de verdade FWD6, tem-se que ou $n-|\sigma| \leq 0$ (falso) ou que existe $t \in W$ tal que $i(\sigma) R t$ e $\mathcal{M}, t \models_{S, n-|\sigma|}^{3} \phi$. Logo, existe $t \in W$ tal que $i(\sigma) R t$ e $\mathcal{M}, t \models_{S, n-|\sigma|}^{3} \phi$. A extensão da função $i$ para outra $j$ e a verificação de que $j$ é uma interpretação SST que satisfaz, $\operatorname{com} \mathcal{M}$, todas as sentenças do ramo do tableau são idênticas às do caso anterior e não precisam ser repetidas.

Para provar a completude de um tableau, é suficiente mostrar que, se um ramo é aberto e todas as sentenças são reduzidas, então existe um contra-modelo à sentença (ou seqüente) que se deseja mostrar. O ramo aberto do tableau dá uma indicação de como construir esse contra-modelo. O primeiro passo, portanto, é descrever o que significa uma sentença ser reduzida em um ramo. Intuitivamente, é quando todas as regras que poderiam ser aplicadas o foram. Adaptando as definições da Seção 2.2 .2 para $\left\langle S_{3}, n\right\rangle$-SST, tem-se que uma sentença prefixada $\sigma: \varphi$ é reduzida em um ramo $R$ se:

R1. $\sigma: \varphi$ é um átomo assinalado

R2. $\sigma: \varphi$ é uma sentença $\sigma: \alpha$ e tanto $\sigma: \alpha_{1}$ como $\sigma: \alpha_{2}$ estão em $R$

R3. $\sigma: \varphi$ é uma sentença $\sigma:$ neg e $\sigma:$ pos está em $R$ (para a regra $(F \neg$ ), e para $\left(T \neg^{\prime}\right)$ quando $\sigma: \varphi$ é uma sentença $\sigma: T \neg \phi$ para $\phi \in S$ ); se $\sigma: \varphi$ é uma sentença $\sigma: T \neg \phi$ para $\phi \notin S$, ela já está reduzida em $R$

R4. $\sigma: \varphi$ é uma sentença $\sigma: \beta$ e ou $\sigma: \beta_{1}$ ou $\sigma: \beta_{2}$ está em $R$

R5. $\sigma: \varphi$ é uma sentença $\sigma: \pi$ e $\sigma \cdot m: \pi_{0}$ está em $R(\sigma . m$ não está previamente no ramo) quando $|\sigma| \leq n$; se $|\sigma|>n$, a sentença $\sigma: \pi$ já está reduzida para a regra $\left(\pi^{\prime}\right)$

R6. $\sigma: \varphi$ é uma sentença e, para todas as outras possíveis regras $R$ (que variam de lógica a lógica) e todo prefixo $\sigma^{*}$ em $R, \sigma^{*}: \varphi^{R}$ está em $R$ 
Comparando com as regras R1-6 da Seção 2.2.2, apenas as regras R3 e 5 são modificadas; essas são as regras não-clássicas de redução. Um tableau é construído, a grosso modo, reduzindo cada uma de suas sentenças para cada uma das regras, tentando fechá-lo. Se ele não fechar, existe a possibilidade de que um ramo tenha que ser infinito, para ser reduzido. Mas, como observado na Seção 2.2.2, Massacci [Mas00] apresenta técnicas que permitem a controlar tais ramos. Neste trabalho esses detalhes não serão analisados.

\begin{tabular}{|c|l|}
\hline Lógica & Condição sobre $\triangleright$ \\
\hline $\mathbf{K}$ & $\sigma \triangleright \sigma . n$ \\
$\mathbf{T}$ & $\sigma \triangleright \sigma . n$ e $\sigma \triangleright \sigma$ \\
$\mathbf{D}$ & $\sigma \triangleright \sigma . n$, desde que exista $n$ tal que $\sigma \triangleright \sigma . n$, \\
& ou $\sigma \triangleright \sigma$ \\
K4 & $\sigma \triangleright \sigma . \sigma^{\prime}$ com $\left|\sigma^{\prime}\right| \geq 1$ \\
K5 & $\sigma \triangleright \sigma . n$ e $\sigma . \sigma^{\prime} \triangleright \sigma . \sigma^{\prime \prime}$ com $\left|\sigma^{\prime}\right|,\left|\sigma^{\prime \prime}\right| \geq 1$ \\
KB & $\sigma \triangleright \sigma . n$ e $\sigma . n \triangleright \sigma$ \\
KDB & $\sigma \triangleright \sigma . n$ e $\sigma . n \triangleright \sigma$, desde que exista $n$ tal que \\
& $\sigma \triangleright \sigma . n$, ou $\sigma \triangleright \sigma$ \\
B & $\sigma \triangleright \sigma . n$ e $\sigma . n \triangleright \sigma$ e $\sigma \triangleright \sigma$ \\
KD4 & $\sigma \triangleright \sigma . \sigma^{\prime}$ com $\left|\sigma^{\prime}\right| \geq 1$, desde que exista $n$ tal \\
& que $\sigma \triangleright \sigma . n$, ou $\sigma \triangleright \sigma$ \\
K45 & $\sigma . \sigma^{\prime} \triangleright \sigma . \sigma^{\prime \prime} \operatorname{com}\left|\sigma^{\prime \prime}\right| \geq 1$ \\
KD5 & $\sigma \triangleright \sigma . n$ e $\sigma . \sigma^{\prime} \triangleright \sigma . \sigma^{\prime \prime}$ com $\left|\sigma^{\prime}\right|,\left|\sigma^{\prime \prime}\right| \geq 1$, desde \\
& que exista $n$ tal que $\sigma \triangleright \sigma . n$, ou $\sigma \triangleright \sigma$ \\
KD45 & $\sigma . \sigma^{\prime} \triangleright \sigma . \sigma^{\prime \prime}$ com $\left|\sigma^{\prime \prime}\right| \geq 1$, desde que exista $n$ \\
& tal que $\sigma \triangleright \sigma . n$, ou $\sigma \triangleright \sigma$ \\
S4 & $\sigma \triangleright \sigma . \sigma^{\prime}$ \\
KB4 & $\sigma \triangleright \sigma . \sigma^{\prime}$ e $\sigma . \sigma^{\prime} \triangleright \sigma$ com $\left|\sigma^{\prime}\right| \geq 1$ \\
S5 & $\sigma . \sigma^{\prime} \triangleright \sigma . \sigma^{\prime \prime}$ \\
\hline
\end{tabular}

Tabela 6.1: Lógicas modais e condições sobre a relação sintática $\triangleright$

Quando um ramo cujas sentenças estão todas reduzidas para todas as regras não fecha, intuitivamente a tentativa de encontrar uma contradição "falhou". Mas nem tudo se perde, porque - como se disse acima - o ramo dá informações suficientes para a construção de um contra-modelo à sentença que se desejava provar. Ramos assim são chamados de ramos abertos. Os objetos que são utilizados para a construção desse contra-modelo são os próprios prefixos, que fazem o papel de "mundos" do modelo ${ }^{3}$. A relação de acessibilidade é obtida a partir de uma relação sintática $\sigma \triangleright \sigma^{\prime}$ entre os prefixos (ver Tabela 6.1, adaptada de [Mas00]); isso varia de lógica a lógica, porque cada lógica é caracterizada por uma relação de acessibilidade. Massacci [Mas00] mostra como associar as relações de acessibilidade a relações sintáticas entre prefixos, mas como essas

\footnotetext{
${ }^{3} \mathrm{~A}$ origem dessas idéias para provar completude encontra-se, em última análise, nos trabalhos de Leon A. Henkin [Hen49]. Veja [Sch67] para a demonstração da completude da lógica de primeira ordem usando as idéias de Henkin.
} 
relações não dependem das regras que valem para todas as lógicas (e, a fortiori, das regras que foram modificadas para os $\left\langle S_{3}, n\right\rangle$-SST), elas não serão estudadas aqui. Por fim, a valoração dos átomos em cada mundo é, naturalmente, aquela fornecida pelas sentenças $\sigma: T p$ ( $p$ é verdadeiro no mundo $\sigma$ ) ou $\sigma: F p$ ( $p$ é falso no mundo $\sigma$ ). Agora é possível mostrar o

Teorema 6.12 (Completude) Suponha que $\Gamma_{G} \models_{S, n}^{3} \Gamma_{U} \Rightarrow \psi$, em que $\Gamma_{U}$ é o conjunto de hipóteses locais e $\Gamma_{G}$ o conjunto de hipóteses globais. Então o $\left\langle S_{3}, n\right\rangle$-SST para $\Gamma_{U} \cup \Gamma_{G} \vdash \psi$ fecha.

Dem: Seja $R$ um ramo aberto do tableau. Construa o modelo $\mathcal{M}=\left\langle W, R, v_{S}^{3}\right\rangle$ como segue:

- $W=\{\sigma: \sigma \in R\}$

- $\sigma R \sigma^{\prime}$ sse $\sigma \triangleright \sigma^{\prime}$

- $v_{S}^{3}(\sigma)(p)=1$ se $\sigma: T p \in R ; v_{S}^{3}(\sigma)(p)=0$ se $\sigma: F p \in R$

Observe que não há ambivalência na definição de $v_{S}^{3}(\sigma)(p)$ porque o ramo não fecha, ou seja, não ocorre simultaneamente $\sigma: T p$ e $\sigma: F p \in R$. Note também que a função $v_{S}^{3}$ ainda não foi totalmente definida; para átomos $q$ tais que $\sigma: T q$ ou $\sigma: F q$ não aparecem no ramo, assuma que uma determinada valoração foi fixada. Por fim, pelo Teorema 6.3, é preciso determinar a verdade das sentenças $\neg \phi(\sigma)$ tais que $\mathcal{M}, \sigma \models_{S, n-|\sigma|}^{3} \phi$ para todo $\sigma \in 1^{*}$. Assuma que $\mathcal{M}, \sigma \models_{S, n-|\sigma|}^{3}$ $\neg \phi$.

É necessário, agora, mostrar que o ramo $R$ é satisfatível por $\mathcal{M}$. Essa prova é por indução na complexidade das sentenças (a parte que fica à direita do prefixo) no ramo. Como na prova de correção, será analisada apenas a satisfatibilidade de $\mathcal{M}$ para sentenças da forma $T \neg \phi, \phi \notin S$ e para sentenças $\pi$ nos quais se utilizou a regra $\left(\pi^{\prime}\right)$.

Se há uma sentença prefixada da forma $\sigma: T \neg \phi, \phi \notin S$, pela condição R3 ela está reduzida. Pela hipótese acima, sentenças dessa forma são automaticamente satisfeitas (já que todo prefixo no ramo é $R$-ligado a 1). Portanto, $\mathcal{M}, \sigma \models_{S, n-|\sigma|}^{3}$ $\neg \phi$.

Suponha agora que haja no ramo uma sentença da forma $\sigma: \pi$ e que ela é reduzida segundo a regra $\left(\pi^{\prime}\right)$. Há dois casos a considerar. No primeiro caso, $\sigma: \pi$ é reduzida porque existe no ramo uma sentença da forma $\sigma . m: \pi_{0}$. Nesse caso, só pode ser $|\sigma|<n$ (senão a regra não poderia ter sido aplicada). Então $n-|\sigma|>0$. Dois subcasos:

1. $\pi=F \square \phi:$ Então, deve ocorrer $\sigma . m: F \phi \in R$. Pela hipótese indutiva, tem-se que $\mathcal{M}, \sigma .\left.m\right|_{S, n-|\sigma . m|} ^{3} \phi$. Por construção, $\sigma R \sigma . m$. Portanto, pela condição de verdade FWD5, $\mathcal{M}, \sigma \nvdash_{S, n-|\sigma|}^{3} \square \phi$.

2. $\pi=T \diamond \phi$ : Então, deve ocorrer $\sigma \cdot m: T \phi \in R$. Pela hipótese indutiva, tem-se que $\mathcal{M}, \sigma . m \models_{S, n-|\sigma . m|}^{3} \phi$. Por construção, $\sigma R \sigma . m$. Portanto, pela condição de verdade FWD6, $\mathcal{M}, \sigma \models_{S, n-|\sigma|}^{3} \diamond \phi$. 
No segundo caso, $\sigma: \pi$ é reduzida porque $|\sigma| \geq n$. Isso implica que $n-$ $|\sigma| \leq 0$. Mas, pelas condições de verdade FWD5 (respectivamente, FWD6), se $\pi=F \square \phi$ (respectivamente, $\pi=T \diamond \phi)$, necessariamente $\mathcal{M}, \sigma \nvdash_{S, n-|\sigma|}^{3} \square \phi$ (respectivamente, $\mathcal{M}, \sigma \models_{S, n-|\sigma|}^{3} \diamond \phi$ ).

$\mathrm{O}$ algoritmo sugerido pelo procedimento apresentado para a obtenção de uma prova clássica de uma sentença é o seguinte:

1. $S:=\emptyset, n:=0$

2. transforme o seqüente de entrada em um $\left\langle S_{3}, n\right\rangle$-SST inicial

3. expanda o tableau até que ele feche ou fique bloqueado (como ocorreu nos exemplos acima nas interrogações)

4. se o tableau fechar, terminar com sucesso

5. se o tableau contiver um ramo que não puder ser classicamente expandido, terminar com falha

6. se o tableau ficar bloqueado devido a uma sentença $T \neg \phi$, faça $S:=S \cup\{\phi\}$ e volte ao passo 3

7. se o tableau fechar devido à aplicação da regra (bey), faça $n:=n+1 \mathrm{e}$ volte ao passo 3

\subsection{Semântica e Teoria de Prova Alternativos}

Um aspecto da teoria da prova apresentada na Seção 6.2 é a existência de uma regra não-clássica de fechamento, sempre que se chega a uma sentença $\sigma: \nu$ cujo prefixo supera determinado comprimento; a regra (bey) tornou-se necessária para a correção e completude dos $\left\langle S_{3}, n\right\rangle$-SST com relação à semântica FWD18. Esta seção apresenta uma semântica que contorna a necessidade dessa regra.

Seja $\mathcal{M}=\left\langle W, R, v_{S}^{3}\right\rangle$ um modelo $S_{3}$-Kripke e $n$ o limite máximo de introspecção, e considere a semântica dada pelas regras FWE1-8 abaixo ( $p^{\circ}$ é um literal):

FWE1. $\mathcal{M}, w \models_{S, n}^{3^{\prime}} p^{\circ}$ sse $v_{S}^{3}(w)\left(p^{\circ}\right)=1$

FWE2. $\mathcal{M}, w \models_{S, n}^{3^{\prime}} \phi \wedge \psi$ sse $\mathcal{M}, w \models_{S, n}^{3^{\prime}} \phi$ e $\mathcal{M}, w \models_{S, n}^{3^{\prime}} \psi$

FWE3. $\mathcal{M}, w \models_{S, n}^{3^{\prime}} \phi \vee \psi$ sse $\mathcal{M}, w \models_{S, n}^{3^{\prime}} \phi$ ou $\mathcal{M}, w \models_{S, n}^{3^{\prime}} \psi$

FWE4. $\mathcal{M}, w \models_{S, n}^{3^{\prime}} \phi \supset \psi$ sse $\mathcal{M}, w \models_{S, n}^{3^{\prime}} \phi$ ou $\mathcal{M}, w \models_{S, n}^{3^{\prime}} \psi$

FWE5. se $n>0, \mathcal{M}, w \models_{S, n}^{3^{\prime}} \square \phi$ sse para todo $t \in W$ tal que $w R t, \mathcal{M}, t \models_{S, n-1}^{3^{\prime}}$ $\phi$

FWE6. se $n>0, \mathcal{M}, w \models_{S, n}^{3^{\prime}} \diamond \phi$ sse existe $t \in W$ tal que $w R t$ e $\mathcal{M}, t \models_{S, n-1}^{3^{\prime}} \phi$ 
FWE7. se $n \leq 0$, ou $\mathcal{M}, w \models=_{S, n}^{3^{\prime}} \square \phi$ ou $\mathcal{M}, w \nvdash_{S, n}^{3^{\prime}} \square \phi$

FWE8. se $n \leq 0$, ou $\mathcal{M}, w \models=_{S, n}^{3^{\prime}} \diamond \phi$ ou $\mathcal{M}, w \not_{S, n}^{3^{\prime}} \diamond \phi$

FWE9. se $\phi \in S, \mathcal{M}, w \models_{S, n}^{3^{\prime}} \neg \phi$ sse $\mathcal{M}, w \not \nvdash_{S, n}^{3^{\prime}} \phi$

FWE10. não acontece simultaneamente que $\mathcal{M}, w \forall_{S, n}^{3^{\prime}} \phi$ e $\mathcal{M}, w \nvdash_{S, n}^{3^{\prime}} \neg \phi$

Esta semântica será denotada por $\left\langle S_{3}, n\right\rangle^{\prime}$, para não haver confusão com a semântica dada na Seção 6.1. Os conceitos de $\left\langle S_{3}, n\right\rangle^{\prime}$-satisfatibilidade, $\left\langle S_{3}, n\right\rangle^{\prime}$ validade e $\left\langle S_{3}, n\right\rangle^{\prime}$-insatisfatibilidade modais são definidos como usualmente.

Observa-se uma peculiaridade nas cláusulas FWE7-8 (que é justamente o que faz com que FWE1-10 seja distinta de FWD1-8). Nessas cláusulas, não há nenhuma restrição à semântica dos operadores $\square$ e $\diamond$. Uma necessitação ou possibilitação pode ser ou não aceita, de maneira arbitrária.

Intuitivamente, isso quer dizer que, além do limite máximo de introspecção, uma proposição pode não ser necessária em um mundo mesmo se ela for verdadeira em todos os mundos que ele acessar, e uma proposição pode ser possível em um mundo mesmo se ela for falsa para todos os mundos que ele acessa. Esse comportamento aparentemente paradoxal pode ser entendido, de maneira figurada, como segue. Um limite introspectivo expressa o caráter finito da capacidade que um agente pensante tem de raciocinar sobre as crenças de outros agentes; além desse limite, o agente não consegue raciocinar. $O$ que ele faz, então, é atribuir valores arbitrários às crenças dos agentes muito distantes, sem se preocupar com a consistência dessas crenças. Isso se assemelha à "leitura rasa" das crenças explícitas de Levesque (Seção 1.1).

Alguns teoremas saem da definição da semântica.

Teorema 6.13 $A\left\langle S_{3}, n\right\rangle^{\prime}$-verdade de uma sentença $\psi$ para um mundo $w$ é determinada por $v_{S}^{3}$ e pelos conjuntos

$$
\begin{aligned}
& A=\left\{\neg \phi(t, j) \text { para todo } t \in w^{*} \text { tal que } \mathcal{M}, t \models_{S, j}^{3^{\prime}} \phi, \phi \notin S, j \leq n\right\}, \\
& B=\left\{\square \phi(t, j) \text { para todo } t \in w^{*}, j \leq 0\right\} \\
& C=\left\{\oslash \phi(t, j) \text { para todo } t \in w^{*}, j \leq 0\right\}
\end{aligned}
$$

É possível se restringir às sentenças $\neg \phi, \square \phi e \diamond \phi$ que são subsentenças de $\psi$. As notações $\neg \phi(t, j), \square \phi(t, j)$ e $\diamond \phi(t, j)$ significam que a verdade de $\neg \phi, \square \phi$ $e \diamond \phi$, respectivamente, devem ser avaliadas no mundo $t \in W$ para o limite de introspecção $j$.

Dem: Esse teorema é similar ao do Teorema 6.3. Para os conectivos $\wedge, \vee, \supset$, é imediato que a $\left\langle S_{3}, j\right\rangle^{\prime}$-verdade de $\phi$ é determinada pela das subsentenças para $w$ e para todos os outros $t \in W R$-ligados a $w$. Quando $j>0$, a $\left\langle S_{3}, j\right\rangle^{\prime}$-verdade (ou falsidade) de $\square \phi$ é equivalente à $\left\langle S_{3}, j-1\right\rangle^{\prime}$-verdade (ou falsidade) de que para todo $t \in W$ tal que $w R t$, ocorre $\mathcal{M}, t \models_{S, n-1}^{3^{\prime}} \phi$ (cláusula FWE5). Quando $j \leq 0$, então a $\left\langle S_{3}, j\right\rangle^{\prime}$-verdade de $\square \phi$ é arbitrária e precisa ser determinada (cláusula FWE7). Considerações similares valem para $\diamond \phi$ (cláusulas FWE6 e 
8). A determinação por $v_{S}^{3}$ e $A$ é demonstrada exatamente da mesma maneira que no Teorema 6.3.

O teorema acima permite a elaboração do conceito de tradução de uma sentença. Aqui, a tradução depende do mundo $t \in W$ em que se está avaliando a verdade, bem como de $n$. Assim, seja

- $p_{\neg \phi, t, j}$ um novo átomo para todo $t \in w^{*}$ tal que $\mathcal{M}, t \models_{S, j}^{3^{\prime}} \phi, \phi \notin S$ e $j \leq n$, e cuja valoração é verdadeira se $\mathcal{M}, t \models_{S, n}^{3^{\prime}} \neg \phi$ e falsa caso contrário

- $p_{\square \phi, t, j}$ um novo átomo para todo $t \in w^{*}$ e $j \leq 0$, cuja valoração é verdadeira se $\mathcal{M}, t \models_{S, j}^{3^{\prime}} \square \phi$, e falsa caso contrário

- $p_{\diamond \phi, t, j}$ um novo átomo para todo $t \in w^{*}$ e $m \leq 0$, cuja valoração é verdadeira se $\mathcal{M}, t \models_{S, j}^{3^{\prime}} \diamond \phi$, e falsa caso contrário

e defina a tradução $\phi^{\bullet S, t, n}$ de $\phi$ da seguinte maneira:

$$
\begin{aligned}
& p^{\bullet S, t, n}=p \\
& (\phi \wedge \psi)^{\bullet S, t, n}=\phi^{\bullet S, t, n} \wedge \psi^{\bullet S, t, n} \\
& (\phi \vee \psi)^{\bullet S, t, n}=\phi^{\bullet S, t, n} \vee \psi^{\bullet S, t, n} \\
& (\phi \supset \psi)^{\bullet S, t, n}=\phi^{\bullet S, t, n} \supset \psi^{\bullet S, t, n} \\
& (\square \phi)^{\bullet S, t, n}= \begin{cases}\square\left(\phi^{\bullet S, t, n}\right) & \text { se } n>0 \\
p_{\square \phi, t, n} & \text { caso contrário }\end{cases} \\
& (\diamond \phi)^{\bullet S, t, n}= \begin{cases}\left.\diamond^{\bullet} \phi^{\bullet S, t, n}\right) & \text { se } n>0 \\
p_{\diamond \phi, t, n} & \text { caso contrário }\end{cases} \\
& (\neg \phi)^{\bullet S, t, n}= \begin{cases}\neg\left(\phi^{\bullet S, t, n}\right) & \text { se } \phi \in S \text { ou se } \mathcal{M}, t \not \nvdash_{S, n}^{3^{\prime}} \phi \\
p_{\neg \phi, t, n} & \text { caso contrário }\end{cases}
\end{aligned}
$$

Os dois teoremas a seguir são equivalentes ao Teorema 6.4 e ao Teorema 6.5 , respectivamente. As demonstrações são semelhantes, com as adaptações evidentes por causa da adição dos átomos $p_{\square \phi, t, n}$ e $p_{\diamond \phi, t, n}$, e não serão dadas aqui.

Teorema 6.14 Uma sentença $\phi$ é $\left\langle S_{3}, n\right\rangle^{\prime}$-satisfativel em algum mundo $w$ de um modelo $\mathcal{M}$ sse $\phi^{\circ S, w}$ é satisfativel classicamente para $w$ em $\mathcal{M}$.

Teorema 6.15 Seja Prop $(\phi)$ o conjunto dos átomos de $\phi$. Toda sentença $\phi$ com $\operatorname{Prop}(\phi) \cap S=\emptyset$ é $\left\langle S_{3}, n\right\rangle^{\prime}$-satisfativel.

Monotonicidade e convergência valem, e pelas mesmas razões.

Seguem alguns exemplos; neles, supõe-se que $S=A$, ou seja, que o conjunto de proposições relevantes é igual ao conjunto de átomos:

1. Necessitação não vale em uma aproximação $\left\langle S_{3}, n\right\rangle^{\prime}$-Kripke. Seja $\mathcal{M}$ um modelo qualquer, $w$ um mundo desse modelo e tome $n=0$. Suponha que $\phi$ seja válida (por exemplo, uma tautogia), de forma que $\mathcal{M}, t \models_{S, n}^{3^{\prime}} \phi$ seja 
qual forem $t$ e $n$. Pelo Teorema 6.13, como $n \leq 0$, é possível escolher a verdade de $\square \phi$ em $w$ para o limite de introspecção $n$. Assim, assuma que $\mathcal{M}, w \not \not_{S, 0}^{3^{\prime}} \square \phi$.

2. Não vale o princípio $K: \models_{S, n}^{3^{\prime}} \square(p \supset q) \supset(\square p \supset \square q)$. Seja $n=0$. Considere o modelo $\mathcal{M}=\left\langle W, R, v_{S}^{3}\right\rangle$ e um mundo $w$ desse modelo. Como no exemplo anterior, como $n \leq 0$, pelo Teorema 6.13 é possível escolher a verdade de $\square \phi$ em $w$ para o limite de introspecção $n$. Assim, pode-se escolher a verdade de $\square p$, bem como de $\square q$ e $\square(p \supset q)$, em $w$; fazendo $\mathcal{M}, w \models_{S, 0}^{3} \square(p \supset q), \mathcal{M}, w \models_{S, 0}^{3} \square p$ e $\mathcal{M}, w \nvdash_{S, 0}^{3} \square q$, obtém-se um contra-modelo para $K$.

3. Não vale a equivalência $\diamond \phi \equiv \neg \square \neg \phi$. Seja $n=0$. Considere o modelo $\mathcal{M}=\left\langle W, R, v_{S}^{3}\right\rangle$ e um mundo $w$ desse modelo. Pelo Teorema 6.13, como $n \leq 0$, pode-se escolher a verdade de $\square p$ em $w$. Assuma que $\mathcal{M}, w \forall_{S, 0}^{3} \square p$. Pelo mesmo teorema, pode-se escolher a verdade de $\diamond \neg p$

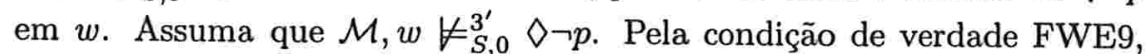
ocorre $\mathcal{M}, w \models{ }_{S, 0}^{3^{\prime}} \neg \diamond \neg p$. Adaptações simples desse modelo mostra que não valem nenhuma das outras equivalências $\neg \square \phi \equiv \diamond \neg \phi, \neg \square \neg \phi \equiv \diamond \phi$.

Os exemplos 1 e 2 acima mostram que a lógica não é normal, porque não vale nem a Regra da Necessitação nem $K$; e, por não valer $K$, essa semântica difere das apresentadas na Seção 6.1.

Os tableaux de prova (que serão chamados de $\left\langle S_{3}, n\right\rangle^{\prime}$-SST) são mais simples que os $\left\langle S_{3}, n\right\rangle$-SST. De fato, tudo é exatamente igual, com a diferença que, desta vez, não há a regra de fechamento (bey). Assim, segue a

Definição 6.16 Tableaux obtidos utilizando as regras $\left(T \neg^{\prime}\right)$ e $\left(\pi^{\prime}\right)$ e as outras regras SST sem alteração, apenas com a regra de fechamento clássica, são chamados de $\left\langle S_{3}, n\right\rangle^{\prime}$-SST $\left(\left\langle S_{3}, n\right\rangle^{\prime}\right.$-tableaux passo a passo).

A demonstração da correção dos $\left\langle S_{3}, n\right\rangle^{\prime}$-SST é exatamente igual, linha a linha, àquela apresentada na Seção 6.2 (o que não é inesperado, pois as regras de expansão são idênticas), e não será repetida. Naturalmente, o conceito de interpretação SST e de satisfatibilidade de um ramo do tableau por um modelo são as mesmas.

A demonstração da completude é quase a mesma também, mas precisa de uma pequena modificação. Pelo Teorema 6.13 , é preciso determinar a verdade das sentenças

- $\neg \phi(\sigma, n-|\sigma|)$ tais que $\mathcal{M}, \sigma \models \models_{S, n-|\sigma|}^{3^{\prime}} \phi$ para todo $\sigma \in 1^{*}$ : considere que $\mathcal{M}, \sigma \models{ }_{S, n-|\sigma|}^{3^{\prime}} \neg \phi$

- $\square \phi(\sigma, n-|\sigma|)$ quando $n-|\sigma| \leq 0$ para todo $\sigma \in 1^{*}$ : considere que $\mathcal{M}, \sigma \models \models_{S, n-|\sigma|}^{3^{\prime}} \square \phi$ se $\sigma: T \square \phi$ está no ramo, e $\mathcal{M}, \sigma \nvdash_{S, n-|\sigma|}^{3^{\prime}} \square \phi$ se $\sigma: F \square \phi$ está no ramo 
- $\diamond \phi(\sigma, n-|\sigma|)$ quando $n-|\sigma| \leq 0$ para todo $\sigma \in 1^{*}$ : considere que $\mathcal{M}, \sigma \models_{S, n-|\sigma|}^{3^{\prime}} \diamond \phi$ se $\sigma: T \diamond \phi$ está no ramo, e $\mathcal{M}, \sigma \nvdash_{S, n-|\sigma|}^{3^{\prime}} \diamond \phi$ se $\sigma: F \diamond \phi$ está no ramo

A segunda cláusula acima implica que, se $\sigma: F \square \phi$ aparece no tableau para $|\sigma| \geq n$, não há nada que force $\mathcal{M}$ a não satisfazer $\phi$ para todo $\sigma . m$ diretamente acessível de $\sigma$. Da mesma forma, a terceira cláusula acima implica que, se $\sigma: T \diamond \phi$ aparece no tableau para $|\sigma| \geq n$, não há nada que force $\mathcal{M}$ a satisfazer $\phi$ para algum $\sigma . m$ diretamente acessível de $\sigma$. Com isso, quando $|\sigma| \geq n$ a regra $(\pi)$ não pode mesmo ser aplicada, já que existem modelos que satisfazem sua hipótese mas não sua conclusão. Por outro lado, não há nada contraditório na existência de uma sentenças da forma $\sigma: T \square \phi$ e $\sigma: F \diamond \phi,|\sigma| \geq n$, no tableau: a semântica permite que isso ocorra.

Com isso, obtém-se tableaux de prova consideravelmente simples e flexíveis, sem a existência de uma nova regra para fechamento. A semântica foi obtida por um enfraquecimento de FWD1-8. A seguir apresentam-se exemplos de aplicação $\operatorname{dos}\left\langle S_{3}, n\right\rangle^{\prime}$-SST.

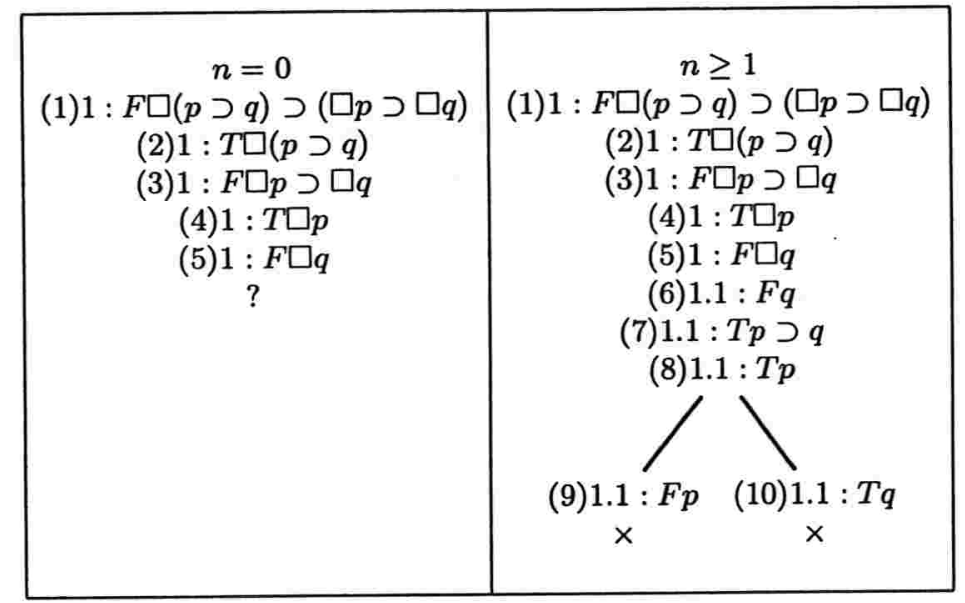

Figura 6.6: Prova de $K$ por $\left\langle S_{3}, n\right\rangle^{\prime}$-SST

Na Figura 6.6 há uma prova de $K$. No lado esquerdo, fixou-se o limite máximo de introspecção $n=0 \mathrm{e}$, no lado direito, $n \geq 1$. As sentenças (2)-(3) (dos dois lados) são obtidas de (1) pela regra $\alpha$. As sentenças (4)-(5) (dos dois lados também), de (3) por $\alpha$ também. No lado esquerdo, como $n=0$, não é possível aplicar a regra $\left(\pi^{\prime}\right)$, já que $|1|=0$, que não é menor que o limite máximo de introspecção 0 . No lado direito, como essa condição pode ser satisfeita (isto é, $|1|=0<1$ ), prossegue-se a demonstração. As sentenças (7) e (8) são obtidas de (2) e (4), respectivamente, por aplicação da regra $(K)$. Por fim, as sentenças (9)-(10) são obtidas de (7) por $\beta$.

O lado esquerdo dá um contra-modelo à sentença que se desejava provar. Como $n \leq 0$, é possível escolher a valoração de $\square(p \supset q), \square p$ e $\square q$ para o mundo 1 e $n=0$. Assuma que $\mathcal{M}, 1 \models_{S, 0}^{3^{\prime}} \square(p \supset q), \mathcal{M}, 1 \models_{S, 0}^{3^{\prime}} \square p$ e $\mathcal{M}, 1 \nvdash_{S, 0}^{3^{\prime}} \square q$. 


\begin{tabular}{|c|c|}
\hline & $n \geq 0$ \\
$(1) 1: F \square p \supset \square \square p$ & $(1) 1: F \square p \supset \square \square p$ \\
$(2) 1: T \square p$ & $(2) 1: T \square p$ \\
$(3) 1: F \square \square p$ & $(3) 1: F \square \square p$ \\
$(4) 1: T p$ & $(4) 1: T p$ \\
$?$ & $(5) 1.1: F \square p$ \\
& $(6) 1.1: T \square p$ \\
& $\times$ \\
\hline
\end{tabular}

Figura 6.7: Prova de $\square p \supset \square \square p$ na lógica modal S4 por $\left\langle S_{3}, n\right\rangle^{\prime}$-SST

Na. Figura 6.7 há uma prova de $\square p \supset \square \square p$ na lógica modal S4. Essa lógica possui, em adição aos princípios válidos para todas as lógicas, as regras $(K)$, $(T)$ e (4). No lado esquerdo, fixou-se o limite máximo de introspecção $n=0$ e, no lado direito, $n \geq 1$. As sentenças (2)-(3) (dos dois lados) são obtidas de (1) pela regra $\alpha$, e a sentença (4) (nos dois lados também) é obtida de (2) por $(T)$. No lado esquerdo, como $n=0$, não é possível aplicar a regra $\left(\pi^{\prime}\right)$ à sentença $(4)$, já que $|1|=0$, que não é menor que o limite máximo de introspecção 0 . No lado direito, como essa condição pode ser satisfeita (isto é, $|1|=0<1$ ), prossegue-se a demonstração. A sentença (6) é obtida de (2) por aplicação da regra $(T)$. Especialmente interessante nessa demonstração é o fato de que, em alguns sistemas axiomáticos $\mathbf{S} 4$, o princípio $\square p \supset \square \square p$ é tomado como axioma. A demonstração apresentada no exemplo torna evidente que é necessária a tomada de pelo menos um passo introspectivo para concluir a verdade desse princípio. A caracterização de S4 por meio de regras SST é mais forte do que a caracterização usual por sistemas de Hilbert, no sentido que certos princípios que são axiomáticos para os últimos (e, portanto, não exigem análise a posteriori) podem ser demonstrados para os primeiros.

O lado esquerdo dá um contra-modelo à sentença que se desejava provar. Observe que, como $1: T p$ está no ramo, tem-se $v_{S}^{3}(1)(p)=1 \mathrm{e}$, em particular, $\mathcal{M}, 1 \models_{S, 0}^{3^{\prime}} p$. Como $n \leq 0$, é possível escolher a valoração de $\square p$ e $\square \square p$ para o mundo 1 e $n=0$. Assuma que $\mathcal{M}, 1 \models_{S, 0}^{3^{\prime}} \square p$ e $\mathcal{M}, 1 \not \models_{S, 0}^{3^{\prime}} \square \square p$.

Na Figura 6.8 há uma prova de $\square p \supset \square \square \square p$ na lógica modal S5. Essa lógica possui, em adição aos princípios válidos para todas as lógicas, as regras $(K)$ e $(T)$. No lado esquerdo, fixou-se o limite máximo de introspecção $n=0$, no meio fixou-se $n=1$ e, no lado direito, $n \geq 2$. As sentenças (2)-(3) (em todas as colunas) é obtida de (1) pela regra $\alpha$, e a sentença (4) (em todas as colunas também) é obtida de (2) por $(T)$. No lado esquerdo, como $n=0$, não é possível aplicar a regra $\left(\pi^{\prime}\right)$ à sentença $(3)$. No meio e no lado direito, como a regra pode ser aplicada, prossegue-se a demonstração. A sentença (6) (no meio e no lado direito) é obtida de (2) por aplicação da regra (4), e a sentença (7), nesses dois lados, é obtida de (6) por $(T)$. Não é mais possível prosseguir no meio, mas novamente aplica-se a regra $\left(\pi^{\prime}\right)$ a (5) no lado direito. Por fim, a sentença (9) é obtida de (6) pela regra (4). Esse exemplo mostra que, quanto maior o 


\begin{tabular}{|c|c|c|}
\hline$n=0$ & $n=1$ & $n \geq 2$ \\
$(1) 1: F \square p \supset \square \square \square p$ & $(1) 1: F \square p \supset \square \square \square p$ & $(1) 1: F \square p \supset \square \square \square p$ \\
$(2) 1: T \square p$ & $(2) 1: T \square p$ & $(2) 1: T \square p$ \\
$(3) 1: F \square \square \square p$ & $(3) 1: F \square \square \square p$ & $(3) 1: F \square \square \square p$ \\
$(4) 1: T p$ & $(4) 1: T p$ & $(4) 1: T p$ \\
$?$ & $(5) 1.1: F \square \square p$ & $(5) 1.1: F \square \square p$ \\
& $(6) 1.1: T \square p$ & $(6) 1.1: T \square p$ \\
& $(7) 1.1: T p$ & $(7) 1.1: T p$ \\
& $?$ & $(8) 1.1 .1: F \square p$ \\
& & $(9) 1.1 .1: T \square p$ \\
& & $\times$ \\
& & \\
\hline
\end{tabular}

Figura 6.8: Prova de $\square p \supset \square \square \square p$ na lógica modal S5 por $\left\langle S_{3}, n\right\rangle^{\prime}$-SST

número de interações modais nas sentenças, maior deve ser o limite máximo de introspecção para que se possa demonstrá-las.

O lado esquerdo dá um contra-modelo à sentença que se desejava provar. Como $n \leq 0$, é possível escolher a valoração de $\square p$ e $\square \square \square p$ para o mundo $1 \mathrm{e}$ $n=0$. Assuma que $\mathcal{M}, 1 \models_{S, 0}^{3^{\prime}} \square p$ e $\mathcal{M}, 1 \nvdash_{S, 0}^{3^{\prime}} \square \square \square p$.

No meio, tem-se que $\mathcal{M}, 1 \models_{S, 1}^{3^{\prime}} p, \mathcal{M}, 1 \models_{S, 0}^{3^{\prime}} p$ e $\mathcal{M}, 1.1 \models_{S, 0}^{3^{\prime}} p$ (já que $v_{S}^{3}(1)(p)=v_{S}^{3}(1.1)(p)=1$ - as sentenças $1: T p$ e $1.1: T p$ estão no ramo). Como o prefixo 1.1 é tal que $|1.1|=1 \leq n$, é possível escolher a valoração de $\square p$ e $\square \square p$ em 1.1, para $n=0$. Assuma que $\mathcal{M}, 1.1 \models_{S, 0}^{3^{\prime}} \square p$ e $\mathcal{M}, 1.1 \nvdash_{S, 0}^{3^{\prime}}$ $\square \square p$. Pela Tabela 6.1, a relação de acessibilidade entre os prefixos é dada por $\sigma . \sigma^{\prime} R \sigma . \sigma^{\prime \prime}$; portanto, $1 R 1,1 R 1.1,1.1 R 1$ e $1.1 R 1.1$. Para todo mundo $t$ que 1 acessa (ou seja, para $t=1$ e $t=1.1$ ), tem-se que $\mathcal{M}, t \models_{S, 1-(|1|+1)}^{3^{\prime}} p$. Portanto, $\mathcal{M}, 1 \models_{S, 1}^{3^{\prime}} \square p$. Por outro lado, como $\mathcal{M}, 1.1 \nvdash_{S, 0}^{3^{\prime}} \square \square p$ e 1 acessa 1.1 , tem-se que $\mathcal{M}, 1 \not \nvdash_{S, 1}^{3^{\prime}} \square \square \square p$.

Observe, por fim, que o exemplo da Figura 6.5 funciona da mesma maneira, e pelas mesmas razões, com esta teoria de prova, já que, ali, a regra de fechamento não-clássica não é utilizada.

Uma pergunta cabível é: qual das duas semânticas, a $\left\langle S_{3}, n\right\rangle$ ou $\left\langle S_{3}, n\right\rangle^{\prime}$, consegue provar mais teoremas? Na semântica $\left\langle S_{3}, n\right\rangle$, a verdade de $\square \phi$ está sempre determinado quando $n \leq 0$, bem como o valor de $\diamond \phi$. Isso, como foi visto, não ocorre para $\left\langle S_{3}, n\right\rangle^{\prime}$. Portanto, dada uma valoração nos átomos, há mais modelos para $\left\langle S_{3}, n\right\rangle^{\prime}$ que para $\left\langle S_{3}, n\right\rangle$ (há mais liberdade de escolhas para a semântica desta seção). E quanto mais modelos uma certa semântica tem, menos teoremas ela pode provar, já que os teoremas precisam ser válidos em todos os modelos. Assim, a semântica $\left\langle S_{3}, n\right\rangle^{\prime}$ prova menos teoremas. Isso não é inesperado, porque as teorias de prova de ambas semânticas são idênticas a menos de uma regra de fechamento a mais na $\left\langle S_{3}, n\right\rangle$.

$\mathrm{O}$ algoritmo sugerido pelo procedimento apresentado para a obtenção de uma prova clássica de uma sentença é o seguinte: 
1. $S:=\emptyset, n:=0$

2. transforme o seqüente de entrada em um $\left\langle S_{3}, n\right\rangle^{\prime}$-SST inicial

3. expanda o tableau até que ele feche ou fique bloqueado (como ocorreu nos exemplos acima nas interrogações)

4. se o tableau fechar, terminar com sucesso

5. se o tableau contiver um ramo que não puder ser classicamente expandido, terminar com falha

6. se o tableau ficar bloqueado devido a uma sentença $T \neg \phi$, faça $S:=S \cup\{\phi\}$ e volte ao passo 3

7. se o tableau ficar bloqueado devido ao fato que $|\sigma| \leq n$, faça $n:=n+1$ e volte ao passo 3

\subsection{Uma Semântica mais Geral}

A razão de se ter, na semântica FWE1-10, determinado um limite máximo de introspecção $n$, além do qual necessitações e possibilitações se comportam de maneira não-clássica, foi motivado por considerações filosóficas. Um agente pensante finito possui uma capacidade máxima de introspecção; ele não consegue raciocinar completamente sobre as crenças de agentes que ficam além desse limite, e atribui valores arbitrários a algumas. Matematicamente, qualquer outra condição que pudesse simultaneamente ser utilizada na semântica e nos SST pode ser aplicada. A melhor maneira de controlar a prova por SST é solicitando alguma condição sobre os prefixos. Uma possível condição é um tamanho máximo para os mesmos. Outra possível condição é solicitar que os prefixos que apareçam sejam compostos apenas por números ímpares (ainda que isso nada acrescente de interessante). Ainda outra condição pode ser que o comprimento dos prefixos seja um número par.

Um prefixo $\sigma$ codifica o número de passos introspectivos necessários para se chegar da sentença ou seqüente inicial, que se tenta falsear no mundo "real" denotado por 1, à sentença que deve ser verdadeira no mundo denotado por $\sigma$. Cada seqüência de prefixos $1,1 . a_{1}, 1 . a_{1} . a_{2}, \ldots, 1 . a_{1} . a_{2} \ldots . . a_{k}$ é associada, portanto, a uma sequêencia de mundos $w_{0}, w_{1}, w_{2}, \ldots, w_{k}$, de forma que, para $1 \leq i \leq k$, tenha-se $w_{i-1} R w_{i}$. Essa seqüência de mundos será denominada caminho de $w_{0} a w_{k}$ e denotada $\mathbb{P}\left(w_{0}, \ldots, w_{k}\right)$. O comprimento do caminho, denotado por $\left|\mathbb{P}\left(w_{0}, \ldots, w_{k}\right)\right|$ é dado pelo número de elementos da sequiência, no caso $k+1$. A maneira geral de se denotar uma certa condição sobre um caminho $\mathbb{P}\left(w_{0}, \ldots, w_{k}\right)$ será escrevendo $\operatorname{Cond}\left(\mathbb{P}\left(w_{0}, \ldots, w_{k}\right)\right)$.

Com essas notações, é possivel apresentar uma semântica mais geral que a FWE1-10 da Seção 6.3. Ela é dada pelas regras abaixo:

FWG1. $\mathcal{M}, \mathbb{P}\left(w_{0}, \ldots, w_{k}\right) \models_{S}^{3} p^{\circ}$ sse $v_{S}^{3}\left(w_{0}\right)\left(p^{\circ}\right)=1$ 
FWG2. $\mathcal{M}, \mathbb{P}\left(w_{0}, \ldots, w_{k}\right) \models_{S}^{3} \phi \wedge \psi$ sse $\mathcal{M}, \mathbb{P}\left(w_{0}, \ldots, w_{k}\right) \models_{S}^{3} \phi$ e $\mathcal{M}, \mathbb{P}\left(w_{0}, \ldots, w_{k}\right) \models_{S}^{3} \psi$

FWG3. $\mathcal{M}, \mathbb{P}\left(w_{0}, \ldots, w_{k}\right) \models_{S}^{3} \phi \vee \psi$ sse $\mathcal{M}, \mathbb{P}\left(w_{0}, \ldots, w_{k}\right) \models_{S}^{3} \phi$ ou $\mathcal{M}, \mathbb{P}\left(w_{0}, \ldots, w_{k}\right) \models_{S}^{3} \psi$

FWG4. $\mathcal{M}, \mathbb{P}\left(w_{0}, \ldots, w_{k}\right) \models_{S}^{3} \phi \supset \psi$ sse $\mathcal{M}, \mathbb{P}\left(w_{0}, \ldots, w_{k}\right) \not \models_{S}^{3} \phi$ ou $\mathcal{M}, \mathbb{P}\left(w_{0}, \ldots, w_{k}\right) \models_{S}^{3} \psi$

FWG5. se $\operatorname{Cond}\left(\mathbb{P}\left(w_{0}, \ldots, w_{k}\right)\right), \mathcal{M}, \mathbb{P}\left(w_{0}, \ldots, w_{k}\right) \models_{S}^{3} \square \phi$ sse para todo $w_{m+1} \in W$ tal que $w_{k} R w_{m+1}, \mathcal{M}, \mathbb{P}\left(w_{0}, \ldots, w_{k}, w_{m+1}\right) \models_{S}^{3} \phi$

FWG6. se $\operatorname{Cond}\left(\mathbb{P}\left(w_{0}, \ldots, w_{k}\right)\right), \mathcal{M}, \mathbb{P}\left(w_{0}, \ldots, w_{k}\right) \models_{S}^{3} \diamond \phi$ sse existe um mundo $w_{n+1} \in W$ tal que $w_{k} R w_{m+1} \mathrm{e}$ $\mathcal{M}, \mathbb{P}\left(w_{0}, \ldots, w_{k}, w_{m+1}\right) \models_{S}^{3} \phi$

FWG7. se $\neg \operatorname{Cond}\left(\mathbb{P}\left(w_{0}, \ldots, w_{k}\right)\right)$, ou $\mathcal{M}, \mathbb{P}\left(w_{0}, \ldots, w_{k}\right) \models_{S}^{3} \square \phi$ ou $\mathcal{M}, \mathbb{P}\left(w_{0}, \ldots, w_{k}\right) \nvdash_{S}^{3} \square \phi$

FWG8. se $\neg \operatorname{Cond}\left(\mathbb{P}\left(w_{0}, \ldots, w_{k}\right)\right)$, ou $\mathcal{M}, \mathbb{P}\left(w_{0}, \ldots, w_{k}\right) \models_{S}^{3} \diamond \phi$ ou $\mathcal{M}, \mathbb{P}\left(w_{0}, \ldots, w_{k}\right) \nvdash_{S}^{3} \diamond \phi$

FWG9. se $\phi \in S, \mathcal{M}, \mathbb{P}\left(w_{0}, \ldots, w_{k}\right) \models_{S}^{3} \neg \phi$ sse $\mathcal{M}, \mathbb{P}\left(w_{0}, \ldots, w_{k}\right) \nvdash_{S}^{3} \phi$

FWG10. não acontece simultaneamente que $\mathcal{M}, \mathbb{P}\left(w_{0}, \ldots, w_{k}\right) \nvdash_{S}^{3} \phi \mathrm{e}$ $\mathcal{M}, \mathbb{P}\left(w_{0}, \ldots, w_{k}\right) \not \nvdash_{S}^{3} \neg \phi$

Assim, a semântica tem por parâmetro os caminhos entre os mundos, e é por condições sobre esses caminhos que se determinam as condições de verdade sobre as necessitações e possibilitações. Suponha que a condição seja satisfeita. Então uma certa sentença da forma $\square \phi$ é verdadeira em um caminho apenas quando, para todos os caminhos "um passo adiante" desse caminho, $\phi$ for verdadeira. Um mundo pode ser pensado como um caminho de comprimento unitário. As condições de verdade FWE1-10 são casos particulares dessa semântica, considerando que $\operatorname{Cond}\left(\mathbb{P}\left(w_{0}, \ldots, w_{k}\right)\right)$ é $n-\left(\left|\mathbb{P}\left(w_{0}, \ldots, w_{k}\right)\right|-1\right)>0$.

Uma questão interessante que pode ser pensada sobre caminhos é a seguinte: dados dois mundos fixados $w$ e $t$, considere a coleção de todos os caminhos $\mathcal{D}_{w t}=\left\{\mathbb{P}\left(w, w_{0} \ldots, w_{k}, t\right): w_{i} \in W, 0 \leq i \leq m\right\}$ (se $m<0$, assume-se que não existem termos entre $w$ e $t$ ). Dessa coleção, é possível considerar o menor comprimento possível de caminhos, ou seja, $\iota(w, t)=\min \left\{\left|\mathbb{P}\left(w, w_{0} \ldots, w_{k}, t\right)\right|\right.$ : $\left.\mathbb{P}\left(w, w_{0} \ldots, w_{k}, t\right) \in \mathcal{D}_{w t}\right\}$. Para mundos $w$ e $t$ que não podem ser ligados por nenhum caminho, assume-se que $\iota(w, t)=\infty$; por fim, supõe-se que $\iota(w, w)=0$. Essa função $\iota$ satisfaz quase todas as propriedades de uma métrica, como será visto a seguir.

Seja $M$ um conjunto. Uma métrica em $M$ é uma função $d: M \times M \rightarrow \mathbb{R}$ que satisfaz os seguintes axiomas [Lim93]:

d1. $d(x, x)=0$, para todo $x \in M$ 
d2. Se $x \neq y$, então $d(x, y)>0$ para todo $x, y \in M$

d3. $d(x, y)=d(y, x)$ para todo $x, y \in M$

d4. $d(x, z) \leq d(x, y)+d(y, z)$ para todo $x, y, z \in M$

No caso em questão, $\iota$ é uma função $\iota: W \times W \rightarrow \mathbb{N} \cup\{\infty\}$. O axioma d1 é satisfeito por definição, isto é, $\iota(w, w)=0$ para todo $w \in W$. Se $t \neq w$, então há duas possibilidades. Na primeira, existe pelo menos um caminho $\mathbb{P}(w, \ldots, t)$ e, portanto, existe um mínimo; assim $0<\iota(w, t)<\infty$. Na segunda, não existe nenhum caminho entre $w$ e $t$, e nesse caso por definição $0<\iota(w, t)=\infty$. Em todos os casos, $\iota(w, t)>0$ se $w \neq t$, satisfazendo $\mathrm{d} 2$. O axioma $\mathrm{d} 3$ não é satisfeito porque não é possível garantir que, se $t$ é acessível a $w$, também $w$ é acessível a $t$, e muito menos que o menor caminho entre os dois mundos tenha o mesmo comprimento. Por fim, suponha que $\iota(w, t)=k_{1}$ e $\iota(t, v)=k_{2}$. Então existe um caminho de $w$ a $t$ de comprimento $k_{1}$ e outro de $t$ a $v$ de comprimento $k_{2}$. Justapondo esses caminhos, obtém-se um terceiro caminho que liga $w$ a $v$, e portanto $\iota(w, v) \leq k_{1}+k_{2}=\iota(w, t)+\iota(t, v)$, o que mostra que $\iota$ satisfaz d4.

Esses fatos fazem com que $\iota$ seja quase uma métrica em $W$, possuindo portanto várias propriedades interessantes. Intuitivamente, $\iota(w, t)$ é uma função que dá o menor número de passos introspectivos pelos quais é possível raciocinar sobre o mundo $t$ a partir de $w$. Quando $t=w$, não é necessária nenhuma introspeç̧ão, e quando não existe caminho de $w$ a $t$, são necessários infinitos passos. Uma questão interessante é como relacionar o conceito de $\iota$ com as possíveis condições $\operatorname{Cond}\left(\mathbb{P}\left(w_{0}, \ldots, w_{k}\right)\right)$. Pensando em $\iota$ como uma métrica, podem-se introduzir considerações topológicas sobre a semântica, o que permite utilizar todo o potencial dessa frutífera área da Matemática. Essas e outras questões podem ser analisadas em trabalhos futuros (ver Seção 8.2). 


\section{Capítulo 7}

\section{Lógica do Conhecimento de Finger-Wassermann}

O objetivo deste capítulo é mostrar que a semântica de Finger-Wassermann, apresentada na Seção 5.1, pode ser formalizada (da mesma forma que a semântica de Cadoli-Schaerf) em uma lógica modal do conhecimento. Em essência, os conceitos e teoremas são iguais àqueles de Cadoli e Schaerf apresentados na Seção 3.2 , bem como as demonstrações. Entretanto, por completude, isso será feito aqui. Como nos outros capítulos, $A$ representa o conjunto de átomos da linguagem.

A cada conjunto $S$ de proposições relevantes, associa-se um operador modal de conhecimento $\square_{S}^{3}$. Aqui, utiliza-se a mesma notação da Seção 3.2, mas não há risco de confusão porque fica subentendido que a semântica que se deseja emular é a de Finger-Wassermann. Sentenças da linguagem são construídas sobre o conjunto de literais $A^{\circ}$ utilizando os conectivos binários $\Lambda, \vee, \supset$ e os conectivos unários $\neg, \square \square_{S}^{3}$ e $\neg \square_{S}^{3}$. Para a Lógica do Conhecimento de CadoliSchaerf, as sentenças deveriam estar em MNNF. Aqui, a única restrição é de que o operador $\neg$ é aplicável somente a sentenças que não contenham operadores modais; o operador $\neg \square_{S}^{3}$, que aparentemente é uma combinação de $\neg$ com $\square_{S}^{3}$, terá uma semântica específica e por isso pode ser considerado como independente. Exemplos de sentenças dessa linguagem: $\square_{S}^{3}(p \vee \neg(q \supset r))$; $\neg \square{ }_{S}^{3}\left(p \wedge \square_{S}^{3} \neg \square_{S}^{3}(\neg(p \supset q))\right.$. Exemplos de sentenças que não são dessa linguagem: $\neg\left(p \vee \neg \square \square_{S}^{3} q\right), \square_{S}^{3}\left(p \supset \neg\left(q \wedge \neg \square_{S}^{3} r\right)\right)-$ em ambos os casos, há um operador $\neg$ que se aplica a uma subsentença que tem o operador modal $\neg \square_{S}^{3}$.

Definição 7.1 Um modelo Finger-Wassermann é uma tripla $\mathcal{M}=\langle$ Sit, $R, V\rangle$, em que Sit é um conjunto não vazio de situações, $R \subseteq$ Sit $\times$ Sit é uma relação de acessibilidade $\mathrm{e}:$ Sit $\rightarrow v_{S}^{3}$ é uma função que associa a cada situação uma valoração $S_{3}$ para algum $S \subseteq A$.

Nota-se que cada $s \in$ Sit pode ser associado, por $V$, a uma valoração $v_{S}^{3}$ para um $S$ diferente. Ou seja, se $s \neq t, V(s)=v_{S}^{3}$ e $V(t)=v_{S^{\prime}}^{3}$, então pode ocorrer 
$S \neq S^{\prime}$. O conjunto dos $t \in$ Sit tais que $V(s)$ é uma valoração $S_{3}$ é denotado por $S_{3}\left(\right.$ Sit). Observa-se também que, pelo Teorema 5.1, uma valoração $S_{3}$ é determinada não apenas por seu valor nos átomos $A$ como também no conjunto $\left\{\neg \phi \mid \phi \notin S\right.$ e $\left.v_{S}^{3}(\phi)=1\right\}$. Cada valoração $S_{3}$ associada a uma situação possui essa característica.

A semântica é dada pelas regras abaixo (recordando que uma sentença proposicional é uma sentença em que não aparecem operadores modais):

FWK1. $\mathcal{M}, s \models \phi$, quando $\phi$ for proposicional, sse $V(s)(\phi)=1^{1}$

FWK2. $\mathcal{M}, s \models \phi \vee \psi$ sse $\mathcal{M}, s \models \phi$ ou $\mathcal{M}, s \models \psi$

FWK3. $\mathcal{M}, s \models \phi \wedge \psi$ sse $\mathcal{M}, s \models \phi$ e $\mathcal{M}, s \models \psi$

FWK4. $\mathcal{M}, s \models \phi \supset \psi$ sse $\mathcal{M}, s \not \models \phi$ ou $\mathcal{M}, s \models \psi$

FWK5. $\mathcal{M}, s \models \square_{S}^{3} \phi$ sse para todo $t \in S_{3}($ Sit) tal que $s R t, \mathcal{M}, t \models \phi$ $\mathcal{M}, s \models \neg \square_{S}^{3} \phi$ sse existe $t \in S_{3}($ Sit $)$ tal que $s R t$ e $\mathcal{M}, t \not \models \phi$

Observa-se que, a princípio, não há nenhuma restrição sobre a relação de acessibilidade $R$; a associação do operador $\square_{S}^{3}$ à semântica depende tanto de $R$ como de $S_{3}$ (Sit). Denota-se por $\mathcal{W}($ Sit $)$ o conjunto de todas as situações $s \in$ Sit tais que $V(s)$ é uma valoração clássica, ou 2-interpretação. Essas situações podem ser chamadas de mundos possiveis. Uma sentença $\phi$ é válida, $\models \phi$, se para todo mundo possível $w \in \mathcal{W}($ Sit $)$ de todo modelo Finger-Wassermann $\mathcal{M}$, ocorrer $\mathcal{M}, w \models \phi$. Uma sentença $\phi$ é satisfativel se existir um mundo possível $w \in \mathcal{W}$ (Sit) e um modelo Finger-Wassermann $\mathcal{M}$ tais que $\mathcal{M}, w \models \phi$, e é insatisfativel se isso não ocorrer.

Essas noções generalizam as semântica CS1-5 de Cadoli-Schaerf dada na Seção 3.2) porque quando se restringe a sentenças em MNNF, recupera-se aquela semântica.

A ligação entre $\square_{S}^{3}$ e $\models_{S}^{3}$ da Seção 5.1 é dada pelo

Teorema 7.2 (Validade modal e $S_{3}$-inferência) Sejam $\phi$ e $\psi$ duas sentenças proposicionais. Então $\models \square_{S}^{3} \phi \supset \square_{S}^{3} \psi$ sse $\square_{S}^{3} \phi \wedge \neg \square_{S}^{3} \psi$ for insatisfativel classicamente sse $\phi \models_{S}^{3} \psi$.

Dem: A prova é idêntica à do Teorema 3.7 (encontrada em [SC95]), mas será dada aqui por se tratar de outra semântica. É necessário mostrar três implicações:

1. $\square_{S}^{3} \phi \wedge \neg \square \square_{S}^{3} \psi$ ser insatisfatível implica que $\models \square_{S}^{3} \phi \supset \square_{S}^{3} \psi$

2. $\models \square_{S}^{3} \phi \supset \square_{S}^{3} \psi$ implica que $\phi \models_{S}^{3} \psi$

3. $\phi \models_{S}^{3} \psi$ implica que $\square_{S}^{3} \phi \wedge \neg \square \square_{S}^{3} \psi$ é insatisfatível

${ }^{1}$ Ou seja, o valor verdade de $\phi$, calculado utilizando as Regras FW1-5 dadas na Seção 5.1, é igual a 1 . 
Prova de 1: Assuma que $\square_{S}^{3} \phi \wedge \neg \square_{S}^{3} \psi$ seja insatisfatível e que $\not \models \square_{S}^{3} \phi \supset \square_{S}^{3} \psi$. Então existem um modelo $\mathcal{M}=\langle$ Sit, $R, V\rangle$ e um mundo possível $w \in$ $\mathcal{W}($ Sit) tais que a seguinte seqüência de fatos é verdadeira (cada elemento da seqüência é implicado pelo anterior):

1. $\mathcal{M}, w \not \models \square_{S}^{3} \phi \supset \square_{S}^{3} \psi$

2. $\mathcal{M}, w \models \square_{S}^{3} \phi$ e $\mathcal{M}, w \not \models \square_{S}^{3} \psi$

3. $\mathcal{M}, w \models \square_{S}^{3} \phi$ e existe $t \in S_{3}($ Sit $)$ tal que $w R t$ e $\mathcal{M}, t \not \models \psi$

4. $\mathcal{M}, w \models \square_{S}^{3} \phi$ e $\mathcal{M}, w \models \neg \square_{S}^{3} \psi$

5. $\mathcal{M}, w \vDash \square_{S}^{3} \phi \wedge \neg \square_{S}^{3} \psi$

O último fato da seqüência mostra que $\square_{S}^{3} \phi \wedge \neg \square_{S}^{3} \psi$ é satisfatível, contradizendo a hipótese.

Prova de 2: Assuma que $\models \square_{S}^{3} \phi \supset \square_{S}^{3} \psi$ mas que não ocorra $\phi \models_{S}^{3} \psi$ (ou seja, $\left.\phi \not \nvdash_{S}^{3} \psi\right)$. Então existe uma valoração ${v_{S}^{\prime}}_{S}^{3}$ tal que ${v_{S}^{\prime}}_{S}^{3}(\phi)=1$ mas ${v^{\prime}}_{S}^{\prime 3}(\psi)=0$. Seja $\mathcal{M}=\langle$ Sit $, R, V\rangle$ em que $\operatorname{Sit}=\{w, s\}, R=\{(w, s)\}$, $V(w)=v$, em que $v$ é uma valoração clássica qualquer, e $V(s)={v^{\prime}}_{S}^{3}$. Então $\mathcal{W}($ Sit $)=\{w\}$. Tem-se que $\mathcal{M}, s \models \phi$ e $\mathcal{M}, s \not \models \psi$. Como $s$ é a única situação em Sit acessível a $w$, tem-se também que $\mathcal{M}, w \models \square_{S}^{3} \phi$ e $\mathcal{M}, w \forall \square_{S}^{3} \psi$, o que significa que $\mathcal{M}, w \not \models \square_{S}^{3} \phi \supset \square_{S}^{3} \psi$, contrário à hipótese.

Prova de 3: Assuma que $\phi \models_{S}^{3} \psi$ e que $\square_{S}^{3} \phi \wedge \neg \square_{S}^{3} \psi$ é satisfatível. Então existem um modelo $\mathcal{M}=\langle$ Sit $, R, V\rangle$ e um mundo possível $w \in \mathcal{W}($ Sit $)$ tais que a seguinte seqüência de fatos é verdadeira (cada elemento da seqüência é implicado pelo anterior):

1. $\mathcal{M}, w \models \square{ }_{S}^{3} \phi \wedge \neg \square \square_{S}^{3} \psi$

2. $\mathcal{M}, w \models \square_{S}^{3} \phi$ e $\mathcal{M}, w \not \models \square_{S}^{3} \psi$

3. para todo $t \in S_{3}($ Sit $)$ tal que $s R t, \mathcal{M}, t \models \phi$ e existe $t \in S_{3}($ Sit $)$ tal que $w R t$ e $\mathcal{M}, t \not \neq \psi$

4. existe $t \in S_{3}($ Sit $)$ tal que $w R t \mathrm{e}$, simultaneamente, $\mathcal{M}, t \models \phi \mathrm{e}$ $\mathcal{M}, t \forall \neg \neg \psi$

Como $V(t)$ é uma valoração $S_{3}$, que pode ser denotada ${v^{\prime}}_{S}^{3}$, tem-se que ${v^{\prime}}_{S}^{3}(\phi)=1$ e ${v^{\prime}}_{S}^{3}(\psi)=0$, que implica que $\phi \not \nvdash_{S}^{3} \psi$, contradição.

Como é de se esperar, vale a monotonicidade:

Teorema 7.3 (Monotonicidade para $\square_{S}^{3}$ ) Seja $S \subseteq S^{\prime} \subseteq A$. Então, tem-se $q u e \models \square_{S}^{3} \phi \supset \square_{S^{\prime}}^{3} \psi$.

Dem: Assuma que $\not \models \square_{S}^{3} \phi \supset \square_{S^{\prime}}^{3} \phi$. Então existem um modelo $\mathcal{M}=$ $\langle$ Sit, $R, V\rangle$ e um mundo possível $w \in \mathcal{W}($ Sit $)$ tais que a seguinte seqüência de fatos é verdadeira (cada elemento da seqüência é implicado pelo anterior): 
1. $\mathcal{M}, w \not \models \square_{S}^{3} \phi \supset \square_{S^{\prime}}^{3} \phi$

2. $\mathcal{M}, w \models \square_{S}^{3} \phi$ e $\mathcal{M}, w \not \models \square_{S^{\prime}}^{3} \phi$

3. para todo $t \in S_{3}\left(\right.$ Sit) tal que $s R t, \mathcal{M}, t \models \phi$ e existe $t \in S_{3}^{\prime}($ Sit $)$ tal que $s R t$ e $\mathcal{M}, t \not \neq \phi$

Como $\phi$ é proposicional, tem-se que $V(t)(\phi)=0$; e $V(t)$ é uma valoração $S_{3}^{\prime}$. Portanto, existe uma valoração $S_{3}^{\prime}$, que será denotada por ${v^{\prime}}_{S^{\prime}}^{\prime 3}$, tal que ${v^{\prime}}_{S^{\prime}}^{\prime 3}(\phi)=$ 0 , mas toda valoração $S_{3}$ satisfaz $\phi$. Como mostrado no Teorema 5.4, quando $S \subseteq S^{\prime}$, toda valoração $S_{3}^{\prime}$ é uma valoração $S_{3}$. Portanto, $S_{3}($ Sit $) \subseteq S_{3}^{\prime}($ Sit $)$. Assim, $v_{S^{\prime}}^{\prime 3}$ é uma valoração $S_{3}$ e deveria satisfazer $\phi$, o que não ocorre: contradição.

Assim, como na Seção 3.2, e pelas mesmas razões (a demonstração é a mesma), vale o

Teorema $7.4 \vDash \square_{A}^{3} \phi \supset \phi$.

Uma questão interessante a ser estudada é o que ocorre aos axiomas e regras de inferência caracterizadores do sistema modal S5 quando se substitui o operador $\square$ por $\square_{S}^{3}$. Lembra-se que S5 tem por regra de inferência modal a

Regra da Necessitação: se $\phi$ é um teorema, então $\square \phi$ também é um teorema e os axiomas:

K. $\square(p \supset q) \supset(\square p \supset \square q)$

T. $\square p \supset p$

4. $\square p \supset \square \square p$

5. $\neg \square p \supset \square \neg \square p$

Também observa-se que a relação de acessibilidade $R$ de todos os modelos para S5 pode ser considerada reflexiva, transitiva e euclidiana.

$\mathrm{Na}$ Seção 3.2, demonstrou-se que todos os princípios à exceção de $K$ eram válidos. Poderia parecer que o mesmo ocorreria neste caso, em que a semântica em questão é Finger-Wassermann. Mas não é o que ocorre. Aqui, todos os princípios são válidos e a regra da necessitação falha. A seguir, isso será mostrado e também será discutido o porquê do comportamento diferente daquele da Seção 3.2.

O contra-exemplo para a regra da necessitação é o seguinte. Seja $\mathcal{M}=$ $\langle$ Sit, $R, V\rangle$, de forma que:

- $\phi=\neg(p \wedge \neg p)$

- $S=\emptyset$, Sit $=\left\{s_{1}, s_{2}\right\}, R=$ Sit $\times$ Sit

- $V\left(s_{1}\right)(p)=V\left(s_{2}\right)(p)=V\left(s_{2}\right)(\neg p)=1$ e $V\left(s_{1}\right)(\neg p)=V\left(s_{2}\right)(\neg(p \wedge \neg p))=$ 
A função $V$ é, para $s_{1}$, uma valoração clássica (e, dessa maneira, uma valoração $\left.S_{3}\right)$ e, para $s_{2}$, uma valoração $S_{3}$. Nota-se que, pelo Teorema 5.1 , o valor de $V\left(s_{2}\right)(\neg(p \wedge \neg p))$ precisa ser determinado, uma vez que, por FW1, $V\left(s_{2}\right)((p \wedge$ $\neg p))=1$ e $p \notin S$. Por outro lado, como $V\left(s_{1}\right)$ é clássica, $V\left(s_{1}\right)(\neg(p \wedge \neg p))=1$, uma vez que $V\left(s_{1}\right)(p \wedge \neg p)=0$. Tem-se que $S_{3}($ Sit $)=\left\{s_{1}, s_{2}\right\}$. Além disso, $\mathcal{W}($ Sit $)=\left\{s_{1}\right\}$. Como $s_{1}$ é o único mundo possível em relação ao conjunto Sit, e para $s_{1}$ a sentença $p \vee \neg p$ é verdadeira, então $\mathcal{M} \models \phi$. Entretanto, $\mathcal{M} \not \square_{S}^{3} \phi$, uma vez que $\mathcal{M}, s_{2} \not \models \neg(p \wedge \neg p)$ e, $s_{1}$ acessando $s_{2}, \mathcal{M}, s_{1} \not \models \square_{S}^{3} \neg(p \wedge \neg p)$.

O comportamento diferente do operador $\square_{S}^{3}$, com relação ao do operador homônimo da Seção 3.2, pode ser explicado. Naquela seção, era assumido que as sentenças estavam em MNNF, e a prova da validade da regra da necessitação dependia disso. Aqui, com uma liberdade maior para a estrutura das sentenças, certos fatos já não podem mais ser assumidos. Portanto, intuitivamente, o fato de que $\phi$ é verdade não implica que isso seja conhecido por um agente que utiliza um raciocínio aproximado. De certa forma, é como se nem todas as verdades possam ser conhecidas por esse agente, o que é filosoficamente mais aceitável que o resultado da Seção 3.2 .

O princípio $T$ valia porque toda valoração clássica era também uma valoração $S_{3}$ e também porque a relação de acessibilidade $R$ era reflexiva, e portanto continua valendo aqui. O princípio 4 saía da hipótese de que $R$ era transitiva, e o princípio 5 do fato que a relação, sendo transitiva e euclidiana, era também simétrica. Assim, ambos continuam valendo também.

O mesmo contra-exemplo oferecido para refutar $K$ pode continuar sendo usado (afinal, a semântica de Finger-Wassermann contém Cadoli-Schaerf como caso particular), só que isso apenas demonstraria que $\forall \neg \neg \square_{S}^{3}(\neg p \vee q) \vee\left(\neg \square \square_{S}^{3} \vee\right.$ $\square_{S}^{3} q$ ). Afinal, para Cadoli-Schaerf $p \supset q$ é apenas uma abreviação para $\neg p \vee q$. Só que, para Finger-Wassermann, $p \supset q$ não é equivalente a $\neg p \vee q$. Tal equivalência só acontece se $p$ e $q$ estiverem no conjunto de proposições relevantes $S$. Na Seção 5.1 , mostrou-se que as duas formas de modus ponens (primeira forma: de $\phi \mathrm{e}$ $\phi \supset \psi$ pode-se inferir $q$; segunda forma: de $\phi \mathrm{e} \neg \phi \vee \psi$ pode-se inferir $\psi$ ) não são equivalentes. A primeira forma sempre vale, mas a segunda não. $\mathrm{E}$ é por essa razão que o princípio $K$, formulado com o operador $\supset$, vale para FingerWassermann: esse operador se comporta classicamente. De fato, suponha que, para um modelo $\mathcal{M}=\langle$ Sit $, R, V\rangle$ e um mundo $w$ desse modelo, ocorra $\mathcal{M}, w \models$ $\square_{S}^{3}(p \supset q)$ e $\mathcal{M}, w \models \square_{S}^{3} p$. Então, para toda situação $s \in S_{3}($ Sit $)$ tal que $w R s$, ocorre simultaneamente $\mathcal{M}, s \models p \supset q$ e $\mathcal{M}, s \models p$. Em suma, $V(s)(p \supset q)=1 \mathrm{e}$ $V(s)(p)=1$. Como $V(s)$ é uma valoração $S_{3}$, pela condição FW3 da Seção 5.1, tem-se necessariamente que $V(s)(q)=1$, o que implica que $\mathcal{M}, s \models q$. Como isso vale para todo $s \in S_{3}($ Sit $)$ tal que $w R s$, conclui-se que $\mathcal{M}, w \models \square_{S}^{3} q$, o que demonstra $K$.

Esse último resultado pode ser interpretado da seguinte forma: a onisciência lógica, englobada por $K$, vale se, nesse princípio, for utilizado o operador $\supset$, $\mathrm{e}$ não vale se forem utilizados $\neg \mathrm{e} \vee$ no lugar de $\supset$. Assim, segundo os sinais da linguagem que se utiliza, há ou não onisciência. Isso é filosoficamente insatisfatório. Para um agente dotado de recursos finitos, a onisciência jamais poderia valer. A única forma de resolver a questão é controlar também o comportamento do ope- 
rador $\supset$, como foi feito na semântica generalizada $S_{e}$ de Finger-Wassermann, explicada no final da Seção 5.2. A questão pode ser analisada em trabalhos futuros. 


\section{Capítulo 8}

\section{Conclusões}

Neste capítulo final, é feito um panorama do trabalho, analisando-se e comparando os diferentes métodos de aproximação. Depois, sugestões para trabalhos futuros são apresentadas.

\subsection{Análise}

Em seu artigo "Anytime approximate modal reasoning" [Mas98b], Fabio Massacci observa a existência de duas fontes independentes de complexidade nas lógicas proposicionais modais: onisciência lógica e introspecção ilimitada.

No Capítulo 1, foi discutido o significado da onisciência lógica - que é representada na teoria de Hintikka pelo princípio modal $K-\mathrm{e}$ o trabalho de Hector Levesque, que procura controlar essa onisciência. Levesque utiliza uma linguagem bimodal, generalizando a semântica de mundos possíveis de Hintikka para uma de situações, modelando dois tipos de crenças: a crença implícita e explícita. A crença implícita possui as mesmas propriedades do operador de crença de Hintikka, mas a crença explícita passa por cima de eventuais contradições, além de não se aplicar necessariamente a todas as tautologias e equivalências lógicas. Ocorre que as propriedades computacionais da crença explícita são mais atraentes. Em [Lev84], é provado que a inferência de uma crença implícita por outra explícita é um problema co-NP-completo ("muito difícil"), mas que a inferência de uma crença explícita por outra explícita pode ser realizada em tempo polinomial ("relativamente fácil"). Com uma restrição posterior de Levesque em sua semântica (apresentada no artigo [Lev89]), a crença explícita pode ser interpretada, sem operadores modais, como sentenças em NNF em que há uma generalização da semântica de dois valores clássica. Para cada par de literais $p$ e $\neg p$, há três possibilidades: ou $p$ é levado em 1 e $\neg p$ em 0 , ou $p$ é levado em 0 e $\neg p$ em 1 , ou $p$ e $\neg p$ são ambos levados em 1 . Essa valoração é chamada de 3-interpretação, e mostra-se (Teorema da Correção) que, se uma inferência de uma sentença por outra é realizável por essa 3-interpretação, então ela é realizável classicamente também. A abordagem de Levesque não permite 
uma incrementação posterior: se um problema de inferência entre sentenças puder ser resolvido com a 3-interpretação, que via de regra é mais fácil, então ele é automaticamente solúvel classicamente; se, entretando, o problema não puder ser resolvido pela 3-interpretação, é preciso voltar ao problema original para tentar resolvê-lo da maneira usual.

Em outra seção do Capítulo 1, é apresentado um procedimento computacional que se propõe também a simplificar o problema da inferência lógica entre sentenças. Esse procedimento foi chamado compilação de conhecimento por seus autores Bart Selman e Henry Kautz. Esse método toma uma sentença e tenta aproximá-la, da melhor forma possível, por teorias Horn, que possui propriedades computacionais mais atraentes. Aproximar sentenças, aqui, significa obter uma teoria Horn que consiga realizar o maior número possível das inferências realizáveis pela sentença original. Há, como se pode imaginar, dois tipos de aproximações: a por teorias Horn que façam menos inferências que as sentenças originais, e a por teorias Horn que façam mais inferências. As primeiras são chamadas de aproximações por baixo, e as últimas aproximações por cima. $\mathrm{O}$ fato de que se todas as inferências que podem ser feitas pelas aproximações por baixo podem ser feitas pela sentença original é referido como correção da aproximação por baixo. $\mathrm{O}$ fato de que todas as inferências que podem ser feitas pela sentença por baixo podem ser feitas pelas aproximações por cima é referido como completude das aproximações por cima. O procedimento de Selman e Kautz também não pode ser incrementado posteriormente.

As idéias de Levesque e Selman e Kautz serviram de inspiração para um método de aproximações de lógicas desenvolvido por Marco Cadoli e Marco Schaerf, apresentado no Capítulo 3 . Buscando a incrementabilidade que faltava nos procedimentos anteriores, Cadoli e Schaerf selecionam um subconjunto de proposições relevantes $S$ (ao qual se associa um subconjunto $S^{\circ}$ dos literais) contido no conjunto de todos os átomos. Para pares de literais fora de $S^{\circ}$, o comportamento é semelhante ao da 3-interpretação de Levesque, mas literais em $S^{\circ}$ necessariamente se comportam classicamente. Tais valorações do conjunto de todos os átomos são chamadas de $S_{3}$-interpretações. A linguagem é restrita a sentenças em NNF. Cadoli e Schaerf mostram a propriedade da monotonicidade, que generaliza o Teorema da Correção de Levesque: se $S \subseteq S^{\prime}$ e a inferência de uma sentença por outra puder ser feita pela $S_{3}$-interpretação, então ela pode ser feita pela $S_{3}^{\prime}$-interpretação. Via de regra, quanto menor o conjunto $S$, mais fácil a resolução de problemas de inferência. Quando $S=\emptyset$, volta-se à 3-interpretação de Levesque, e quando $S$ é o conjunto de todos os átomos retorna-se à interpretação clássica de dois valores. Se o problema não pode ser resolvido com o conjunto $S$, aumenta-se o conjunto para $S^{\prime}$, e a monotonicidade implica que o que já podia ser resolvido com $S$ continua valendo para $S^{\prime}-$ e assim é solucionada a questão da incrementabilidade. Para aproximações por cima, Cadoli e Schaerf sugerem uma semântica de aproximação $S_{1}$. Nessa semântica, fora de $S$ o comportamento dos literais é clássico, mas literais dentro de $S$ são ambos levados para o valor 0 - essa é a definição de $S_{1}$-interpretação. Quando $S$ é o conjunto de todos os átomos, retorna-se à interpretação clássica. A monotonicidade, nesse caso, significa que se $S \subseteq S^{\prime}$ então 
toda inferência entre sentenças realizada pela $S_{1}^{\prime}$-interpretação pode ser feita pela $S_{1}$-interpretação, ou, numa formulação mais utilizada, toda refutação de sentenças que pode ser feita pela $S_{1}$-interpretação também pode ser realizada também pela $S_{1}^{\prime}$-interpretação, preservando a completude das aproximações por cima.

Esses fatos podem ser analisados e na formalização que Cadoli e Schaerf fizeram de suas semânticas em uma lógica do conhecimento. Quando isso é feito, incorporando-se um para de famílias de operadores $\square_{S}^{3}$ e $\square_{S}^{1}$ para cada conjunto $S$ possível, torna-se possível demonstrar que as $S_{3}$-aproximações de CadoliSchaerf resolvem o problema da onisciência na Lógica Clássica para fórmulas em NNF, na medida em que um agente pensante não é mais capaz de determinar todas as consequiências lógicas de suas crenças, mas apenas aquelas mais diretamente relacionadas com as mesmas. Com mais tempo e recursos, ele é capaz de inferir mais, o que corresponde a aumentar o conjunto das proposições relevantes. Mostra-se também que as $S_{1}$-aproximações mantém a onisciência da Lógica Clássica (o que era de se esperar, uma vez que as lógicas que aproximam por cima mantém todos os teoremas da Lógica Clássica), mas não a correção de todas as inferências - certas inferências incorretas também podem ser feitas.

Cadoli e Schaerf trabalharam uma extensão de seu procedimento para sentenças modais em MNNF. No seu método, eles determinam uma semântica que controla a capacidade introspectiva do problema, no que chamaram de $\left\langle S_{3}, i\right\rangle$ aproximações.

Fabio Massacci desenvolveu, em sua tese de doutorado [Mas98a], um procedimento que estende Cadoli-Schaerf para sentenças quaisquer mas que utiliza constantemente sentenças assinaladas. Sua análise é detalhada, descrevendo inclusive a complexidade das lógicas obtidas.

Adicionalmente, ele trabalhou um procedimento de aproximações de lógicas modais que regula tanto o conjunto de proposições relevantes como o conjunto de mundos interessantes. Esse procedimento permite um controle fino sobre o poder de introspecção dos agentes modais.

Em todos os casos, Massacci oferece uma teoria da prova, sugere um algoritmo de incrementabilidade e mostra a correção e completude dessa teoria com relação à semântica. Como sua pesquisa busca primariamente um procedimento computacional eficiente, ele não formalizou suas semânticas aproximadas em uma lógica do conhecimento.

O trabalho de Massacci foi discutido no capítulo 4.

O trabalho de Marcelo Finger e Renata Wassermann, estudado no Capítulo 5 , estende o conceito de aproximações para sentenças quaisquer - e não assinaladas - da Lógica Clássica. Além disso, fazem uma teoria da prova por meio de uma adaptação dos KE-tableaux de Mondadori e d'Agostino, que foi chamada de $\mathrm{KE} S_{3}$-tableau. Esse tableau sugere um algoritmo incremental para o conjunto de proposições relevantes.

Uma possível extensão para lógicas modais do método de Finger-Wassermann foi realizada no Capítulo 6. Nessa extensão, há dois parâmetros que controlam a verdade de sentenças: o conjunto de proposições relevantes $S$ e o limite máximo de introspecção $n$. Dentro do conjunto de proposições relevan- 
tes, a linguagem se comporta classicamente, desde que o número de interações modais não supere $n$. Fora de $S$, toleram-se contradições. Além do limite de introspecção modal, tudo é possível e nada é necessário. O procedimento apresenta uma semântica e uma teoria da prova no contexto dos SST de Massacci [Mas00], que foi chamada de $\left\langle S_{3}, n\right\rangle$-SST. Para esses tableaux, uma nova regra de fechamento precisa ser introduzida. Em outra seção, resolve-se o problema da criação de uma nova regra de fechamento dos tableaux, necessária no caso dos $\left\langle S_{3}, n\right\rangle$-SST para a prova da completude, por meio de uma semântica que permite a escolha arbitrária da verdade das possibilitações e necessitações de proposições em mundos além do limite máximo de introspecção $n$. O procedimento é caracterizado por uma grande simplicidade, com um algoritmo de fácil implementação; o algoritmo admite incrementações do conjunto $S$ de proposições relevantes e do limite máximo de introspeç̧ão $n$. Os parâmetros permitem um controle fino sobre as demonstrações das sentenças. Na seção final do capítulo sugere-se uma semântica generalizada; essa semântica parece permitir maior controle sobre as demontrações, com condições mais gerais que um simples limite máximo para a capacidade introspectiva do sistema.

No Capítulo 7, formaliza-se a semântica de Finger-Wassermann em uma lógica modal do conhecimento. Assim como foi feito na Seção 3.2, é introduzida uma família de operadores $\square_{S}^{3}$ para cada subconjunto de proposições relevantes $S$. A análise das propriedades da linguagem obtida mostra que a falha da onisciência lógica ocorre apenas quando se formula o princípio $K$ com os operadores $\neg$ e $\vee$. O princípio $K$ formulado com $\supset$ continua valendo, o que mostra que há diferentes conceitos - e portanto diferentes graus - de onisciência, dependendo dos símbolos utilizados.

\subsection{Trabalhos futuros}

Há várias linhas de pesquisa que surgem a partir das considerações deste trabalho. A seguir, são citadas algumas.

1. Uma questão fundamental é o da complexidade da família de lógicas $\left\langle S_{3}, n\right\rangle$. O problema de provabilidade de sentenças, para grande parte dos sistemas de Lógica Modal, é PSPACE-completo [Lad77]. Em Lógica Clássica, o problema da provabilidade de uma sentença é NP-completo. Em [Fin04], Marcelo Finger apresenta uma família de lógicas que aproximam inferência clássica, de forma que cada passo da aproximação pode ser decidido em tempo polinomial, inspirando-se num trabalho já feito para lógica clausal por Dalal [Dal96a, Dal96b]. Sabe-se que todo problema NP-completo é PSPACE-completo, embora não se saiba se a recíproca é verdadeira ou falsa. Será que as lógicas $\left\langle S_{3}, n\right\rangle$ dão origem a problemas tratáveis polinomialmente? Nesse caso, como elas aproximam um problema PSPACE-completo, que tipo de luz elas trariam sobre a questão se PSPACE $\subseteq$ NP?

2. Na Seção 6.2, observou-se que a aplicação da regra (bey) pode diminuir 
consideravelmente o tamanho das provas, mas essas provas obtidas não são aceitáveis classicamente. A Figura 6.4 mostra que são precisos pelo menos dois passos introspectivos para demonstrar certo teorema de S5 sem o uso de (bey), e uma simples adaptação desse exemplo mostra que é possível encontrar teoremas de S5 que necessitam de no mínimo $n$ passos introspectivos para cada $n$ a fim de que a prova obtida não utilize (bey) e seja clássica. Ou seja, a seleção de um limite máximo de introspeç̧ão limita a quantidade de teoremas demonstráveis classicamente.

3. Um problema interessante que sai do estudo da Seção 6.3 é o seguinte. Observou-se naquela seção que, em alguns sistemas de lógicas modais, certos princípios que são considerados axiomas nos sistemas de Hilbert são demonstráveis por SST, podendo-se inclusive determinar o número de passos introspectivos para que a demonstração seja efetivada. A demonstração de todos os axiomas de um sistema modal após a seleção de um determinado limite máximo de introspeç̧ão não implica que todos os teoremas desse sistema também sejam demonstráveis. Ou seja, a seleção de um limite máximo de introspecção limita a quantidade de teoremas demonstráveis. A análise desse fenômeno, bem como o observado no item anterior, pode ser realizada em trabalhos futuros.

4. O desenvolvimento da teoria por trás da semântica generalizada da Seção 6.4 também pode fornecer resultados frutíferos. Um aspecto que o autor gostaria de ter incorporado na semântica de aproximações modais é a escolha de um subconjunto de mundos possíveis interessantes, assim como fez Massacci. Isso poderia ser feito, bem como a determinação de uma distância (utilizando a função $\iota$ definida na Seção 6.4) dentro da qual todos os mundos seriam interessantes, mas fora da qual nada o seria. A aplicação de ferramentas topológicas se apresenta naturalmente.

5. Uma linha natural de pesquisa é a extensão do trabalho para lógicas multimodais, em particular para lógicas temporais (como CTL, LTL, CTL ${ }^{*}$ ), conforme apresentadas, por exemplo, em [Bur84]. Para isso, seria necessário o estudo de SST para essas lógicas, trabalho que ainda precisa ser realizado.

6. Considere o resultado obtido no Capítulo 7, aonde se mostrou que o princípio $K$ vale quando formulado com o operador $\supset$ (ou seja, ( $\square p \supset$ q) $\supset(\square p \supset \square q)$ ) mas não vale quando formulado com $\neg$ e $\vee$ (ou seja, $\neg(\neg \square p \vee \square q) \vee(\neg \square p \vee \square q))$. Um estudo interessante é uma possível alteração da regra para o operador $\supset$, de forma que o princípio $K$ formulado com o mesmo falhe, quando a semântica é analisada à luz de uma lógica modal do conhecimento. 


\section{Referências Bibliográficas}

[AB75] Alan Ross Anderson and Nuel D. Belnap. Entailment, the Logic of Relevance and Necessity. Princeton University Press, 1975.

[Bel77] Nuel Belnap. A useful four-valued logic. In G. Epstein and J. Dunn, editors, Modern Uses of Multiple-Valued Logic, pages 8-37. D. Reidel Publishing Company, 1977.

[Boo54] George Boole. An Investigation of the Laws of Thought. Macmillan, Cambridge; London, 1854. Reimpressa pela Dover Publications a partir de 1954.

[Bur84] J. P. Burgess. Basic Tense Logic. In D. Gabbay and F. Guenthner, editors, Handbook of Philosophical Logic, volume II, pages 89-133. D. Reidel Publishing Company, 1984.

[Che80] B. F. Chellas. Modal Logic - an Introduction. Cambridge University Press, 1980.

[CPW00] Samir Chopra, Rohit Parikh, and Renata Wassermann. Approximate belief revision. In Proceedings of Workshop on Language, Logic and Information (WoLLIC), 2000.

[D'A90] Marcello D'Agostino. Investigations into the complexity of some propositional calculi. $\mathrm{PhD}$ thesis, Oxford University Computing Laboratory, November 1990. Publicada como PRG Technical Monographs 88.

[D'A92] Marcello D'Agostino. Are tableaux an improvement on truth-tables? - Cut-free proofs and bivalence. Journal of Logic, Language and Information, 1:235-252, 1992.

[D'A99] Marcello D'Agostino. Tableau methods for classical propositional logic. In Marcello D'Agostino, Dov Gabbay, Rainer Haehnle, and Joachim Posegga, editors, Handbook of Tableau Methods, pages 45124. Kluwer, 1999.

[Dal96a] Mukesh Dalal. Anytime families of tractable propositional reasoners. In International Symposium of Artificial Intelligence and Mathematics AI/MATH-96, pages 42-45, 1996. 
[Dal96b] Mukesh Dalal. Semantics of an anytime family of reasoners. In 12th European Conference on Artificial Intelligence, pages 360-364, 1996.

[Fin04] Marcelo Finger. Towards polynomial approximations of full propositional logic. Technical Report RT-2004-04, Department of Computer Science, IME/USP, 2004. Available for download at www. ime.usp.br/ mfinger/publications.

[Fit83] Melvin Fitting. Proof Methods for Modal and Intuitionistic Logics. D. Reidel Publishing Company, 1983.

[FW01] Marcelo Finger and Renata Wassermann. Tableaux for approximate reasoning. In Leopoldo Bertossi and Jan Chomicki, editors, IJCAI2001 Workshop on Inconsistency in Data and Knowledge, pages 7179, Seattle, August 6-10 2001.

[FW02] Marcelo Finger and Renata Wassermann. Logics for approximate reasoning: Approximating classical logic "from above". In Brazilian Symposium on Artificial Intelligence, pages 21-30, 2002. Lecture Notes in Artificial Intelligence, 2002. v.2507.

[FW04] Marcelo Finger and Renata Wassermann. Approximate and limited reasoning: Semantics, proof theory, expressivity and control. Journal of Logic And Computation, 14(2):179-204, 2004.

[Gen69] Gerhard Gentzen. Investigations into logical deduction. In M. Szabo, editor, The Collected Papers of G. Gentzen, pages 68-131. NorthHolland Publishing Company, 1969. Tradução inglesa de Untersuchungen über das logische Schließen, publicado em 1935.

[Gor99] Rajeev Goré. Tableau methods for modal and temporal logic. In Marcello D'Agostino, Dov Gabbay, Rainer Haehnle, and Joachim Posegga, editors, Handbook of Tableau Methods, pages 297-396. Kluwer, 1999.

[HA50] David Hilbert and Wilhelm Ackermann. Principles of Mathematical Logic. Chelsea Publishing Company, 1950. Tradução inglesa da segunda edição de Grundzüge der teoretischen logik, publicada em 1938; reimpressa pela American Mathematical Society em 1999.

[HC96] G. E. Hughes and M. J. Cresswell. A New Introduction to Modal Logic. Routledge, London and New York, 1996. Reimpresso em 2003.

[Hen49] Leon Henkin. The completeness of the first-order functional calculus. Journal of Symbolic Logic, 14:159-166, 1949.

[Hin62] Jaako Hintikka. Knowledge and Belief: An Introduction to the Logic of the Two Notions. Cornell University Press, 1962. 
[Hin75] Jaako Hintikka. Impossible worlds vindicated. Journal of Philosophical Logic, 4:475-484, 1975.

[Kle52] Stephen Cole Kleene. Introduction to Metamathematics, volume I of Bibliotheca Mathematica. North-Holland Publishing Company and Wolters-Noordhoff Publishing, 1952. Reimpresso em 1971.

[Lad77] Richard Ladner. The computational complexity of provability in systems of modal propositional logic. SIAM Journal of Computing, 6:467-480, 1977.

[Lev84] Hector Levesque. A logic of implicit and explicit belief. In Proceedings of $A A A I-84,1984$.

[Lev89] Hector Levesque. A knowledge-level account of abduction. In IJCAI89-Proceedings of the Eleventh International Joint Conference on Artificial Intelligence, pages 1061-1067, 1989.

[Lim93] Elon Lages Lima. Espaços Métricos. Projeto Euclides. IMPA - Instituto de Matemática Pura e Aplicada, Rio de Janeiro, third edition, 1993. Reimpresso em 2003.

[Łuka63] Jan Lukaziewicz. Elements of Mathematical Logic. Pergamon Press and PWN-Polish Scientific Publishers, 1963. Tradução inglesa da segunda edição de Elementy logiki matematycznej, publicada em 1958.

[Mas98a] F. Massacci. Efficient Approximate Deduction and an Application to Computer Security. PhD thesis, Universitá di Roma "La Sapienza", Dipartimento di Informatica e Sistemistica, June 1998.

[Mas98b] Fabio Massacci. Anytime approximate modal reasoning. In Jack Mostow and Charles Rich, editors, AAAI-98, pages 274-279. AAAIP, 1998.

[Mas98c] Fabio Massacci. Single step tableaux for modal logics: methodology, computations, algorithms. Technical Report TR-04, Dipartimento Informatica e Sistemistica, Univ. di Roma "La Sapienza", 1998. It extends article [Mas00] with a result on the impossibility of capitalizing validity information from one modal logic to another modal logic.

[Mas00] Fabio Massacci. Single step tableaux for modal logics: methodology, computations, algorithms. JAR, 24(3):319-364, 2000. The corresponding technical report [Mas98c] has an additional section with a result on the impossibility of capitalizing validity information from one modal logic to another modal logic.

[Mon88a] Marco Mondadori. Classical analytical deduction. In Annali dell'Università de Ferrara. Università di Ferrara, 1988. Sez. III; Discussion paper 1 . 
[Mon88b] Marco Mondadori. Classical analytical deduction, part ii. In $A n$ nali dell'Università de Ferrara. Università di Ferrara, 1988. Sez. III; Discussion paper 5 .

[Poe93] Edgar Allan Poe. Edgar Allan Poe: Sixty-Seven Tales. Gramercy Books, New York, 1993. Inicialmente publicado sob o título Works of Edgar Allan Poe.

[Pri62] Arthur Norman Prior. Formal Logic. Oxford University Press, second edition, 1962.

[SC95] Marco Schaerf and Marco Cadoli. Tractable reasoning via approximation. Artificial Intelligence, 74(2):249-310, 1995.

[Sch67] Joseph Robert Schoenfield. Mathematical Logic. The Addison-Wesley series in logic. Addison-Wesley Publishing Company, 1967. Reimpresso pela Association for Symbolic Logic em 2000.

[SK91] Bart Selman and Henry Kautz. Knowledge compilation using Horn approximations. In AAAI-91-Proceedings of the Ninth National Conference on Artificial Intelligence, pages 904-909, 1991.

[Smu68] Raymond M. Smullyan. First-Order Logic. Springer-Verlag, 1968. 